\title{
Metatextual Print Fiction in the Age of Digital
}

\section{Fiction}

\author{
By \\ Mehdy Sedaghat Payam
}

\begin{abstract}
A thesis
submitted to the Victoria University of Wellington in fulfillment of the requirements for the degree of Doctor of Philosophy
\end{abstract}

Victoria University of Wellington 2014 


\section{Table of Contents}

Acknowledgements

Abstract 6

$\begin{array}{ll}\text { Introduction } & 7\end{array}$

Chapter One: Experimental Print Fiction Before Hypertext 22

Chapter Two: Hypertext Fiction and the Remediation of

Experimental Print Fiction $\quad 64$

Chapter Three: From Graphic Surface to Graphical User Interface, Metatextuality in Works of Web-fiction

Chapter Four: Metatextual Print Fiction in the Age of Digital

Fiction

Conclusion 225

Appendix 226

Bibliography 


\section{List of Figures}

Figure 2.1. Storyspace Map View

Figure 3.1. A screen grab of the GUI for Chemical Landscapes

Digital Tales

129

Figure $3.2-3.5$ Three stages of the appearance and disappearance of the text on the screen in Chemical Landscapes Digital Tales $128-131$

Figure 3.6. Interface of 10:01

Figure 4.1. Ground Air Emergency Codes 


\section{Acknowledgements}

There are so many people to whom I am indebted for the completion of this thesis. First of all, my supervisor Brian Opie whose insightful guidance, support, and wisdom was my guiding light during the last four years.

Then I need to thank my family who supported me emotionally and financially during the time I was studying abroad. They were an unending source of motivation in the rainy days.

Last but not least I should thank my friends, for their support, kindness and invaluable conversations which we had. Without your help and support, this thesis would have never seen the light of the day. 


\begin{abstract}
This thesis argues that the future envisaged for the novel by the early hypertext theorists, that the digital medium would displace print and open up a variety of new possibilities for novelistic fiction, can now be differently understood by exploring the materiality of the medium in works of print, hypertext and web-fiction composed in the past fifty years. Michael Kaufmann's analysis of modernist experimental print fiction in his book Textual Bodies: Modernism, Postmodernism, and Print and his use of the term 'metatextual' to locate the distinguishing feature of novels in this tradition will be extended to the works of hypertext and web-fiction in the new media, demonstrating that works of hypertext and web-fiction can be regarded as continuous with experimental print fiction. This analysis, which is also grounded on the concepts of the graphic surface and the materiality of the text, is further confirmed by considering the use of metatextual features of works composed in digital media in experimental novels published in the digital era which continue the tradition by publishing in print.
\end{abstract}

There are four chapters in this thesis. In the first one, metatextuality of the print novels in the pre-digital era is explored through the theory and practice of William Gass who has insisted on the materiality of language and the medium in almost all of his theoretical works. Moreover, the first chapter establishes a point of reference for the discussion of the shift from print to digital media in novel writing by discussing an experimental print novel, William Gass's Willie Masters' Lonesome Wife. Each of the following chapters identify significant issues in the development of hypertext and print for the phase investigated in the chapter, and present two or more case studies of specific texts.

The second chapter explores the development of the novel through the electronic textuality of the early computers. This chapter analyses the first hypertext novel, afternoon, written by Michael Joyce, and how and in what ways it took advantage of the capabilities of the computer and in what ways it tried to remediate print. In order to show how the print novel has been becoming more media-conscious, the second chapter ends with an analysis of a print novel, Fax Messages From a Near Future by Jorge Wilheim which highlights the role of medium in its narrative.

The third chapter follows the line of argument of the previous chapters by exploring the relationship of the multimedia capabilities of the World Wide Web and analyzing the trends which appear through the way the Internet has been used to write novels. The case study section of this chapter includes two novels; 10.01 by Lance Olsen, and Chemical Landscapes Digital Tales by Edward Falco.

The final chapter brings the whole line of inquiry back into print in order to examine what effects the arrival of digital media has had on experimental print fiction and how these novels push the boundaries of the print medium even further. There are three novels in the case study of this chapter, each of which provides a unique insight into the potentials of print and how they bring the materiality of the print to the foreground. The Forgetting Room by Nick Bantock makes the book a multimodal work of art by incorporating the painting and the words. Mark Z. Danielewski's The Fifty Year Sword and House of Leaves make us see the book as a physical object which can be read in a variety of different ways. 


\section{Introduction: Metatextuality and Other Concepts}

In the early phase of the introduction of personal and networked computing, it was widely supposed that print fiction/literature would be displaced by hypertext fiction/literature as a consequence of the shift from one dominant medium, print, to a new dominant medium, computing. Experimental print fiction at times had experimented with the way the materiality of its own medium can be incorporated as within the narrative of the novel. But before arrival of the digital media, the range of these experiments was more limited and even the authors who had done their best to experiment with a variety of print features, had not continued their experimentation into their next work(s).

The introduction of digital media to the literary scene encouraged a number of experimental novelists - some of whom like Michael Joyce had published novels in print - to try their hand at this medium to create works which were both written on the computer and necessarily had to be read on the screen as well. These writers who were later known as hypertext novelists experimented with the materiality of this new medium, and made its materiality an explicit part of the conception of their novels. Although some conventions of the print medium were discernible in the works of these writers, the arrival of the Internet and the developments in digital media provided a significant opportunity for these writers and the new generation of writers to experiment with new conventions for novel writing in digital media. Moreover, these experimentations with the materiality of the medium encouraged the experimental print novelists of the digital era to experiment more extensively with print as a medium. This thesis will explore how the materiality of the medium, either print or digital is used by experimental novelists from the 1960s to 2000s, and how foregrounding this materiality has altered the conception and practice of fiction.

As will be seen, materiality has been a factor in experimental fiction, but literature is not the only social discipline in which this term provides a new angle of interpretation. Paying attention to the materiality of the objects and the way they affect the end-result has become a legitimate subject of study for a wide variety of fields. Tony Bennett and Patrick Joyce, editors of Material Powers; Cultural Studies, History, and the Material Turn (2010) claim the term "material turn" should be used for the change of focus in a wide variety of social studies. 
What has come to be called the "material turn" involves analyzing the materiality of the objects in relation to the place they occupy in relation to other-human and non-human - agents. In their own words, analyzing materiality means analyzing the "differences they [objects] make in their own right as a consequence of their specific material properties considered relationally" (8). What needs to be stressed here is that materiality by itself does not have agency and it is through its placement within a "network of relations" and dealing with a "human agent" that it is activated.

Analyzing a wide range of socio-economic topics, from the role of the filing system in creating the bureaucracy in Colonial India, to the role of road building in Inca Construction at Macchu Picchu, to the role of museums in Australia in shaping the perception of the Aboriginies, the contributors to Material Powers show how the very materials of construction/creation and their maintenance affect what is produced by/through them. Bennett and Joyce define material turn as

a matter of taking account of the distinctive kinds of effectivity that material objects and processes exert as a consequence of the positions they occupy within specifically configured networks of relations that always include human and non-human actors. (5)

An implication of adopting this approach is that it highlights the materiality of the communication and its role in the "network of relations" of social science. When it comes to literature, the definition of Joyce and Bennett sits quite well with the way materially-foregrounded fiction works. The "specifically configured network of relations" in respect of literature has at least three main components of author, text and, reader. Each novel as a textual object with an in-built/designed reading process has (broadly speaking) at least two networks of relations. The first of these "specifically configured" networks is its internal relations which includes both semiotic aspects (signifying strategies) and the design features (physical resources). This network has been configured by the relations between writer/designer (human actor) and the inscription technologies (non-human actor). It is however inactive unless it is activated through the engagement of the reader. 
This brings us to the second network which is the external relationship between what is created through a reading process between a reader and a novel. This process is unique in the sense that it is different for every reader. Here the reader is the human factor and the physical novel (either print or digital) becomes the non-human factor. Through this engagement the reader activates (or puts into motion) the "effectivity" of this textual object which is communication. As reader-response theory has taught us, the kind of effectivity that the second network generates, can be similar to the one intended by the writer, but it is always uniquely different because every reader creates a different network of relations between herself and the novel she is reading. By making the materiality of their novel a part of this process, experimental novelists encourage and assist this uniqueness, whereas the conventional novels try to control this network and offer one preferred way of reading, which may or may not be followed by the reader. The role of each part in this tripartite communication model - author-text-reader - will be analyzed for each case study under a different heading, which will be discussed later in this introduction. It goes without saying that the network of relations expands beyond this model and a comprehensive study of that would involve all the other elements involved such as market, and social systems, which is beyond the scope of this thesis.

Bennett and Joyce's analysis is broadly based historically and culturally, and fiction is not a primary focus, as it is for this study. For fiction, materiality is a key term for understanding some developments in the period marked by the introduction of digital Information and Communication Technologies (ICT). As we will see later, experimental modernist fiction writers had already used the materiality of print to various purposes and this is specifically the topic that Michael Kaufmann has addressed in his book Textual Bodies, Modernism, Postmodernism, and Print (1994). Kaufmann in that book analyzes the presence of the print medium within four novels and uses the term "metatextual" to categorize these novels. The prefix "meta" which ironically in its original meaning means "after" and "beyond" has been used here to describe the novels which make their materiality a part of their signification process. An advantage of this prefix is that in the context of postmodern fiction, it mirrors the way metafiction authors write novels about writing novels. So what Kaufmann means by this term is texts which are about the text, and foreground their textual condition. 
This term is also more inclusive than metafiction because metafictional novels only "comment on their fictiveness" whereas metatextual novels comment on their whole status as semiotic objects and make their own materiality a part of their fictional identity. Metatextual novels also need to be distinguished from the novels which employ metanarrative comment. In the online Living Handbook of Narratology Metanarration as discussed by Neumann and Nunning (2012) "refers to the narrator's reflections on the act or process of narration" (para.1). In other words, metanarration refers to "self-reflexive utterances which address the act or process of narration" (Nunning cited in Herman, Jahn, and Ryan 304). As we will see later in the differentiation between the story and discourse in the same introduction, it is possible to say that metanarration refers to the "self-reflexive utterances" in narrative discourse, whereas metafiction refers to the fictionality of the story. The main reason that the thesis does not draw heavily on this term is that the main preoccupation of this thesis is with exploring a specific branch of experimental fiction, namely metatextual fiction.

Despite its usefulness, this term is not unique for the concept which it refers to; Kaufmann is quick to identify how at least one other scholar has used it to refer to something quite different: "Carl Malmgren uses the term metatextual also but in a much different manner. His use of the term refers to any comment on the text itself within the text. My use of the term refers only to the text physically showing itself" (109).

Apart from the above meaning, the adjective "metatexual" has been used in the discourse of literary criticism to refer to something which at times might overlap with this meaning that Kaufmann has in mind, but is nevertheless different from it. This meaning appears in Daniel Chandler's Semiotic: The Basics (2002). Chandler's definition of this term is: "explicit or implicit critical commentary of one text on another text" (206). Chandler's definition would not apply to the way this term has been used here either.

An important term that needs to be scrutinized here is the term text itself, because it covers a wider range of meanings. Kaufmann's understanding of "metatextual" as a semiotically rich material object informs this thesis, and the 
meaning which Kaufmann's metatextual has already accepted for the term text is the general name for a particular kind of material object that is discussed in his book and in this thesis. In this sense, the text is an example of the "material objects" that Bennett and Joyce had referred to in their discussion of the material turn. In order to narrow down this meaning further, it should be said that the text here has been used as a semiotic object, not the symbolic or signifying components of a communicative or informational object as some other critics might have used it. This conception of text is highly indebted to N. Katherine Hayles' view that there is no information without instantiation, so the text in the sense that some other critics have used it cannot be said to exist, because their understanding assumes a separate entity for the text, which as we will see in Hayles' discussions, cannot exist outside its medium.

Hayles is a prominent scholar who has consistently written on the "materiality of the medium" and this thesis has heavily borrowed from her theoretical discussions and coinages. Hayles advocates a method of reading called Media-Specific Analysis (MSA) which involves paying particular attention to the materiality of the medium in which the work of fiction is presented. The importance of Hayles' analytical method is that it provides a practical method for thinking about text as a semiotic object, and provides a new perspective to think and write about texts which is consistent with the way Bennett and Joyce explain the "material turn." One of the advantages of Hayles' approach is that it brings the medium to the foreground from the very beginning and can be applied for the analysis of both print and digital novels.

According to Hayles in "Print Is Flat, Code Is Deep: The Importance of Media-Specific Analysis" (2004), MSA is defined as: "A mode of critical attention which recognizes that all texts are instantiated and that the nature of the medium in which they are instantiated matters" (67). This definition of text as a necessarily instantiated semiotic object as Hayles later in the same essay discusses, brings the materiality of the medium to the foreground because it identifies the crucial role that the materiality of the medium plays in the signification and interpretation process. Hayles defines two essential components for the materiality of the medium: "Materiality is reconceptualized as the interplay between a text's physical characteristics and its signifying strategies, a move that entwines instantiation and signification at the outset" (ibid.). The advantage of this definition is that it does not 
assign pre-defined roles to these two aspects and the first step in interpretation is acknowledging the role that "physical resources" and "signifying strategies" play in shaping its materiality. These two aspects have a dynamic relationship and either one might have varying degrees of "effectivity" shaping a text's meaning, but breaking the materiality of the text into these two factors means "ensuring that discussions about the text's 'meaning' will also take into account its physical specificity as well."

So now that the meaning of the term "text" and the advantages and disadvantages of the term that Kaufmann has used for the novels that expose their body, have been discussed, let's get back to Kaufmann and have a closer look at his analysis. In the introduction of his book, Kaufmann explores the relationship of the author/narrator to the audience that he is addressing, and argues at length that not all novels which have experimented with the print medium have done it with the same purpose of exposing their materiality and bringing it to the foreground ${ }^{1}$, discusses the distinctive features of the metatextual novels:

[These novels] flaunt their bodies and invite the stares of the readers. They make it difficult or impossible for the reader to see through them. They show their printed bodies for what they are: print and paper. Though few novels call attention to their printed forms as much as Gass's Willie Master's Lonesome Wife (1968), a great many works of the twentieth century disrupt, to one degree or another, normal print conventions. Such works are metatextual: they "show" themselves and comment physically on their material existence in the way that metafictional works comment on their fictiveness. Metatextual works break up the print rectangle of the page and make the physical form of the book "visible" to expose print conventions and the effect of print on language. Typography, footnoting, paginal arrangement, and chapter organizations, all take on different visual configurations. The physical form of the work becomes part of the narrative, so that finally

\footnotetext{
${ }^{1}$ Probably the most well-known example of these novels is The Life and Opinions of Tristram Shandy, Gentleman (1759/1983) which has been cited by many critics as the exemplary instance of a novel which experiments with the print medium (Douglas, 2001, 22), but in Kaufmann's analysis the purpose of Tristram Shandy's metatextuality is different because it "invites the reader to imagine a world on the other side of the page" (33).
} 
the narrative occurs not on the "other side" of the page but directly in front of the readers' eyes on the surface of the page itself. (15) (emphasis mine)

Though Kaufmann does not specifically use the term "materiality" in this definition, his phrase "material existence" refers to the same concept which has been discussed from the beginning of this introduction. As can be seen from the above quotation, he identifies two main characteristics for the experimental novels, according to Kaufmann these novels:

1. Expose their physical body for what it is: i.e. print and paper

2. Disrupt print conventions

The reason that Kaufmann focuses on the works of "experimental modernists" is that in his work he tracks down the relationship between the novelists and their medium - "novel and its use of physical form" in Kaufmann's terms-from Defoe to the modernist writers. According to Kaufmann pre-modern novelists in one way or another have avoided the way print medium affects recording the voice of the writer by either adapting the written communication systems (such as letter-writing and diary) into their works, or treating the print medium as transparent.

Exploring the language-medium crisis, he identifies a trend which distinguishes between spoken language and literary language in the early modernist era. Although this distinction sparks two different reactions in the early modernists, none of them seeks to abandon the meaning beyond the page, for what is on the page. The first reaction was from those who favored spoken language and tried to minimalize the "deadening effect of print" on speech. The second group favored "new experimental language" and wanted to treat the words as "things." This was something that "the printed words-already objects themselves-made easy." By treating words as objects, the second group half-heartedly joins the metatextual camp, because the invisible medium which the conventional arrangement promoted has at least one highly visible component here: words. But this is also where the similarity ends, because despite their fundamentally different approach to language and print, none of them seeks to abandon the meaning beyond the page, for what is on the page and that is why they both look the same on the page: 
Both assume a transparency of print. One attempts to reproduce the voice and the other attempts to use words as things. Though the latter might seem a metatextual approach, readers are not invited to see the printed words themselves but the objects they represent. (Kaufmann 31)

It is only in what Kaufmann calls "high modernism" that some writers such as James Joyce and William Faulkner "used metatextual effects to make print and its effect on language 'visible' by exposing the conventions of print" (32). In this quotation Kaufmann by putting the term visible in inverted commas is signaling how these works were abandoning transparency for visibility. The importance of metatextual works according to Kaufmann is that they highlight important questions about the language and print. Questions like: "what happens to language when it becomes print? Who controls it? Who orders it?" and by doing that show how the medium can play an important role in the narrative, the medium which was transparent and had been designed to erase itself in conventional fiction, in metatextual works becomes visible and asks the reader not to go beyond it. By exposing those traditions, metatextual novels show how the "conventional arrangement might not be an arrangement that suits all fiction equally well" (107).

Kaufmann's observation and the meaning that he assigns to the term "metatextual" become very useful here, because it creates a genealogy for the novels which this thesis is analyzing. From this perspective, Kaufmann's work defines the theoretical background which begins from 1910s to the late 1960s. This thesis, on the other hand, takes the baton from Kaufmann and continues the same line of inquiry from 1960s to 2000s. The media change in the literary scene makes the metatextual trend that Kaufmann had identified for experimental modernist print novels, much more pronounced.

The term "experimental" covers a wide range of meanings when it comes to literature and fiction. The Routledge Companion to Experimental Literature (2012) addresses the same issue:

Experimental literature...is irreducibly diverse. Unfettered improvisation and the rigorous application of rules, accidental 
composition and hyper-rational design, free invention and obsessively faithful duplication, extreme conceptualism and extreme materiality, multimediality and media-specificity, being "born digital" and being hand-made - all of these, and many others, are ways of being experimental in literature. (Bray, Gibbons, and McHale 1)

This broad range of meanings includes the characteristics that Kaufmann cites for a work to be metatextual. Through "unfettered improvisation and the rigorous application of rules" a metatextual work disrupts the print conventions and by "hyper-rational design, extreme materiality and media-specificity and multimodality the metatextual novel exposes its physical body for the print and paper that it is. So in order to be more precise about the terms here, what is meant by experimental novels are the novels which use all of these features in different combinations for example, design, employment of multimedia/multimodal forms, and the use of devices specific to a particular medium.

Sharing the same interest in the implications of foregrounding a text's materiality, but adopting a different perspective, Glyn White uses the phrase "graphic surface" as a means of approaching critically novels which "flaunt their bodies." As the name of his book, Reading The Graphic Surface: The Presence of the Book in Prose Fiction (2005), implies he focuses on one specific aspect of the text's body, that is the ability of the page of a book to act as a screen or display surface. White starts his discussion by stating the obvious fact that each page of any novel is different from the other pages of the same novel, and the horizontal arrangement of the letters, words, and sentences, along with the vertical arrangement of paragraphs "determine" the graphic surface of each page. This two-dimensional space which according to White is "perceived as neutral" in conventional fiction, can be altered by writers who are conscious of the visual aspects of the graphic surface and through "graphic devices." He defines graphic devices as:

An intentional alteration or disruption of the conventional layout of the page of a text which adds another layer of meaning. Some devices may be comparable to the paralinguistic features of speech: pause, volume, hesitation, etc. Others may be visually iconic, forming images which 
directly illustrate or conceptually support the prose. Some devices offer facsimile of embedded texts, while some are mimetic in less direct ways. Others may reorganize the page simply to convey information in more concise forms. In all cases where graphic devices appear they are noticeable because they alter the conventional form of the text which usually is perceived as neutral. (6)

So as can be seen from White's definition, he too stresses the importance of the medium, and through his terminology emphasizes the role of the page as a graphic unit, which is resurrected from its invisible and unimportant status in conventional fiction. Before analyzing White's definition further, it is necessary to make a clear distinction between what is the conventional fiction and why this thesis claims that the page has become invisible in this category of fiction. Conventional fiction as its name implies is a kind of fiction which resorts to the already-accepted convention to present its narrative. As soon as the printing press provided an opportunity for the publishers to mass-produce the novels in book format, they had to make some choices of what goes where and how should a page of a novel look like, what font should it have, and what font size is more suitable. Though the reasons for those kind of decisions is beyond the scope of this thesis, it is worth noting that those decisions have been made according to a variety of aesthetic and economic reasons. However, the rationale behind those decisions has been creating a layout for the page which does not draw attention and eases the reader's move from the words on the page to the world beyond the page. Consequently the page has been pushed to the background and lost its importance in conventional fiction. Experimental fiction, and metetaxtual fiction in particular aims to bring back the importance of each single element of the page and here is where it stands in sharp contrast to the conventional fiction.

Using the graphic devices described above helps to create a graphic surface on the page which in White's definition is:

a free two dimensional space on which text appears either mechanically (as a result of the translation of the manuscript into print) or consciously (when the author wishes to adapt it for specific purposes). As visual arrangements of printed text on the graphic surface, graphic devices can contribute to the process of reading, 
combining with the semantic content within the context which that text creates. The graphic surface thus allows authors a second mode of signification, one which may point out the constraints of normal printed language, but which may simultaneously supplement that language and contribute to the generation of meaning. (206) (italics mine)

Graphic surface in this sense opens up a dynamic relationship with the signifying strategies of the text which results in the emergence of the meaning of the text through this "simultaneous supplementation". This simultaneous supplementation is an important phrase here because it shows the inter-relation of the two modes of signification which are simultaneously at work in a novel, its signifying strategies and physical resources. These two modes come together in the different parts of author-text-reader relation as we will see in the tripartite structure which has been used for studying each case study here.

What is particularly useful about the term graphic surface is that it addresses two issues which are fundamental to the understanding of the materiality of the medium. The first one is "graphic" which according to Merriam Webster dictionary refers to a wide range of meanings, almost all of which are related to the materiality of the medium in fiction, therefore, talking about graphic surface in fiction, apart from its design implications which will be discussed later, highlights the page as a surface "formed by writing or drawing" and presented by "the art of printing", which has a visual [and sometimes "pictorial"] element, aims to "convey meaning" through "printed word", and sometimes tries to represent "vivid realistic description." This term draws attention to the visual elements of the page and their graphical organization that defamiliarizes the page of the print novel. Another advantage of the word "graphic" is that it can also be used for hypertext and web-fiction and this is one of the reasons that makes this term useful for the novels which are analyzed here.

The second word in this phrase is a direct result of foregrounding the graphic characteristics of the page, when the word and the arrangement of the words on it become important and stop being invisible, the page itself becomes the location of intersecting the physical resources and signifying strategies which according to White leads to the formation of an additional mode for interpretation. Using the term 
additional, White intends to say that there is already one mode of meaning in the text which has been created by the words alone and tinkering with the materiality of the medium creates a second additional mode.

A third aspect of the implications of White's term is highlighting the role of design as a prominent feature in works of experimental fiction. One of the common collocation for the word graphic is "design." When the physical resources of the medium - either print or digital — become visible and play an important role as the second mode of signification which has a simultaneous supplementation relationship with the signifying strategies, their arrangement and the way they come together in the design of the book becomes critically important. In other words, the graphic designer-whose name may or even may not simply appears along the other copyright information of the book-becomes the co-author of the novel. The designer's role become more complex in the works published in digital media, and though what the designer has done might be overshadowed by the directions that the writer has given him/her in print (House of Leaves (2000) and Fifty Year Sword (2005)), he has a more outstanding role in works like digital version of 10:01 (2006). Hence, the role of designer has been acknowledged and analyzed in the case studies of this thesis, and wherever the confluence of the designer and author has resulted in a tension between the two (as is the case in Willie Master's Lonesome Wife), the role of each and the way this confluence has affected the final product has been discussed and analyzed.

White's discussion of modes overlaps with the way Alison Gibbons explores the experimental literature in her book Multimodality, Cognition, and Experimental Literature (2012). ${ }^{2}$ Similar to Kaufmann and White, Gibbons is dissatisfied with the way the material properties of the novels have been neglected by the literary criticism, and how an already established critical commentary for experimental novels does not exist yet. "Inevitably, literary criticism has instead favored the word, and often not as the written word, focusing instead upon the thematic and conceptual facilities of discourse" (1) (emphasis in the original).

\footnotetext{
2 Interestingly enough she starts her introduction with "novels that feature graphic elements..." (emphasis mine), which links the object of her study with the way White had approached these experimental novels. She also quotes White in the first paragraph of her introduction.
} 
Gibbons' own book and her terms for this group of experimental novels, "multimodal" novels are aimed at filling this gap. Broadly speaking, the theoretical discussions of her book can be divided into two sections: 1.multimodality in contemporary literature, 2.the way this multimodality affects cognition. In her own words she aims at putting "multimodal cognitive poetics into practice through analysis of literary texts". Since Gibbons' work has been discussed more fully in the last chapter of this thesis, a short explanation of the overall scope of her thesis is presented here.

Both multimodality and cognitive studies are new fields of study which have not been fully developed yet. As Gibbons' literature review suggests, there are a number of scholars who are still theorizing different aspects of multimodality, but there seems to be less controversy regarding the definition of multimodality which according to Gibbons in its most fundamental sense "is the coexistence of more than one semiotic mode within a given context." The key term in this definition is "mode" and understanding what multimodality is depends on what is counted as mode. Gibbons takes Ruth Page's definition of the mode and quotes that in her book.

The modes described in multimodal analysis refer specifically to semiotic modes (as opposed to other, specialist uses of the term). Thus a mode is understood here as a system of choices used to communicate meaning. What might count as a mode is an open-ended set, ranging across a number of systems including but not limited to language, image, color, typography, music, voice quality, dress, gesture, spatial resources, perfume, and cuisine. (Gibbons 10)

The importance of this definition for literature is that it places language and its material manifestations (such as typography) side by side and does not prioritize one over the other. What it means for fiction as a semiotic construct is that the materiality of the manifestations of language (the kind of typography chosen for the words, their color, and even the size of the fonts) become equally important as the words and language because they are all modes of communication. What the metatextual novels do, and the way they foreground their graphic surface inevitably brings in other modes of communication (more specifically color and typography, as we will see in chapter one, and four for print novels and in chapter three for works of web-fiction), 
and by doing that undermine the dominance of one specific mode as the "preferred mode" of communication.

Since the novel as a specific genre and media form developed its defining characteristics and conventions in association with the evolution of print technology, the question of how narrative is transformed through works of hypertext or web-fiction is a significant one. Because experimental novels in the print era have foregrounded ways in which aspects of print technology have been instrumental in their composition, the experimental novel is being taken as the point of reference for considering this question. H. Porter Abbott has a useful definition of narrative and his definition will be used as a guide in the controversial subject of narrative and how it should be thought of in the works of hypertext and web-fiction. In Abbott's definition, "narrative is the representation of events, consisting of story and narrative discourse." Story "is an event or sequence of events (the action), and narrative discourse is those events as represented" (16). The main reason that Abbott's definition has been chosen here is that it can be applied to the study of narrative in an almost any medium; Abbott has also devoted several pages of his book on narrative to narrative in hypertext.

Looking at a novel from the modality perspective, provides us a better understanding of how each work is created out of the different combinations of modalities of three different but related categories. A fictional text uses a specific modality of the text which is the narrative genre. It uses the modality of the medium either print or digital. The last modality which comes into play here is the modality of verbal/visual which is part of the modalities of representation. These modalities can work in different combinations, but segregating them in this way, makes them more visible and shows how each writer can create texts, by manipulating either of these so that the reading process would be affected by the way either of these modalities is brought into play.

Thus, in order to study the materiality of the fictional works discussed here, three different but interrelated dimensions of the fictional text have been identified. The first dimension is Physical Organization and Design in which the materiality of the text from the perspective of the use of its physical resources-which in 
Kaufmann's terms would be "textual body"-its authorship, and design has been analyzed. The second dimension is Narrative Strategy, and through this angle the materiality of the text from the perspective of the use of its physical resources and signifying strategies to create a narrative is analyzed. The third angle is the Reading Process, in which as its name states the materiality of the text in the dimension of its shaping the experience of the reader has been explored.

This thesis is divided into four chapters which explore the materiality of the medium in pre-digital, hypertext, web fiction, and digital-era experimental print fiction. Each chapter is divided into two parts, the first part is the theoretical discussion which draws upon critical materials related to each phase of development, and then the chapter moves to a case study for novel(s) which demonstrates the value of thinking about these terms for interpretation of the novels.

Now we can return to the main question of the thesis which is how does the material turn occur in fiction and what happens during almost 30 years which distance the publication of Willie Masters' Lonesome Wife and House of Leaves-a metatextual novel that becomes a best-seller? A comprehensive answer would require a deeper analysis of what technology brings to the table and how the introduction of the digital medium sheds new light and ultimately changes the perception of the books. In other words, this thesis would try to show how this "material turn" has occurred in fiction by analyzing the ways in which materiality of each text demonstrate both an evolutionary process for narrative fiction through the introduction of a new writing-publishing technology after print and how is the conception of narrative fiction modified in the process. 


\section{Chapter One: Experimental Print Fiction Before Hypertext}

"The true alchemists do not change lead into gold; they change the world into words." (Gass, A Temple of Texts 37)

[A separation between art and life] supposes there are parts of life - those that are not symbolic, not mediated by signs, not mediated ever in the general sense-that they are more real than those that are mediated. I don't see how it's possible now to look at the world and not see the whole thing penetrated in both directions. (Gass, qtd. in Kaufmann 98) 


\section{Introduction}

The material turn in fiction depended on foregrounding two elements which as Kaufmann shows in his book, had either been expected to self-erase or behave as transparent components of the work of art. These two elements were the medium in which the fictional work had been instantiated in and the language in which this work had been presented. This chapter will analyze the precursors to digital modes of foregrounding the materiality of the medium in theory and practice of pre-digital writers. The works of high modernists had already paved the way toward a new understanding of fiction, accompanied by breakthroughs in other art disciplines. What is meant by the pre-digital era is the era which immediately precedes the introduction of Computer as inscription technology in the early 1980 (IBM's PC in 1981, and Apple's Macintosh in 1984) (Cerruzi, “A History" 268-276). As McCaffery has observed in his book, Metafictional Muse: The Works of Robert Coover, Donald Barthelme, and William H. Gass (1982), this era in itself can be divided into two parts, the first one from 1945 to 1960s in which "with only few significant exceptions (Barnes, West, Hawkes, Patchen) American fictional approaches remained largely conservative," and the 1960s and 1970s, when the literary climate-assisted by the radicalized social and political atmosphere-rapidly changed. "Virtually overnight, it seemed our admiration for the important writers of the 1950s was transferred to a literary generation of a very different style and temperament indeed: Heller, Barth, Vonnegut, Coover, Barthelme, and Gass (19)."

This new "different style and temperament" in fiction, which McCaffery (and as we will see a variety of other critics) named "metafiction," focused on the role of language — and for some writers, print medium — in its own construction. None of the writers identified by McCaffery have written more extensively and zealously about language in fiction than William Gass. The main reason that Gass has been chosen for this chapter is that in exploring Gass's works we can see that for the purposes of this study, his novel Willie Masters' Lonesome Wife (1968) is a foundational text. Its graphic surface uses different graphic devices to foreground its own physical body and the result of all those graphic devices is a novel which so radically foregrounds its print medium that according to Kaufmann "few novels call attention to their printed forms as much as Gass's Willie Masters' Lonesome Wife"(14). It vividly uses several 
modes (language, photos, and colors) within its physical form, as we will see in the case study, and in this way emphasizes emergence of the text and its meanings by means of a mode of organization (narrative) in interaction with a reader.

\section{Role of the Medium}

A familiar understanding of a work of art is that it is a window to real or imagined real worlds. During the history of English literature, several literary schools have competed with each other, each one claiming to offer a more accurate picture of reality. Novels have always been mediums which lead the reader through them to a world which is the real world or like the real world in its spatial/visual form. These worlds exist beyond the page and the language and materiality of the novel was expected to be effaced during this process.

Moreover, many novelists believed that there are some "pure notions" such as objectivity, absolute truth, etc. and human beings through the empirical data which they receive from their senses, could understand these notions and the "deterministic universe" in which these concepts exist. This view implied that human senses objectively transferred perceptions of the outside world to the subject and the senses while playing their mediating role, did not have any effects on the data or the perception of the data. Therefore, language which has been a system developed to communicate between human beings, was a "pure" means which could transfer the meaning from the author's consciousness to the readers'. Language was also devoid of any effects on what it transferred. In other words, it was just an insignificant vessel which a writer used to pour the concepts and meaning into, and transferred them into the reader. Consequently it is not surprising that the naturalist and realist writers did not take the role of language into account, and believed in a neutral role for it.

However, after the "demolition of the faith in rational, empirical investigation, the frank acknowledgement of the subjective nature of our mental operations and their relationship to the world," (McCaffery 11) the manipulating role of language as the mediator between consciousness and outside world was recognized by writers in the 1960s and 1970s. In the early 1970s, Robert Coover made an important claim that was shared by many writers of the time. According to Coover: "Our old faith ... derived from myths, legends, philosophies, fairy stories, histories, and other fictions ... as lost 
its efficacy" (qtd. in McCaffery 8).These fictions which are all necessarily made out of language are our only means of understanding the world and "what happens to us from day to day (ibid.)." Thus language becomes the only means of cognition and this is why consciousness is embodied in language.

\section{The Invisible Language}

Due to the influence of the above-mentioned theories, these two decades brought a fundamental change in the "fiction-making process." Within a short period of time, the "optimist, empiricist view that assumed the existence of a deterministic universe and the human ability to uncover the most intimate mechanisms of its operations" (McCaffery 9) was replaced by a view which saw the human being as "locked within his own forms, languages, and fictions which he creates and manipulates as useful aids" (ibid., 11). Whereas the former had helped establish the conventions for realist fiction "with its logical, casual connections and linear development," the latter became a common theme for postmodernist fiction. According to McCaffery:

Works of postmodern imagination often reflect this new epistemological orientation quite directly. Thus many new fictions deal openly with the hazards of knowing and with the systems we create to help us navigate through our daily experience. Widely differing structural devices helped reinforce the turn away from realistic norms: the use of multiple contradictory perspectives, Burrough's "cut-out" methods, the increasing willingness of writers to use graphics and typography in their works, the deliberate confounding of fact and fiction, the general tendency of writers to flaunt their artifice....not surprisingly, many of these works focus on the mediating effects which language and literature have on man's relationship to the world....These writers are well aware that consciousness is embodied in a particular language and that it is our language which generates our response to the world. (13) (italics mine)

One of the novelists who took up this task of directly challenging the perception of written language as an invisible medium, and used "graphics and 
typography" in his works and "flaunted their artifice" was William Gass. He also wrote several books of essays "on the mediating effects which language and literature have on man's relationship to the world" and made it a recurrent theme of his novels. Gass's first book of theory and criticism Fiction and Figures of Life (1971) has been hailed by McCaffery as "the most important single work of criticism to appear during the period [of early 1970's]....a study that provided the non-traditionalists with a manifesto which justified their efforts in terms of carefully established literary and philosophic principles" (23).

Gass in that book and several other books which he has published during the last four decades challenges the conventional theory of reading and writing fiction. According to Gass, a work of art's material body is as important as the message or concepts which the work of art is transferring from the writer to the reader. A novel might be about human relations, love, honor, etc. but, it is the codex format of the book, and the written form of language which embodies all these concepts, and although all of these have been designed to facilitate this communication between the writer and the reader, their mediating role should not be ignored. The codex format is the medium through which the message is transferred and this medium as was discussed earlier, directly affects our perception of the message or concepts. This is why Gass says that a true alchemist is a person who changes the world into words. Human consciousness, after all, understands the world through the "fictions" which are created by language, and the words are the smallest meaningful unit of language, thus a true alchemist by changing the world into the words, makes something which is not intelligible to the medium which helps us understand it.

Therefore, it can be concluded that according to the new understanding of fiction in the second phase of pre-digital era a work of art is not an invisible container for the concepts that author pours into it, but it has a unique identity of its own. Furthermore, this identity is materialized through a body which is not supposed to erase itself or become transparent during the reading process. Thus a novel or generally a work of art is not a window to reality, but something which its material form plays an influential part in its identity. The writer has to use a medium in order to transfer his ideas and concepts to his audience, because his ideas are abstract and 
intangible. Hence, a writer has to use the medium of language to make this transference possible

The above-mentioned aspects make Gass's works stand out both in theory and practice, when fiction is regarded through the perspective of the material turn. Despite having published more books of essays than novels, Gass considers himself a fiction writer and in an interview with Hungarian Journal of English and American studies, he has clearly stated that fiction is more important to him and that is what he really wants to write. Gass has proposed an approach to writing fiction in his works which as we will see has major overlaps with the metatextual novels which Kaufmann had described, and all through his theoretical writings, he supported and championed this style. There are few essays in which Gass directly addresses the print or electronic medium, but a keyword in understanding Gass's ideas is the materiality of language.

As was discussed above in the second phase of the pre-digital era the literary climate changed and this change resulted in experimentation and search for new modes of expressions for the writers and novelists. Consequently, during these two decades several new forms of fiction were created which were later categorized under different rubrics such as "surfiction," "aleatory fiction," "parafiction," "super-fiction," "metafiction" etc. Among these new forms, metafiction became the most prominent and widely discussed term in literary circles and academic scholarship. This term which has been coined by Gass has generated some full-length books and several definitions by a variety of critics - McCaffery (1982), Hutcheon, (1988), Waugh (1984), Currie (1995) ${ }^{3}$ — but a recent definition by Joanna Gavins directly addresses the issue of language which is highlighted in this chapter as well. According to Gavins in the Routledge Encyclopaedia of Narrative Theory (2005) "[metafiction] foregrounds the nature of fiction as an artifice that both mirrors and refracts reality such that it reflects back on how the language of narrative achieves the effect of

\footnotetext{
${ }^{3}$ R.M. Berry in Bray, Gibbons and McHale provides a summary of the history and the developments of the term by these critics until 2012. Each of the definitions that these critics have offered focuses on a set of features which according to that writer are the defining aspects of the novels categorized by this term. But the definition chosen here has this advantage over the others that it brings back the language into the mix, and as we shall see this makes it closer to the original context in which Gass had coined this term.
} 
referencing reality through imaginary means" (O’Donnel in Herman, Jahn, and Ryan 301) (emphasis added). With its emphasis on language and "fiction as artifice" this definition becomes closer to the metatexuality which Kaufmann has discussed in his book. So it goes without saying that in order to be metatextual, a work necessarily has to be metafictional because "flaunting the body" is part of the stressing the artificiality of the work of fiction, but whereas a metafictional work may appear in the conventional format of the book and limits its reflection on "referencing reality through imaginary means" to language only, a metatextual work goes one step further and brings its print medium and physical body into the equation as well.

\section{Language and Metafictionality}

As McCaffery shows in his book, language and the legitimacy of its claim to reality had come under attack from different disciplines within science and humanities. In these disciplines a "meta" discourse was being developed to examine how "subjective elements enter into all human operations" and confirm that "perhaps even our sense date were from the outset primarily symbols, the product of a synthesis between matter and subjectively provided form" (9). It is in an effort to bring this line of inquiry to literature that Gass coins the term metafiction:

There are metatheoremes in mathematics and logic, ethics has its linguistic oversoul, everywhere lingos to converse about lingos are being contrived, and the case is no different in the novel. I don't mean merely those drearily predictable pieces about writers who are writing about what they are writing, but those, like some of the work of Borges, Barth, and Flann O'Brien, for example, in which the forms of fiction serve as the material upon which further forms can be imposed. Indeed, many of the so-called antinovels are really metafictions. (Gass, Fiction and Figures 25)

What he is emphasizing in the above quotation-and that goes on to be the central emphasis in works - is the role of language. As Berry has observed, this paragraph comes at the conclusion of an essay in which Gass claims "the art of the novel is now a mature art" (Gass, Fiction and Figures 26), and according to Gass, this maturation is due to the novelist's "ceasing to pretend that his business is to render the 
world; he knows more often now, that his business is to make one" (24). This stance is consistent with the famous quotation from Gass that "there are no descriptions in fiction, there are only constructions" (17), which appears in the same essay. Therefore, when the writer has to make a world rather than to describe/represent one, he has to pay particular attention to the materials of this construction which are written language and the medium in which this language is instantiated. In a later

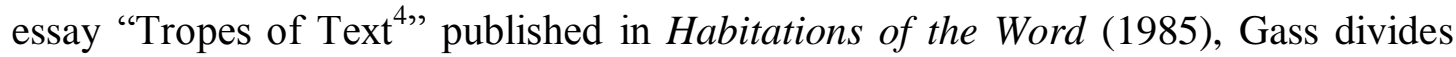
authors according to the way they have treated these two media into three groups: preliterate, literate, and transliterate. He uses the examples of three novelists to demonstrate why these categories are important. The first one, Theodore Dreiser,

is a preliterate novelist $[\ldots \mathrm{He}]$ wrote as if words had little resonance, no sound, no shape, as if their inscription were invisible, and he employed a syntax as uncomplicated and casual as a wad of cotton.... and indeed we do soon wish to pass through his words to the world he is asking us to imagine. (150)

For Dreiser, language is transparent because he does not aim to construct a world, he wants the imagined world of his novels to seem completely realistic, hence, he does not particularly attend to language and the print medium in which his novels appear. This is the main reason that he pays no attention to the language or print and is asking the reader to "pass through his words to the world he is asking us to imagine." In other words, Dreiser had only one aim in using language and print medium, which is the aim of realism - to use them only for referential function. To go further was to recognize that language as a system of signs does much more than refer, and that it can be used artistically like any other medium of representation. As we will see in the next paragraph, this is what James does but doesn't slip into the opposite error to Dreiser's, which is to give full value to the artistic side of language and put the referential to one side.

Henry James is literate. James "feels a larger obligation, and plumps his page like a pillow, for he is literate to a fault like the golden bowl's" (ibid.). According to

\footnotetext{
${ }^{4}$ This phrase is more popular with Gass because it emphasizes the text, rather than the figures of speech which emphasizes the speech not the text.
} 
Gass, Henry James pays utmost attention to the construction of every individual sentence and he always creates the best possible combination of words in his fiction. In this sense he becomes like a goldsmith who creates a golden bowl through the raw material of gold. This emphasis on construction rather than description/representation for language in fiction makes him literate.

The third category in Gass' classification is the transliterate author. Such a writer in addition to attending to the language pays particular attention to the medium in which his linguistic work is instantiated, and this makes him a metatextual novelist in Kaufmann's terms.

Joyce worries about the color of the cover for Ulysses, since he is transliterate. And after all, if alliteration is important, why not the relation of closed spaces to open ones in a type face? Why should the long sounds in short words astonish? Why should the implications of one materiality lie idle while another is worked and worn out? (ibid.)

In other words, the transliterate author pays attentions to two materialities; the first one is his language and he "plumps his page like a pillow," the second materiality for him is the materiality of the medium in which his work appears, and since Gass is talking about James Joyce who published his novels in print medium, the features of this medium which are taken for granted by the other two groups, are brought to the foreground as well. In other words, a transliterate author creates an interaction between the physical resources of his novel and its signifying strategies.

Kaufmann only devotes one line to discussions of Gass's transliteracy and in brackets right after this term explains: "his [Gass's] word for using all aspects of print." Since Kaufmann has been discussing the print novels in his book, this short interpretation of Gass's transliteracy is suitable for that specific context, but it is inadequate for describing novels in other media. Though Gass's term "transliterate" is a useful term here, its emphasis is on the attributes of the author's conception of his work and it is mainly concerned with the ways in which "the letter" is either a fact, or not, in the work of the text in establishing a communicative relation with a reader. In this context, 'literate' means conscious of the distinctive attributes of the letter which appears in the written form of the language) and transliterate means what includes but 
goes beyond the letter which are all the other non-linguistic attributes of the medium. This thesis takes Gass's term and reframes it to underline the point that it's the text which combines/instantiates these attributes and therefore it is texts, not authors, that are the focus of this thesis.

\section{Gass's Metaphors}

In every art two contradictory impulses are in a state of Manichean war: the impulse to communicate and so to treat the medium of communication as a means, and the impulse to make an artifact out of the materials the medium and so to treat the medium as an end. Calligraphy is an obvious instance. The elaboration that can be accorded the letter $\mathrm{R}$, for example, far outruns its meaning, yet it would receive no elaboration at all if it were not a letter. One is tempted, therefore to see in the elaboration some explication of the meaning of the letter, some search for mystic essence even, while at the same time the elaborations reduce it to a pure design whose interest lies wholly in the movement and harmony of lines in space (Gass, Willie Masters’ Lonesome Wife TS n.pag)

What Gass means by transliteracy, is further elaborated in his critical essays where Gass according to McCaffery, rather than 'gloss, explicate, 'argue' in the traditional sense," uses "striking similes, and elaborate analogies and metaphors" (qtd. in Hix 56) to explain his idea. Therefore, in the following sections, some of these metaphors and similes which Gass has used in his essays for the art object—sculpture, a chair, a beautiful woman, and music-will be analyzed. These metaphors, in addition to providing a better opportunity to explicate "transliteracy" and shedding further light on what he means by the materiality of the language, indicate how language is to be materially understood. The common theme behind these metaphors is Gass's idea about construction of a work of art and how the materials are used to create a new whole which is a new object. Also, as can be seen from the above quotation, Gass believes there are two impulses in the work of art "the impulse to communicate and so to treat the medium of communication as a means, and the impulse to make an artifact out of the materials of the medium and so to treat the 
medium as an end." These two impulses are the result of the emphasis on the same two categories which Hayles later identifies as signifying strategies and physical resources. Too much emphasis on the signifying strategies of a work of art would make its medium a transparent means for communication. This is what Dreiser does, because his strategy is to reduce what is distinctive about language as a medium to zero. He treats the language as means for communication and the novel for him is nothing other than signifying strategies. On the other hand too much emphasis on the physical resources would result in the medium being treated as an end in itself and undermines the signifying strategies. A good example for the second part, which Gass also points out, is the way language can be used in calligraphy in a meaningless way, just for its visual characteristics. A good work of fiction does not fall into either of these extremes, in fact, a good writer is the writer who creates a balance between these two in a way which neither attracts too much attention to itself.

\section{Sculpture}

On the other side of a novel lies the void. Think for instance, of a striding statue; imagine the purposeful inclination of the torso, the alert and penetrating gaze of the head and its eyes, the outstretched arm and pointing finger; everything would appear to direct us toward some goal in front of it. Yet your eye travels only to the finger's end, and not beyond. Though pointing, the finger bids us stay instead, and we journey slowly back along the tension of the arm. In our hearts we know what actually surrounds the statue. The same surrounds every work of art: empty space and silence. (Gass, Fiction and Figures 49).

In the first half of this quotation, Gass describes the visual aspects of the statue and identifies its signifying strategies. All the adjectives here-purposeful, alert, penetrating, outstretched, pointing ${ }^{5}$ — describe a statue in a purposefully moving pose. However, the word "yet" in the beginning of the second section directs the readers" attention toward something different. Although the statue is in the walking pose and is

\footnotetext{
5 These adjectives are not inherent for the statue, but they are actually an expression of the consciousness of the viewer connecting with the consciousness of the author/artwork. If the they were "in" the statue, then there would be only one meaning for the statue/work of art and the issue of different [and sometimes conflicting] interpretations would be totally out of the question
} 
pointing toward something, it is actually static and our eyes instead of looking at the direction that the statue is moving to, look back toward the body of the statue after we reach the pointing finger which as Gass aptly observes "though pointing, the finger bids us stay". The reason that our eyes "journey slowly back along the tension of the arm" is that a statue is not a directional sign and neither it is going somewhere. Neither its sculptor nor the sculpture itself, want the viewers to follow the pointed finger. This foregrounds an important issue here; the materiality of the statue and the way it is presented to the viewers; instead of trying to efface its existence to lead the audience to something beyond it, asks the audience to stay and appreciate it for what it is and what it says, rather than trying to regard it as a sign which asks the viewers to move toward the direction that it is pointing to. In other words, although the statue is pointing toward something, this pointing is part of the overall meaning of the statue and has not been meant to serve a directional role.

The statue conveys its meaning through the visual form (as opposed to verbal), but the comparison that Gass is making here with the book is because of the materiality of both as artifacts. Since language in other disciplines is used as a transparent medium, if we treat the language of work of fiction in the same way that language is being used in its daily use, we will be like the viewers of the statue who sees the pointing finger and looks for the meaning that the statue is trying to communicate at where the statue seems to go or where its finger is pointing to. Moreover, a transliterate writer does not stop here and pays attention to the smallest details in the physical resources of his work, so that the signifying strategies of his work would complement and compliment these physical resources and the verbal equivalent of the statue that he has made asks its readers not to go beyond it, but stay and appreciate its beauty both at the level of language and at the level of its physical body.

\section{Chair}

"It seems a country-headed thing to say, that stories and the places and the people in them are merely made of words as chairs are made of smoothed sticks and sometimes of cloth or metal tubes" (Gass, Fiction and Figures 27). 
In this metaphor, Gass reminds us how similar stories and chairs are. Chairs are made out of materials that are available to almost all the people. Sticks, cloth, or metal tubes can be easily obtained from the market if someone wants to build a chair, in this sense they are similar to words that are available to everyone, but it is not the material by itself, but the work done on them which creates a totally new object out of them. In other words it is the transformation of the ordinary by the application of art which is conscious use of skill and creative imagination here.

Similarly stories are made out of words, which are available to everyone, but when through the "conscious use of skill and creative imagination" of the artist — writer - they are made into an artifact $^{6}$, which is a work of fiction here, they become a new aesthetic object. Whatever the common use of the sticks, cloth, or tubes has been, the new object which is made out of them changes that role and makes them components of a new object with a new identity. Thus, language in a fictional text is not the same as language in its day-to-day use, because through art it has been transformed to a new object which is a work of fiction. However, the utilitarian function of the language has cast such a big shadow over the language in fiction that "saying stories are made of words" and do not serve a representational role, seems to be a foolish thing to say. The direct result of this blindness toward the transformation of words in fiction is taking the materiality of the book for granted while reading a novel. In the same way that we take a chair's materiality into account we should take the materiality of novels into account as well.

\section{Beautiful Woman and Slaves}

The moment our writer concentrates on sound, the moment he formalizes his sentences, the moment he puts in a figure of speech or turns a phrase, shifts a tense or alters tone, the moment he carries description, or any account, beyond need, he begins to turn his reader's interest away from the world which lies among his words like a

\footnotetext{
${ }^{6}$ In fact the word artifact in itself is very interesting here. According to OED, an artifact is derived "from Latin arte 'by or using art' + factum 'something made." Thus an artifact is an object made by or using art, and its contemporary meaning "an object made by a human being, typically one of cultural or historical interest" still signifies both the object status and the human maker of it. It can be said that in this respect, an artifact shares these two attributes with the chair and a work of art.
} 
beautiful woman among her slaves, and directs him toward the slaves themselves. (Gass, Fiction and Figures 32)

The main similarity here is between a beautiful woman and a finished work of art. The words are like the slaves which help to make an aesthetic object. Although they are the building units of that object, their role in the final design of that object should be in such a way that the audience pays attention to the finished work rather than the materials which have been used to create it. This stance might seem to be in contrast with Gass's previous ideas about foregrounding the materiality of the text, but a closer inspection of Gass's ideas reveals that Gass is always stressing the importance of the materiality of the finished objects, not the material used for its creation. In the previous metaphor, we saw how the materials used to create an artifact found a new identity, not as single individual parts, but as the parts of a new whole. Here Gass goes one step further and discusses that in creating this new object, the individual elements should be put together in a way that do not draw attention to themselves and merely serve the purpose for which they have been brought into this artifact. When it comes to fiction all the elements in creating a novel should be combined artistically to produce the desired effect and none of these should stand out in a way that diverts the attention from the overall work to itself as an individual component of that work. For instance when Gass talks about the statue, he never mentions whether the statue is made out of marble, bronze, or any other material, because the materials used for creating the statue are just like the slaves, if used successfully, would not draw attention to themselves, but help the audience to see the beauty of the work of art.

\section{Music}

"You do not tell a story; your fiction will do that when your fiction is finished. What you make is music, and because your sounds are carriers of concepts, you make conceptual music, too." (Gass, Fiction and Figures 274)

Gass in this metaphor emphasizes the similarity between music and stories. Music is created through individual notes, which alone do not convey any meanings, but when they come together they form a piece which is more than its material parts. Different modes of music can evoke various feelings without the use of concepts. 
However the words, which Gass refers to here by their aural attribute, have sound as well as concept. So the reader has to be as meticulous as a composer in arranging the words together to create a work of fiction which serves his purpose in the best possible way.

The words and the language have an aural dimension to them too, which is largely ignored while reading a story. The conceptual music, which communicates meaning through the notes to the listener, does not seek to efface its materiality for the reader, and in the end it still remains music. It might seem that Gass's ideas are the same as the aesthetics of Art for Art's Sake, but that is not the case. Gass might not believe in a moral or didactic function for art, but for him a work of art and specifically a literary work has a purpose, according to Gass: "The purpose of a literary work is the capture of consciousness and the consequent creation, in you, of an imagined sensibility, so that while you read you are that patient pool or cataract of concepts which the author has constructed" (ibid. 33).

So the audience here are not the observers who are watching a representation of the real world through the window which a work of fiction has created. Instead they are similar to the audience of a piece of music, which does not represent anything but creates a "cataract of concepts" in the listener. This issue will be further investigated at the reading process of Willie Masters' Lonesome Wife.

\section{Language in Fiction}

These metaphors together show how Gass thinks about the modality of the fictional text in the context of art objects in general. Understanding the points that he makes by each metaphor has a critical role in interpreting Willie Masters' Lonesome Wife as an example of an effort in metatexuality and transliteracy. What these metaphors reveal about Gass's conception of the artwork in different modalities is that the artwork does not have a referential role and because it is a new artifact, its materiality is an important feature in understanding its communicative purpose. On the other hand, an artifact is the result of the transformations of each individual component into a different whole and assigns new meanings to that. As for fiction, what remains particular to/predominant in it is the language and this is the reason that 
throughout his oeuvre, Gass emphasizes how language in a work of fiction should be, and how different it should be from its utilitarian use in everyday life.

Although Gass' works is experimental in various ways, we should bear in mind that Gass is totally against experimentation for the sake of experimentation and in the foreword to Transgressions, the Iowa Anthology of Innovative Fiction (1994), he says:

What is generally called "experimentation" in the arts more nearly resembles my ignorant and useful self-indulgent mess-making [in my chemistry lab]. I was acting out a fantasy, not learning anything about chemistry, and while every smelly substance I concocted had to have been made according to chemistry's laws, I did not know those laws, nor could I have learned them from anything I was doing. We have to imagine an artist wondering what would happen if she were to do this, try that, perform a play in silence, omit the letter " $E$ " in three pages of French prose, construct a world of clothes hanger wire, color walls with cow manure. Having found out, though, then what?" (3)

The art object materials which help the writer to create his novel, are words. For a writer, words are slaves in the way that they work together to create a beautiful woman, that is a work of art. Since people use language for utilitarian purposes, it would be much more difficult to expect them to change their outlook toward language while reading a work of fiction which is created by the same words that they use for purposes other than creating an aesthetic object. According to Gass, this is also one of the reasons that the medium of language has been treated as transparent by the novelists of the past and present. In other words, one of the major problems for novelists is that the material by which they make their artifacts is not exclusively used for their creation, in the way that the palette or the music notes are exclusively available for the painter and the musician. A composer or a painter's material is something that almost nobody except the painter or the composer would use during the everyday life:

The composer's medium is pure; that is, the tones he uses exist for music and are made by the instruments especially designed. Imagine 
his feelings, then, if he were forced to employ the meaningful noises of every day: bird calls, sirens, screams, alarm bells, whistles, ticks, and human chatter. He could plead all he liked that his music was pure, but we would know that he'd written down sounds from a play unseen, and we would insist that it told a story. Critics would describe the characters (one wears a goatee) and quarrel over their motives, marriages, or mothers, all their dark genes. (Gass, Fiction and Figures 30)

But language, the main medium of novelist, is worn out because of overuse:

The novelist makes his book from boards which say LADIES and GENTS. Every scrap has been worn, every item handled; most of the pieces are dented and split. The writer may choose to be heroic...he may strive to purify his diction and achieve an exclusively literary language. He may pretend that every syllable that he speaks hasn't been spit, sometime, in someone else's mouth...Most novelists, however, try to turn the tattering to account-incorporate it cleverly-as the painter does when he pastes up a collage of newspaper, tin foil, and postage stamps. (ibid.)

These quotations make it more clear that for Gass, transparency of language in its everyday use makes it more liable to being treated as serving a referential role in fiction as well. After all, seeing through language makes it difficult to see that it as a material object which can be used to create artifacts. Thus, a novelist has to clean up, scrub, etc. to make his language pure like the sounds that a musician uses for composing music. Here, the writer's task becomes very similar to a sculptor who has to cut, shape and re-shape the material used every day in making thousands of other objects, so that he can create the aesthetic object that he has in mind. The material which the sculptor uses for his statue, be it marble, bronze, wood, would remain whatever they have been in the beginning, and the audience while looking at the statue, will appreciate the craftsmanship, the material beauty, and the concepts which the statue is conveying, so that while looking at the statue, one does not recognize the marble, bronze, etc. but see the statue instead. Likewise, a novelist has to 
"scrub...clean...smooth...polish until we can scarcely recognize the words on the page." In this process the real world associations of the words are not lost, but they are fashioned in the way which fits the author's purpose best.

Gass's common argument in all these metaphors is that treating language as transparent will result in ignoring the physical resources of the novel. But as soon as we realize that language does not merely have a referential role, the way that language is used to create a world of words becomes a major element in writing and reading novels. A step further from foregrounding language, is foregrounding the material body of which written/printed language is a component and though this has been the normal practice for other arts, our habits in reading and the utilitarian aspect of the novel has blinded us toward this. One danger in bringing these physical resources into play is that they might take over the signifying strategies, so a good writer is the one who does not use both the signifying strategies and physical resources "beyond need." 


\section{Case Study 1.1. Willie Masters' Lonesome Wife}

In attempting to describe the book [Willie Masters' Lonesome Wife] it is impossible to invoke the usual categories. It is an assault on our customary orderings. Typographically it is a kind of game. The print varies constantly, lines inflate and grow smaller, tumesce and detumesce; footnotes crowd out the text and corner us; the text divides into two, into three, into more-it cannot be read in a linear way. Clusters of words murmur or shout at us from unexpected places in the margins; asterisks begin to proliferate like some rapidly reproducing organic life until they fall around the pages like stars. There are headlines and placards, even bursts of music. The pages change colors. In every way the texture of the text is constantly transforming itself. (Tanner 183)

Gass's own claim to being a transliterate writer in creating a work which uses "all aspects of print" is Willie Masters' Lonesome Wife in which as Kaufmann demonstrates, he "use[s] the full resources of the physical text from typography to photography" in order to "make the text visible." There are currently four editions of this novel available. The first edition,was first published as a TriQuarterly supplement. The designer of the book, Lawrence Levy says that "Subscribers received the paperback with their subscription, ... we also published an edition of 500 hardcover copies, 50 of which were signed and numbered by William Gass. Knopf published a version subsequently....though not as lavishly (Levy, personal interview). $^{7}$

These editions vary mainly in the colors, paper quality and the front and back cover. The first two editions have been printed by TriQuarterly, the first TQ edition-which is the edition analyzed here-comes in paperback and has a nude photo of a front torso of a woman on the front cover and the buttocks (of presumably the same woman) on the back cover. This image is downsized to a stamp size in the hardcover edition which was again published by TriQuarterly in the same year. This stamp size picture of the nude female torso and buttocks have also been used in the

The full interview is available in the Appendix. 
subsequent Knopf (1971) and Dalkey Archives (1989) editions. The Knopf edition uses fewer colors for the papers, and finally the last publisher published it in a single color. As Levy says:

The book was reprinted by Knopf in 1971 with two different paper stocks, a green-tan for most of the book and a glossy white for the last seven pages (the same as that used in the original TriQuarterly publication). When it was reprinted again in 1989 by the Dalkey Archive, all its pages were the same white paper stock, one less glossy than its original publication's last section. (Levy, personal interview)

As Richard Henry, editor of Willie Masters' Lonesome Wife; a Case Book, an online guide to Willie Masters' Lonesome Wife hosted on Dalkey Archives website, has mentioned, designer of the book, Lawrence Levy, has had no input in the subsequent changes on the front and back cover, the colors and the paper stock. "I know that once the original hardback and paperback were printed, Levy had no input. The subsequent editions, with the move to first a grey heavy stock paper and then to the glossy white throughout were beyond his contributions" (personal correspondence). So as can be seen, the subsequent editions of the novel have been progressively removing one of the distinguishing aspects of the text's metatextuality, and it has been a choice on the part of the publishers rather than the author or the designer.

The reason that this novel has been chosen to be analyzed here is that its physical body displays itself in different ways, the quality of the pages, their colors, and arrangement of words and sentences on them keeps shifting from page to page, its narrative moves back and forth between the pages and its essay-like form makes it more challenging for the reader to follow what is happening, and these features provide a unique reading experience for a reader who understands and appreciates the way this materiality plays a major role in its narrative. On the other hand, the collaborative status of the book, and the other unique characteristics of this book such as colored pages, typography, different reading paths, makes it much more similar to the works of the hypertext fiction which will be discussed in detail in the next chapter. 
This novel also showcases language and a variety of ways in which it is used. There is interior monologue at the blue section, dialogue at the yellow section, apostrophe and warnings in the red section, and a long monologue at the end of the book at white section. Moreover, there are over twenty quotations from other writers has been dispersed all through the novel.

\section{Physical Organization and Design}

The graphic surface of this novel draws attention to itself through a variety of means that will be analyzed here. As discussed earlier, this novel comes in four parts, marked by the four different colored pages (blue, olive, red and white), none of which are paginated. As we will see in the next chapter, sequential pagination is something which is not a usual organizing system in hypertext as well. By not assigning numbers to pages, the book breaks its traditional arranging format and this has provided a challenge from the beginning for those who have written on this novel. Some critics like McCaffery and Caramello have used colors to reference the quotations that they have picked up from the novel and used in their books/essays, others have numbered their own copies by hand and have referred to these-sometimes vague-page numbers. The problem with the latter group is that they have not specifically stated where they have started their numbering, and this confusion lays bare another convention of the print novel which their numbering does not start from the very first page, and if these novels have an introduction (or preface) those might have been numbered in roman numbers. Therefore, for ease of reference, the quotations here will be referred to by the color of the section in which they appear.

\section{Collaborative Status of The Book and the Role of Design}

An important feature which should be discussed here is the collaborative nature of this book. Although collaboration between a writer and a graphic artist is not something new, this one is quite different from the majority of those works because as both Gass and Levy have stated in their interviews, this book required a huge amount of input from the graphic artist. Even though Gass was not quite satisfied with the result, the outstanding design of the book won the "American Institute of Graphic Arts 50 Books of the Year award... which was then the highest American book design 
award" (Levy, personal interview). Here is what Gass has said about this collaboration in 1983 interview by Tom LeClair:

I was trying out some things. Didn't work. Most of them didn't work. I was trying to find a spatial coordinate to go with the music, but my ability to manipulate the spatial and visual side of the medium was so hopelessly amateurish ... and the work also had to go through so many hands, that the visual business was only occasionally successful, and most of that was due to the excellent design work of Larry Levy, not me. (Le Clair and McCaffery 158)

Gass here openly credits Levy for creating the physical body of the book. But how and in what ways has Levy's work changed this novel and what has been Gass's initial conception of this novel. In order to investigate these issues further, the typescripts of the novel have been studied and a separate interview with Levy has been conducted so that their different takes shed more light on this matter.

\section{Typescripts and the Interview with the Book Designer}

Since a great deal of manuscript/typescript changes before it finds its final form in the print body of the text, bringing the materiality of the medium to the foreground requires a close collaboration between the author and designer. This is the main reason that some of the writers in the digital era such as Michael Joyce and Bantock have taken the role of writer-editors and Danielewski moves even one step further and writes his novels in Adobe InDesign software which presents him with a layout which shows how each page will appear in the printed book. As we will see, these inscription technologies become an important part of the creation of the novels and that is why Hayles says that in the third millennium books are "born digital." However, these technological opportunities were not available to Gass in the late 1960s and as we have seen from the previous quotation, his unfamiliarity with the design aspects of the medium was the main reason for his dissatisfaction with the end result. Willie Masters' Lonesome Wife was according to McCaffery "originally a section of a much longer work, never published, on almost every narrative mode ${ }^{8,}$

\footnotetext{
8 "Every narrative mode" which Gass refers to here, becomes more similar to the "multimodality" aspect of the metatextual novels which will be explored later in this thesis as well.
} 
(171) (italics mine). So the closest that one can get in understanding what Gass originally intended the book to be is through studying his typescripts and interviewing the designer of the book, Lawrence Levy.

The typescript of the book which is kept at Washington University in St. Louis is around 459 pages and a study of that revealed that there are at least 11 drafts of varying lengths for the novel. All through the typescripts, there are very few notes about the design issues and most of the changes refer to the corrections and/or adding/removing some words in the text. The earlier versions have much more corrections and there are almost no comments about the graphic surface of the novel in them. The way the text should be arranged on the page becomes more apparent in the final draft and the final typescript has even a few (not all) the coffee-cup stains on it. There are also some general hints about the graphic surface of the novel in those drafts, for instance the words which are printed by a different type font have been underlined. However, there is no specific comment about how this part of the text should appear or what specific font type and size this or that word should appear in. The main implication of studying those typescripts has been what Gass has also talked about in his interview with LeClair that he has had some general ideas about what this or that part of the graphic surface of the novel should be, but most of the decisions related to the graphic surface of the novel (as Levy states in his interview too) has been taken in consultation with Levy, and the book designer here has had a major role in bringing Gass's ideas to life. In Levy's words: “most of the design ideas, including the photography, that are in the present book were developed by me in close consultation with Gass, but were significant departures from the manuscript [sic]" (Levy, personal interview).

In the absence of direction for the graphic surface of the novel, the most outstanding feature is the parts which Gass has eliminated from the later typescripts. Some of these deleted parts include Gass's direct commentary on the language and support the way his ideas have been analyzed here:

Ordinarily language is like the gray inaudible wife who services the great man: an ideal engine, utterly self-effacing, devoted without reminder to its task; but when language is used as an art it is 
no longer used merely to communicate. It demands to be treated as a thing, inert and voiceless. Properties that it possesses accidentally as a sign, it suddenly possesses essentially. (typescript n.pag.)

If you found blank pages in a book you'd bought, you wouldn't feel embarrassed and you might say: hey see what these cheapjakes have done, they have left out the dirties. And you'd want your money back. Painter, too, can leave areas of canvas empty or spread the same sullen color over great swatches of their precious space, daub after daub identical, but do you suppose, having taken a fancy to it, we could run a little bit of catafalque along: catafalque catafalque catafalque catafalque catafalque catafalque catafalque catafalque? Not on your life. Crepuscular has advocates, as does dirigidibility-both nice_-but catafalque is best. (ibid.)

What these quotations and other ones which have been deleted emphasize is the role of language in a work of fiction which is different from the way it is used in everyday context.

On the other hand, the interview with the book designer, the first of its kind, shows a different aspect of this cooperation. As we had seen, despite Gass's dissatisfaction with the design issues he holds Levy responsible for the occasional moments that the visual business - according to him — has been successful. Levy, who had been the art director at TriQuarterly at the time, does not recall any "nutty" idea that Gass might have had for the book, that have had to be abandoned due to technical or financial problems. Nevertheless he is quick to point out the challenges that he has faced while working on this project with Gass: "An additional challenge was trying to understand the ideas originally presented in the Gass ms [sic] and incorporating their intention, if not always their actual look" (Levy, personal interview)

So although Levy does not claim that he has totally incorporated Gass's idea in the design of Willie Masters' Lonesome Wife, and at some point he states that he hopes that the "look of the book was connected to its meaning, which was the point of the design," he is the one who has taken Gass's ideas for the design and created the book in the form in which it exists today. Since Levy has been approaching the book 
as a material object — which its design is directly connected to its meaning - he is quite aware of the purpose of this book to foreground its materiality, he believes that the "use of distorted typography, a variety of typefaces, nude photographs, different color paper stock... which calls attention to the book as object," has been the main idea of the book and that is what distinguishes this book from the rest. However, this collaboration at its best seems to be have been a compromise between a writer who is well aware of the role of physical resources in his book but his ability to manipulate them has been "hopelessly amateurish," and an art director/designer who is aware of the relationship between the physical resources and signifying strategies in the objects that he is designing and is "experienced in the mechanics of typography and printing and binding." Moreover, Levy has worked closely with authors on several essentially innovative typographic books which had unique design problems" (personal interview) and as is expected, his approach to the design and what ideas can be used for it has been more practical. Though Gass in the end credits Levy's efforts for the success of some parts of the novel, he goes on to openly admit that the whole book has failed. Despite Gass's admission, the book won several awards, went on to be published by two other publishers and is still available for purchase, became popular with the critics and the number of essays published and referred to this novel-Google Scholar finds over 16,000 results for it—shows that through Levy's design, this book has survived the test of time and as Levy puts it, its design seems innovative even for the early 2010s: "for the most part, books still don't do much of what Gass was trying to do, and still look pretty straight forward" (Levy, personal interview).

\section{Covers}

The nude torso and buttocks of the woman on the front and back cover of the book make the claim that the body of the book is like a body of a woman which needs to be caressed and its beauty appreciated, rather than being taken for granted and merely used for sexual pleasure. In the same fashion, our insensitivity to the sound, shape and form of the language makes us similar to people who instead of "caressing" the words and appreciating the beauty of language in a work of fiction, focus on their utilitarian aspect. In this way, our relationship to the language becomes more similar to relationship of a man to a prostitute. Such an approach leads to the 
language-as-a-whore metaphor which has been commented on by Gass. Being asked about Babs, the narrator of Willie Master's Lonesome Wife, in one of his interviews, Gass replies:

She is language in a way. ..... In other words, she comes out of a metaphor. If language is the medium, then I can set up the standard relation of the author to the medium as to a lover or a prostitute or whatever. And you really have to make her a kind of prostitute because language is available to anybody. (Gass, interview by Castro 33)

The relationship that Gass talks about would be highly dependent on the way the authors (and readers) treat language and in this book and how they expect their readers to react to the highly visual aspect of the book.

\section{Colors}

Colors are one of the most distinctive features of this novel and make this novel appear unique even in the company of other experimental novels. These colors and their sequence can stand for various things in the novel. Some critics believe that they are the stages of intercourse, stages of body, etc. However, all of these interpretations take one important aspect of this novel totally for granted: emphasizing the materiality of the book through colors. As Gass observes in On Being Blue (1976) color has had the same fate as the language; colors have always been meant to generate a constant set of values rather than being seen for what they are. According to Gass “seldom was blue for blue's sake present till Pollock hurled pigment at his canvas like pies" and "a color's unity is inherent ... every color is a completed presence in the world, a recognizable being apart from any object" (On Being Blue 72-74).

Color for Gass is a "recognizable being" an existence that is easily identifiable wherever it appears and does not have a pre-determined form. This formless existence of color makes it take the shape of the physical form in which it appears and creates a new object through its appearance in that form. However, before Jackson Pollock uses color in non-representative way in his paintings and by doing this frees the color "from objective context ... [so that it] becomes the subject in itself," there was a 
different approach to color. It did not have a separate entity away from the object in which it appeared which stood in sharp contrast to the way Pollock and many other painters after him used it. As Gass quotes an art historian, color has been used to "serve rapid recognition and identification, facilitate the interpretation of shapes and the articulation of masses and accelerate the perception of form, or tactile values, or movement" (75). ${ }^{9}$ This resonates with the way that language had been used in works of novelists whom Gass has called "preliterate." So in the same way that Gass believes colors are "completed presences" in this world, and for him color is not an aspect of a real object but must be appreciated for what it is, not what it represents, language must be appreciated for what it is in a work of fiction not what it represents. This claim is supported by the way the narrator in Willie Masters' Lonesome Wife uses words like catafalque, not for what they refer to, but for the form and the sound that they have and he makes a couplet out of it.

\section{Photos}

Next to colors, another element in the novel which brings its visual mode to the foreground is the use of photographs in the novel. What was said about the colors in the previous section can be said about the nude photographs of the model, which have been inserted in various parts of the novel. These photos could not possibly belong to the narrator of the story, because as Gass mentions in his letter to Wolfshohl, the model is "far too young" for the way he had imagined her and also there are textual references in the text which justify Gass' dissatisfaction with the model. In the blue section, Babs says: "Until my flesh began to lose its grip, I danced in the blue light with the best, and then I married Willie" (blue). However, these photos show a young woman who is slim and there is no sign of her flesh beginning to "lose its grip." None of the photos are in a vividly inviting or erotic pose, and what these photos aim to do in this book is again to foreground the materiality of the book and their own status as objects of beauty which should be seen and appreciated rather

\footnotetext{
${ }^{9}$ Gass stands in sharp opposition to all these functions, as can be seen by his comment on this quotation of Berenson: "Again and again we strike the same bigotry about the blue, the same confusion of categories, the same errors of mind... and the same disastrous lapses of taste" (75).
} 
just a means to eroticism. ${ }^{10}$ In other words, the photos and their placement in various parts of the novel do not materialize the image of Babs in the novel. Therefore, they are not the visual equivalent of what the words are describing. Instead they show a clear resistance toward such a reading. That is why the only captioned photo in the book (at the last page of the blue section) asks "my Mister Handsome how could you?" This rest of this question could be "how could you only see me as an object of pleasure, and not see the beauty of my own body?"

\section{Typography}

Experimental typography has been a major tool for the writers who want to stress the materiality of their work from the early decades of the twentieth century. According to Johanna Drucker, Mallarme was the first poet to use experimental typography in his work A Throw of the Dice (1914). As she shows in her book The Visible Word (1994), many poets in the twentieth century have used experimental typography to highlight the materiality of their work and the material form, from which it is made. For these poets there has been a direct relation between transparency (of the language) and the immateriality (of the work of art):

The notion of linguistic transparency implies immateriality, that which is insignificant in its materiality, to which nothing of linguistic value is contributed by the form of the written inscription which serves merely to offer up the "words" in as pure and unmediated a form as possible. The act of repression on which this notion depends is monumental, really, since it requires continual negation of the very evident fact of the existence of what is immediately before the eyes in the name of its signified value. (14) (italics in the original)

Twenty different typefaces used in Willie Masters' Lonesome Wife can be said to have the same role in foregrounding the materiality of the book too. Although different typefaces have been used in different eras of print fiction, they have been meant to be even more invisible than the words. As the type designer Tobias Frere-Jones says:

\footnotetext{
${ }^{10}$ To read more on the anti-pornographic stance of Willie Masters' Lonesome Wife see 'Willie Masters' Lonesome Wife as Pornographic Critique' by Rolf Samuels on the Dalkey Archives Webpage.
} 
The sort of classical modernist line on how aware a reader should be of a typeface is that they shouldn't be aware of it at all. It should be this crystal goblet there to just hold and display and organize the information. But I don't think it's really quite as simple as that. I think even if they're not consciously aware of the typeface they're reading, they'll certainly be affected by it, the same way that an actor that's miscast in a role will affect someone's experience of a movie or play that they're watching. They'll still follow the plot, but, you know, be convinced or affected. I think typography is similar to that, where a designer choosing typefaces is essentially a casting director. (Helvetica)

There could have been even more fonts in Willie Masters' Lonesome Wife if the costs and the technology of the time had allowed. Gass in his letter to Wolfshohl has mentioned that "I indicated in the margin of the ms [sic] that I would clearly love to have each quote set in the type of the first ed. There would be a kind of little history of typography in the book."

This "typographical play" of the book makes the materiality of the book more visible by resisting the "reading convention to see through print to what it references." According to Gass, this typographical play "tends to make the sign increasingly concrete, particular, and present, which runs counter to the nature of a sign. Signs are supposed to call attention to their referents and their meanings and run away" (letter to Wolfshohl).

\section{Footnotes and Asterisks}

Footnotes are not very common in works of fiction, but they can be seen marching from page to page in the olive section of the book. Apart from their relatively huge number (twenty five footnotes in twenty four pages), these footnotes are not designated by numbers, as is the normal practice in the other books, but they are shown by asterisks, which make it more and more difficult to follow them while they form longer lines with more asterisks. Also these footnotes which are always meant to be in the bottom margin of the book, gain more attention as they move further and further up the page, to the extent of pushing the main part of the text (the 
dialog between Ivan and Olga) to the top of the page and finally moving it to the margin of the book, as they become larger and larger, finally the next page is totally invaded by asterisks, according to Karen L. Schiff:

Gass transforms asterisks from textual punctuations into visual images, and thereby asks readers to redefine their perceptual expectations of these marks...In contrast to the line of twenty-five typographical asterisks that lead into a footnote at the bottom of this same page, these asterisks no longer function as punctuation: they create a field of visual impact. The reader's attention is drawn to their aesthetic properties instead of their signifying power. As Gass writes five pages earlier, "Anyway, these asterisks are the prettiest things in print"....they bring the reader out of a narrative reverie and into the present moment's experience of looking at the page as a visual object. (digital case book)

Therefore, by having "visual" aspects, these asterisks become devoid of the referential role to which they have been usually assigned, and in this way as we will see in the mirror section, refer to nothing else except themselves. This point is even further emphasized by the whole page of big asterisks which appears in the olive section.

\section{The Mirror Page}

One of the textual features of this book which is located at the beginning of the novel is the mirror page. The verso page reflects the recto page in a mirror style without adding or reducing anything. This mirror page is an early reminder that this novel is like a mirror not a window, therefore it would be impossible to go beyond this mirror and only reflects back its own materiality. Other critics, such as Kaufmann have discussed this mirror page in relation to the way Stendhal had described novels, which is "a mirror carried along a high road." Stendhal's context for that metaphor is defending the novel against those criticized the novelist for writing about the hideousness of the society and tries to rebuff this attack, but in doing that it aligns itself with the same tradition that does not see the physical body of the print novel and only assumes a referential role for it. In this sense Stendhal's 
mirror is interchangeable with something like a video camera recorder, but Gass's mirror page is unique because it rejects those ideas and depicts the physical body of the novel for what it actually: ink and paper.

\section{Coffee-Cup Rings}

The coffee-cup rings according to Kaufmann are "the most blatantly metatextual aspect of the book." There are altogether eight coffee-cup rings in this book, with the first one being at the bottom of the first page of the red section, which starts like this: "The muddy circle you see just before you and below you represents the ring left on a leaf of the manuscript by my coffee cup. Represents, I say, because, as you must surely realize, this book is many removes from anything I've set pen, hand, or cup to" (red).

As can be seen in this quote, by placing these coffee rings on various parts of the page, the writer aims to emphasize the non-representational role of his novel and how real-world objects become something totally different when they are mediated in his work. In this sense, the coffee-cup rings, as Kaufman also observes, become similar to the photographs in this book. One of the coffee-cup rings at the top of the last page of the red section emphasizes this aspect quite openly. Within this coffee-cup ring, there is a sentence "this is the moon of the daylight." Since this novel abandons the referential role and instead underlines its materiality, the traces or the objects of the outside world which are used in its construction can have totally different roles. Gass discusses this transformation in one of his essays; "Carrots, Noses, Snow, Rose, Roses." In that essay he discusses that when a carrot is placed on the top of a snowball "does not simply stand for or resemble a nose, it literally is a nose now-the nose of a specific snowman" (Gass, The World Within the World 288). In the same way, the elements such as photographs or coffee-cup rings which come from the outside world when placed in a metatextual novel in addition to standing for what otherwise is absent, can be used as a component of the novels and find any meaning that the novel assigns to them.

"The aim of the artist ought to be to bring into the world, objects which do not already exist there and objects which are especially worthy of love" (Gass, Fiction and Figures 284). 
"For example, suppose there were imprinted here, as in letters of love, a pair of lips; could you, by kissing them, let the paper pander between us?.... All contact — merest contact — any contact — is impossible" (Gass, Willie Masters' red).

The quotation from the red section of the novel brings an important issue to the foreground, that a novel as a work of art which is an object created by the author, is all that there is between the reader and the writer. The writer cannot reach his/her audience through it because any contact is impossible, so in the absence of the writer, a novel should be autonomous in the sense that what it does or what it says should not be dependent on its author. Therefore, all these features that have been discussed above, should not be examined by their presence outside of the novel but by the way they have found new meanings in the print novel as an independent object in the world.

\section{Narrative Strategy}

It follows that narrative can play a variety of games with its supporting medium: it can go with the medium and fully exploit its properties; it can ignore the idiosyncrasies of the medium and use it purely as a transmission channel; or it can actively fight some of the properties of the medium for expressive purposes. (Herman, Jahn, and Ryan, 290)

When it comes to narrative, this novel lingers between several layers of possible narrative and essay. Towards the beginning, there are several narratives which may or may not be related to each other, but in some other parts, especially toward the end of the novel, the essay aspect takes over and the books becomes an essay on language. Because of this feature of the novel it would be hard to construct a single narrative for the whole book, which expands all over the book. However, another approach which is more rewarding is identifying the narrator at each section and exploring her relationship with the metatextual body of the book in which it appears.

\section{The Narrator}

Who is the narrator and how does her identity affects the narrative that this work tries to construct? This question plays an important role in the way narrative is 
being constructed bit by bit by the text, because almost all through the novel she addresses the reader and promises to be "passionate and beautiful" if she is treated with warmth and attention. In an interview Gass had described her as a "woman of easy virtue" (typescript) which is the same as language which he identifies her with in a later interview, and this is the lead that several critics have followed. McCaffery calls her "lady language herself," Charles Caramello (1983) moves one step closer by saying that the narrator "appears to be imagination performing itself in a book." Similar to Caramello, Arthur Saltzman also brings the materiality of the medium into the mix, according to him the narrator is "an amalgamation of narrator, story, and the physical book itself" (qtd. in Kaufmann, 128). Kaufmann himself provides a useful analysis: "She might more properly be thought of as Fiction or the Novel, rather than as 'language' or 'imagination.' Language is her body, her embodiment, while imagination brings her forth." (128). All of these interpretations, except Saltzman's, treat the narrator as a fixed identity through the novel. But similar to the skits that she writes and acts in, she changes in different parts of the novel and takes the form which the critics above have described. However the most prominent identities which she finds are the language and the physical book. It is essential to note that these two identities both overlap in various parts, and since language is instantiated in the print medium, it is quite easy to associate one with the other, but the differentiation between these two becomes important when other media such as digital media provide material support for language.

\section{Mini-narratives}

The mini-narratives of Willie Masters' Lonesome Wife support the issues raised by the textual structure of the book. Despite its small size there are several narratives moving alongside each other in different parts of the novel. There is no overarching narrative over the four colored sections, but each one contains mini-narratives in different forms which will be studied in detail in the rest of this part. Since Gass is denying a representational role for the novel that he has created, the story of the novel becomes the story of its own materiality which in other words, is the story of the materials used to create this novel: language and the print medium in which it is instantiated. Reading narratives from this perspective makes it easier to understand why there are several mini-narratives and applications of language, for 
instance quotations, within the novel. Similarly the narrator's obsession with touch and physical contact and long soliloquies become more understandable, once one realizes how it keeps switching between the language and the physical book. In what follows a few of these mini-narratives from different color sections are analyzed to shed further light on how the narrative of the book supports and enhances its physical organization.

\section{Blue Section}

The blue section starts with the image of a woman about to swallow the letter $\mathrm{S}$, this section contains three narratives which are differentiated by roman, italic, and bold type face. The section in italics as Holloway has observed is a "third person account of the narrator's youthful sexual activity." The second narrative in roman letter is where the narrator talks about present and the naming of the parts of the body. Finally the last narrative in bold type is where the narrator talks at length about spit, and kiss. It is not hard to see the way the first and second narrative here can be connected to the issues discussed above (touch and language respectively), but the third one which will be analyzed here might not see directly connected to either of these. The narrative in this section starts with: "Suppose, for instance, a stranger were to - oh, say, you're laughing uproariously, and that's the occasion for it - spit in your mouth, god forbid. Still they do worse" (blue). The unpleasant predicament that the narrator wants us to suppose, does not seem to be directly related to language, but spit can be the closest metaphor for language, because both of them come out of our mouths and we when we reiterate what somebody else has said, we are actually spitting out the words that $\mathrm{s} / \mathrm{he}$ has put in our mouths. As we have also seen from one of the quotations from Gass, he has the same approach to language: "He [a writer] may pretend that every syllable that he speaks hasn't been spit, sometime, in someone else's mouth." Thus, here the narrative is the narrative of the language, but it does not take long before it becomes the narrative of print book. Therefore when the narrator is commenting on the way she cannot kiss the reader, she is referring to the materiality of the book:

When I should touch them [rubbered tips], he should feel the rush of my caress across the whole length of his cripple's sticks as though they 
were his only avenues and scopes of feeling....so when I should touch them, give my kiss, it would be as if I'd made him see my love entire in a vision. (blue)

This theme of kiss and ultimately the impossibility of contact between reader and writer returns again in the red section as we will see later, when the narrator again takes the mantle of the physical book.

\section{Olive Section}

The olive section which is the longest section of the book is in the form of a skit with several footnotes. It is possible to divide this part into four parts. The first one is stage directions that prelude the play, this part is marked by several footnotes, and narrates the background story for the two characters who are on the stage. The representational aspect of this section is broken when in one of the sentences where two options are provided for blank space in there. In fact since the writer has not chosen any of them and places both words above and below the blank there are two options for reading this sentence. Here is what it looks like

\section{what it is. The audience, of course, is having fits. darkest steepest Slowly, ever so slowly, slowly in the way light rises a \\ erases the \\ swallows the}

By leaving these two option open, the text shows that the process of creating a narrative through arranging words after each other, is a process of selection and omission, and every choice requires abandoning the other choices that could have been equally fitting in this context. So here the text does not prioritize one over the other and allows both of them to exist literally parallel to each other.

After this part comes the dialogue section which is quite short and is interrupted by the asterisks and footnotes time and again. However, interestingly enough this short dialogue focuses on something which is not expected for a light-hearted skit which this part tries to depict; naming the objects and people which creates linguistic identity for them. The male character, Ivan, cannot remember the 
name of his "missus" and calls her by four different names before finally remembering her name. Right after that they get into a discussion in which its most distinctive part is the wordplay they have:

Ivan. ${ }^{11}$ You appear to have baked somebody's, eh ... thing-gummy, dear, in your, ah ...bread.

Olga. Buns!

Ivan. Buns

Olga. There's only bread in my buns.

Ivan. My bun's not entirely bread.

Olga. Bun!

Ivan. Bun.

Olga. Your bread's a bun.

Ivan. Well, ah, it's not wholly, that is to say, not altogether, straight through, bun

Olga. Not a whole bun?

Ivan. No mum.

Olga. Better be one.

Ivan. Yes hon.

Olga. All bun.

Ivan. Then there's another bun in my bun.

Olga. Another bun?

Ivan. Yes hon.

Olga. Bun in your bun?

\footnotetext{
${ }^{11}$ I have followed Gass's punctuation in this section, he uses a single dot, instead of the conventional colon.
} 
Ivan. Yes hon-but not a bread bun.

Olga. The bun in your bun's not a bread bun?

Ivan. No mum.

Olga. A bun's a bun, it must be one.

As can be seen the whole point of Ivan's complaint is almost lost in the verbal exchange between the two which is full of linguistic misunderstandings. What this absurd dialogue highlights is the inner capability of language and how instead of being an empty vessel it can complicate the act of communication through its nuances.

There are a variety of quotations from other writers within this section and the red section. The narrator also comments on this multitude of quotes by saying that "the whole of literature lies before us" (olive section). Some of whom like Tolstoy and Shakespeare are more well-known, but some of them like Sheikh Nefwazi are not as famous as others. As Gass has stated in one of his interviews he intended to bring in every quotation with the original font type in which it has been first published. Had he done this, Willie Masters' Lonesome Wife would have been a history of print with samples from almost every era. These quotations contribute to the foregrounding of narrative as a linguistic construct and how the linguistic claims to factual side of the world are in fact fictional. For instance the quotation from Nefwazi- "Do not drink rain-water directly after copulation, because this beverage weakens the kidneys,"(qtd. in Willie Masters') - is from Perfumed Garden a fifteenth century Arabic sex manual. The instructions in this book, such as the above sentence, have been treated as facts for some people, but for a modern day reader who does not drink rain water, this sentence does not hold any factual weight and is impossible referentially but quite possible to write it in a work of fiction, as the quotation by Sterne "a cow broke in tomorrow morning to my uncle Toby's fortifications" also shows. So by losing its referential value, it becomes merely a linguistic construct that finds meaning in the wider context of language, because as Kaufmann has observed: "in fiction, the reader is warned, anything can happen, even things that have not happened yet in the past" (96). 


\section{Red Section}

The red section keeps its graphic surface in the foreground by introducing the several layers of narrative on most of the pages in this section. It starts with one narrative, but then the narratives on each page gradually increase and some of the pages in this section have up to four narratives. The four mini-narratives and one play here continue the narrator's focus on the language and the materiality of the print book. In the first mini-narrative here, the narrator openly comments on the coffee-cup rings which appear here and in the white section: "The muddy circle you see just before you and below you represent the ring left on a leaf of the manuscript by my coffee cup." This opening statement to this section becomes a springboard for a long piece of essay-like narrative about the impossibility of the literal and referential aspect of the novel. The implications of this non-referential role is expressed in other narratives in this section; when the novel ceases to be a window, it becomes a wall which divides the narrator from the readers, "a wall divides us... all contact-merest contact - any contact - is impossible, logically impossible." Also in the same section the word "catafalque" which had been repeated several times in the blue section again appears and its presence is another reminder that the words along with the colors and other textual features do not need to have a referential status to appear in a work of fiction. It is their own sound or appearance that would justify their presence there. The narrator believes that it is now high time that the writers stop worrying about the meaning of the words that they are using, as the narrator says: "the writers lack the luxury of painters who use their medium without worrying about its meaning" (blue section).

\section{The White Section}

The white section is the smallest part of the book and has only eight pages. This part which is completely in white, maintains its difference from the pages of the conventional fiction by being printed on "thick, high-gloss white paper like that of expensive magazines" (McHale 173). In this section the narrator becomes language and and highlights her own role in fiction further. "When a letter comes, if you will follow me, there is no author fastened to it like the stamp; the words which speak, they are the body of the speaker." Therefore the text finds an identity separate 
from its creator and in this new identity the figurative way that the language is used-as opposed to the referential way in which it is used in non-literary context-becomes the most important feature of the work: "Then let us have a language worthy of our world ... metaphor must be its god, now gods are metaphors." Finally the naked image at the end of this section, which is at the end of the book too, brings back the image on the cover of the book and by completing the cycle, shows that no reading is complete.

\section{Reading Strategy}

"The purpose of a literary work is the capture of consciousness and the consequent creation, in you, of an imagined sensibility, so that while you read you are that patient pool or cataract of concepts which the author has constructed" (Gass, Fiction and Figures 33).

The reading strategy becomes a very challenging aspect of this book, because the lack of overall plot and the writer's emphasis in attending to the material qualities of the book, in addition to the way the narrator stresses this on almost every page of the novel, make it harder to read for a reader who is accustomed to the narrative in the conventional novels. There are several points in which the narrator pokes fun at such a reader and finally goes on to address the reader by saying that "you have been had, from start to finish" (red). But for a reader who is responsive to the characteristics of the print medium which are highlighted here, the book as the narrator says in the white section becomes something else:

I am that lady language chose to make her playhouse of ... though you'll not be back, your brother will. Tell him he is responsible for me, and that I give as good as I receive. If he will be attentive, thoughtful, warm and kind, I shall be passionate and beautiful. (white)

As we have already seen in the comment that Schiff had made about the asterisks, those marks "bring the reader out of a narrative reverie and into the present moment's experience of looking at the page as a visual object." Schiff's comment can be used for the whole reading strategy for this novel, because this is exactly the demand that the book is making from the reader. The reader, on the other hand, can 
react to this feature of the book in a variety of ways. An experienced reader of conventional novels whose experience has taught her to ignore the physical resources of the book completely, could find this book frustrating because it is encouraging her to do what conventional print novels have been asking her not to: seeing the material body of the print novel and drawing her attention to the visual aspects of the book itself.

This heavy demand that this text makes on the reader, has been one of the reasons that apart from Kaufmann — and at some points Caramello —all the other critics who have written on this novel, have failed to take the materiality of its medium into account and have resorted to conventional methods of literary criticism. The strong defiance toward a conventional reading becomes quite vivid when the narrator directly addresses the reader who has failed to read it in the way that it asks to be read

You've been had, haven't you, jocko? you sad sour stew-faced sonofabitch. Really, did you read this far? puzzle your head? turn the pages this and that, around about? Was it racy enough to suit? There wasn't too much plot?... Is that any way to make love to a lady, a lonely one at that. (red)

From the beginning of the novel the reader has to make a choice about the colors and how they affect her reading, the nude photos and the narrative of the first kiss in the italic section, in addition to the erotic connotations of the blue, suggest that it could be the early signs of an erotic story to come, it does not take long before these expectations are overturned by the narrative. These elements within the narrative according to Brian McHale:

... often appeal to their readers' "lowest" instincts... The aim of such sensationalism is to lure the reader into making an emotional investment in the sequence under erasure, typically by arousing his or her anxieties, fascination with the taboo or prurient interests. Having become "involved" in the representation, the reader thus resents it when the representation is de-represented, erased. The reader's impulse to cling to the erased sequence heightens the tension between (desired) 
presence and (resented) absence ... the use of pornographic or quasi-pornographic material for this purpose is the clearest example. $(102-3)$

Therefore once again, what seems to be an erotic book based on its front and back cover and its title, proves to be quite contrary to what it pretends to be. Moreover, in order to emphasize this difference, one page of a well-written erotic story with elaborate metaphors appears out of nowhere in the olive section and is the only page in the novella which follows the conventional format of pagination, has the same font size all through the page and all the other criteria for a conventional novel except the color and quality of the page. This playful attitude of the novel makes the reader either dig deeper to find some proof for her initial interpretation, as McCaffery and Caramello have done, or try her own reading according the expectations that the book makes upon the reader. The latter would find that the experience of reading this novel requires paying particular attention to the body of the text that she is reading and this novel expands the expectations of the reader about how novel can be if it is emancipated from its conventional arrangement.

The un-numbered pages of the novel and the arrangements of the chunks of narrative on the pages, especially in the red and blue section, anticipate what later becomes known as multi-path narrative. In order to follow these mini-narratives, the reader has to go back and forth to several parts of each colored section. Although it is possible to read the whole novel in a linear fashion, but such a reading would not be as meaningful as the reading which moves back and forth to follow one specific strand of narrative.

Finally if a human body is made of organs and other functional parts like breasts and arms, a textual body is also made of functional parts, like words, quotations, images, colors, fonts etc. Therefore this is the reason that Gass tries to include all forms of writing in his novel. 


\section{Conclusion}

Thus it can be concluded that this specific work of William Gass gets more similar to the works of the first generation of hypertext fiction which will be studied in the next chapter (Michael Joyce quotes a sentence from Gass' On Being Blue for his hypertext novel, Twelve Blue). However despite all those similarities Gass denounces electronic textuality in his article "A Defense of the Book" on his now-familiar line of reasoning that the "they have no materiality, they are only shadows and when the light shift, they'll be gone. Off the screen, they do not exist as words" (A Temple of Texts 165). Looking at it from Gass's perspective, it would be impossible to see the materiality of the electronic textuality. Gass's view exemplifies something which is referred to by Johanna Drucker in her essay "Intimations of Immateriality" as "a fixture within the popular imagination" (152). Drucker does not specifically refer to Gass, and explores the broader context in which "immateriality" has been accepted by both literary scholars and writers as one of the aspects of electronic texts. In that essay, she states that "immaterial' text of electronic environment has become the fixture within the popular imagination" (ibid). What Drucker has observed is quite important because it highlights a deep-rooted perception of the transparency of the medium in print fiction, which was discussed above, has continued in a way into the whole realm of "electronic environment." However, despite Gass's discussion, the texts in digital media do have material body. This issue will be explored in detail in the next chapter. 


\section{Chapter Two: Hypertext Fiction, and the Remediation of Experimental Print Fiction}

As more people buy and do not read more books than have ever been published before, the book is merely a fleeting, momentarily marketable, physical instantiation of the network. Readers face the task of re-embodying reading as movement, as an action rather than a thing, network out of book (Joyce, Of Two Minds 11).

There is no Platonic reality of texts. There are only physical objects such as books and computers, foci of attention, and codes that entertain attention and organize material operations (N. Katherine Hayles, $M y$ Mother Was a Computer 97). 


\section{Introduction}

In this chapter, the early development of hypertext fiction will be approached from the perspective of the materiality of the digital text, with the purpose of demonstrating the extent to which the aims of the experimental novel in print form affected the initial conception of hypertext fiction. However, instead of following up the convergence discussion and how and in what ways it was critiqued, this chapter follows an alternative genealogy which considers hypertext literature as the continuation of experimental print literature. Although such a claim was made in 1987 by Bolter and Joyce, it had not been widely discussed in academic and scholarly works on hypertext. The notable exception is Loss Pequeno Glazier who in his discussion of e-poetry builds his main argument on this premise. Nevertheless, Galzier's discussion diverges from this research in crucial ways which will be discussed later.

As can be seen from the quotation by Hayles at the beginning of this chapter, the conception of the invisibility of the medium and its neutral role in transferring the message from the consciousness of the writer to the reader(s) perpetuates the idea of "platonic reality of texts" which is rejected by both Gass and Hayles. "Platonic reality of texts" assumes the pre-existence of texts independently of the medium in which they are instantiated, whereas both Hayles and Gass emphasize the existence of texts as material objects created from combinations of media and signs. This is one of the reasons that for both Gass and Hayles, the materiality of the book as a physical object matters. For both of these critics, the meaning of a (fictional) text emerges through reader's engagement with its materiality which in itself is the interplay between its signifying strategies and physical resources. These two key terms though do not ever simply sit as fixed separate categories. A resource for a text is a mode, like the genre of the novel or the alphabetic organization of a dictionary, providing a framework of organizing or structuring principles/conventions which are not either provided by the signs as such, or by the medium as such. This is where mode and strategy are connected, both being organizational in their functions. The physical resources and signs are distinct but it is the way that they combine that generates a text and one should always have in mind the different things which they do in different contexts. It is the instantiation at each given moment or, as we will see in more details later, "the 
moment-by-moment experience of reading a text and our perception of the organizing and controlling structures of the text" (Bolter and Joyce, 45) that create a text, because all of these elements are working in combination with each other and offering an account of that at any given moment in the text is a sign of the complexity of this relationship.

This is important because it brings out the dimension of time in which a text unfolds and the reader's experience of it. In the case of fiction, this is both represented and signified by the narrative. Moreover, the physical resources and signifying strategies are always a blend of these two, it is not just that the physical resources have to do with the graphic surface and signifying strategies just refer to signs, because signs in themselves are a physical resource. Since language is a physical source, the graphic surface can work as part of the signifying strategy. The page surface can interfere with the reader's expectation of how writing appears on the page, and therefore it can highlight some sign values attached to it, and in this way defy the perception that it is a neutral entity for the whole text.

This is also the reason that as soon as a new medium with a different set of physical resources joins the competition, the traditions of print which have been formed over the years in order to "arrange words on a page and pages in a book, in the usual neutral way" (White, 1) become more visible and thus can be challenged or questioned. The new medium, as we will see, seeks to remediate the older medium, but as soon as those traditions appear in a new medium and show that they are not intrinsic to the older medium, their arbitrariness is highlighted and they make us realize that it is necessary to see them as traditions established and embedded over time rather than the fixed affordances of the older medium.

As soon as we adopt the position that "there is no platonic reality of texts," and a text as a physical object is created through its medium, then it follows that the medium plays an active role in forming and shaping a text's meaning. Similar to Gass, Hayles opposes the idea of the transparency of the medium and demonstrates how the physical resources of a medium contribute to creating the meaning of a text through their interplay with the signifying strategies which a reader has acquired and learned to use during his previous engagements with specific kinds of texts. However, Hayles 
takes Gass's discussion about materiality of the book one step further and expands it to the whole realm of inscription technology, regardless of whether the graphic surface is provided by a book, stone, or a computer.

In order to demonstrate how the works of hypertext fiction expose their materiality in presenting their narratives, we need to undertake a comprehensive analysis of the materiality of the digital medium and how it is to be distinguished from print. When the digital materiality is further explained, it will be possible to divide the expansive field of hypertext fiction according to the developments in the materiality of the works and how each generation of these works takes advantage of the range of possibilities that the material medium in which the works are instantiated provides for them. Thus, this section will be immediately followed by a brief discussion about the generations of hypertext fiction.

Writing a novel in the new medium and presenting it as a work of fiction surely must have required a significant amount of (pre)meditation about narrative, new medium and the way its materiality should or should not play a significant part in the narrative, and this is what Joyce confesses to have done during the preceding years of writing the very first work of hypertext fiction; afternoon (Joyce 31-36). Thus, it will be informative to read what Joyce "really wanted to do" and what has been the conclusion of all that reflection on hypertext fiction and afternoon.

Joyce's first work of hypertext fiction is arguably the most discussed work of early 1990s and the main reason which it has been chosen for this research is that this novel in various ways marks the transition of the novel from print to digital media. Furthermore exploring afternoon reveals how this novel changes when its material support as well as its reading and writing software, Storyspace, go through various updates. Those updates practically make a floppy diskette designed to be read through Storyspace 1.0 on a Mac LC, unreadable on an iMac with Storyspace 1.3.0. afternoon also helped to create and establish the characteristics which came to be known as the characteristics of hypertext fiction during those years and in this respect it is a formative text for a study of the materiality of the hypertext fiction. 
This chapter will then move on to a media-specific analysis of afternoon considering how afternoon takes advantage of its new-media capabilities and how and in what ways are its physical organization and design, narrative strategy, and reading process similar to or different from metatextual print novels. The same features will be observed in Fax Messages from a Near Future, a print work published in the early 1990s to demonstrate how print fiction has reacted to the arrival of the new media. Since this novel makes direct use of new communications technologies in print, it can be regarded as an example of the ability of print medium to represent media other than itself.

\section{Hypertext as Continuation of Metatextual Print Fiction}

It was usual in the first phase of hypertext fiction development for critics to claim that it represented a complete break with print fiction and exemplified the new critical and philosophical positions which became distinctive of postmodern conceptions of textuality. But there were writers who took a different view-which it is my purpose to explore more fully - that the hypertext novel is a continuation of the experimental print novel into the digital medium. These writers do not specifically use Kaufmann's term, metatexuality, to describe the link between experimental novels and the hypertext fiction, but a closer analysis of the way they explain this continuation shows that this is the direction that they are moving toward. For example, writing in 1987-the same year in which Michael Joyce finished the first work of hypertext fiction-Bolter and Joyce observe:

by disrupting the stability of the text, interactive fiction belongs in the tradition of experimental literature that has marked the twentieth century....All of these writers [Woolf, J Joyce, etc.] were trying to set up new relationships between the moment-by-moment experience of reading a text and our perception of the organizing and controlling structures of the text. In this sense, hypertextual fiction is a natural extension of their work, redefining the tradition of modernism for a new medium ("Hypertext and Creative Writing" 44-45).

Joyce and Bolter state the case here for modernist experimental print fiction, yet as this thesis will argue, hypertext fiction is a more logical continuation for 
metatextual print fiction. There are two important points in this quotation: the first as mentioned above, locates fiction as a continuation of experimental print fiction; the second which follows from this, narrows this genealogy to modernist fiction. The "tradition of modernism" with its specific attention to materiality of the medium in which it is presented is the same metatextual trend that Kaufmann had identified in the works of high-modernists and in the afterword to his book identifies in the works of early experimental postmodernists:

Gass's self-professed, high-modernist attitudes do not coincide with that of most other postmodernist writers but the metatextuality of their work reveals their kinship. Barth, Gass, Federman, and the like all belong to what could be called early postmodernism. Early postmodernism grows out of high modernism - as its tendency toward metatextuality shows. Early postmodernists view and even assume, at times, the attitudes and gestures of early and high modernism ironically. The contradiction between the formalist and modernist ideals of the book and postformalist and poststructuralist ideals of the "text" that Charles Caramello finds in postmodernism, then, applies not to postmodernism as a whole but to these writers in particular. Metatextuality reveals a division between writers who focus on aesthetic and those who focus on the subject matter of their works. The division corresponds to Charles Russell's notion of two sorts of postmodernists: those who focus on technique or "hermeneutic modernists" (early postmodernists as I refer to them) and those like Marquez, DeLillo, Kundera, and Coover who examine the "severely problematic interaction of personal and collective reality" or "avant-garde" postmodernists (or late postmodernists).

Early postmodernism includes writers such as Vladimir Nabokov, Walter Abish, William Gaddis, B.S. Johnson, and Julio Cortazar - all of whom experiment to one degree or another with the physical composition of their works. These writers, and those mentioned as early postmodernists above, reshape binding, chapter, and page, sometimes abusing or overusing conventional arrangements 
$\ldots$, or at other times overthrowing convention completely and constructing a book that "like a cloud ... changes as it goes"... Each book becomes a performance in Gass's sense. (106-107)

It goes without saying that there are no clear-cut boundaries and some works are better positioned at the borders of modernism and postmodernism, but, as Kaufmann in the above quotation explains, the "kinship" between a writer who occupies a transitional status between modernism and postmodernism and postmodern writers is better understood when viewed from the perspective of the metatexuality of their works and the way they bring the materiality of the medium to the foreground. In this way, the modernism which Bolter and Joyce had identified as the tradition that hypertext fiction follows, becomes closer to the high-modernism/early postmodernism which Kaufmann and others have discussed.

This kind of genealogy, like many other works, will have some overlaps with the other scholarly works in this field especially Glazier's work, Digital Poetics: The Making of E-Poetries (2002). Glazier's study is "an investigation into the materiality of electronic writing," and "addresses, to varying depths, the three principal forms of electronic textuality, hypertext, visual/kinetic text, and works in programmable media" (6). According to Glazier, his book "investigates how the materiality of electronic writing has changed the idea of writing itself" (ibid.). However as the subtitle of his book, The Making of E-Poetries, shows Glazier's discussion focuses on poetry not fiction. Another difference between Glazier's work and this thesis is that the former focuses on the materiality of the World Wide Web, which will be studied in the next chapter, and does not follow a direct line from print into the early developments of hypertext technology and then the Internet. In this chapter in order to show how the tradition of metatextual print works is continued in the electronic medium, the materiality of hypertext fiction will be analyzed.

\section{Materiality of Hypertext Fiction}

What is the materiality of the digital medium and what does it include? A detailed answer to this question helps us to understand how and in what ways the writers of hypertext fiction make it a part of their novels and how digital materiality is different from print materiality. 
According to Kirschenbaum "The fact that you can't reach out and touch software (only the shrinkwrap)" is haptic fallacy. Commenting on electronic textuality, Gass embraces this fallacy, so let's have another look at Gass's sentence:

Words on a screen have visual qualities, to be sure, and these darkly limn their shape, but they have no materiality, they are only shadows, and when the light shifts they'll be gone. Off the screen they do not exist as words. They do not wait to be reseen, reread; they only wait to be remade, relit. I cannot carry them beneath a tree or onto a side porch; I cannot argue in their margins. (A Temple of Texts 165) ${ }^{12}$

Here Gass rejects the materiality of the digital words because they disappear as soon as the monitor is turned off and (at least at the time of writing this essay in the late 1990s), they disappear into the ether. The contrast that Gass is making is between the fixity of words as objects inscribed on a graphic surface and the ephemerality of words represented on a computer screen. The fixed nature of words on paper is the quality which makes Gass appreciate them and prefer them over the words in digital media. Gass's example shows how complex and problematic the issue of electronic materiality is and even a writer/critic who is well-informed about the materiality of print, can endorse the mistaken view/fixture which according to Drucker exists in the popular imagination about the electronic media which discredits it as "immaterial:"

Textual studies have brought attention to the ways various aspects of materiality (type, format, paper, book structure) participate in the production of meaning, just as the 'immaterial' text of electronic environment has become the fixture within the popular imagination." ("Intimations of Immateriality" 152)

However as will be seen, despite Gass's strong opposition to electronic textuality, his concept of transliteracy in many ways anticipates Hayles' concept of materiality and media-specific analysis. Gass's transliteracy refers to the novelist's

\footnotetext{
${ }^{12}$ Arrival of new digital products and Gass's further familiarity with the Internet have now in early 2010s softened his views. It is possible to take an iPad "beneath a tree or onto a porch" and even "argue in its margins." These could be the main reasons that in 2012 he published a work which can only be read on an iPad. C.f. Abstractions Have Arrived (2012).
} 
taking advantage of all the possibilities of the print medium, some of which are listed by Drucker in the quotation above. By treating the book and language as the media in which his work is presented, he invites novelists to explore the possibilities which that medium provides for them. Hayles breaks down the components of the materiality of a work and in doing that makes the readers see the way physical structure and signifying strategies shape the materiality of the text through their interplay.

However, Hayles' understanding of the materiality of the electronic media is different from Gass's in a crucial respect and she coins the phrase "flickering signifiers" to explain how the materiality of the digital medium should be understood. She distinguishes between material modes of information technology, such as books and newspapers, and newer forms of electronic technology. When it comes to reading a print book, the signifier and the signified have a simple relation because the words have a fixed material presence on the page of the print book. The book itself can be stored and handed down to future generations and the words in the book, either created by a typewriter or hand-written will remain there. But the relationship between signifier and signified is not as simple as this when it comes to electronic texts. In these texts, according to Hayles "no simple one-to-one correspondence exists between signifier/signified" ("Virtual Bodies and Flickering Signifiers" 71). A single key on the keyboard can perform several other functions and one single command can wipe out a whole library. An informative example that Hayles uses here is when a book is burned, its text is completely gone, but the lost or corrupted data (i.e. information) on the hard drive (as we had seen in Kirschenbaum's example as well) can sometimes be recovered by the special software which have been specifically designed for this end. So electronic data for Hayles is always "in transit" and can undergo several changes during these transitions. As Hayles discusses, "in informatics, the signifier can no longer be understood as a single marker, for example, an ink mark on a page. Rather, it exists as a flexible chain of markers bound together by the arbitrary relations specified by relevant codes" (ibid. 77).

The codes and how different they are from data, conventions and modes will be discussed in the next chapter, but what stands out in Hayles' quotation here is that the signifiers in the virtual body of the computer flicker, and do not have a permanent position as the letters in a print book have. But this flickering is not a negative 
attribute of the signs on the computer and - as Hayles later discusses in her book-is part of a paradigm shift from the one which focuses on presence/absence to one which is based upon pattern/chaos. This latter pair, can coexist within a system and therefore is not a binary pair in which one is preferred over the other. Such a shift has important complications for literature as well:

The displacement of presence/absence hints at how central pattern/randomness may be in informing contemporary ideas of language, narrative, and subjectivity. The new technologies of virtual reality illustrate the kind of phenomena that foreground pattern and randomness and make presence and absence seem irrelevant. (ibid. 72)

When we look at the materiality of the electronic media from this perspective, the absence/shadow status of the sign on the screen does not qualify as a negative trait, but a different aspect specific to the materiality of the new medium. Although Hayles' is not addressing Gass in her essay, she foregrounds what Gass's main concern with materiality of the print medium has been:

... the literary corpus is at once a physical object and a space of representation, a body and a message. Because they have bodies, books and people have something to lose if they are regarded solely as informational patterns, namely the resistant materiality that has traditionally marked the experience of reading no less than it has marked the experience of living as embodied creatures. (ibid. 73)

In this quotation Hayles explains that works of literature have this dual role of both being a "physical object" and a "space of representation" just as Gass had wanted the readers/writers see the physical body of the book and had condemned the writers who had merely used this body and space as a vehicle for communicating informational patterns. However, Gass himself by denying the presence of a physical body for the works of hypertext makes them merely informational pattern as well. Whereas looking at these works from the perspective of Hayles, helps us see what is distinctive about materiality and how they have taken advantage of it. 
The main reason that electronic media do not meet Gass's requirements for a text as a physical object is that an electronic text for Gass is not "self-contained" and a reader does not have the ability to do what she wants with it. Gass had earlier said that he cannot take it to the bed and in order to read a work of digital fiction one has to go to the desk on which the computer has been placed. But when contemporary technology creates digital objects such as iPad which are self-contained, Gass changes his ideas about them and he even goes so far as creating a work which can only be read on an iPad. So here, portability, paves the way for this new understanding toward electronic materiality.

But regardless of whatever the future updates to the contemporary digital media are going to involve, Hayles' theory provides us with a useful and practical approach to analyze the new media, because it requires us to look deeper into the materiality of digital texts to see whether it is possible to differentiate between different aspects of electronic materiality. This is what Kirschenbaum has done by dividing the electronic materiality into two kinds.

\section{Forensic and Formal Materiality}

Matthew G. Kirschenbaum in his book Mechanisms; New Media and Forensic Imagination (2008) starts his discussion about the materiality of the electronic media by asking this question "what does the materiality of the electronic text consist of?" As the first step in answering this question he divides the materiality of the digital media into two kinds: forensic materiality and formal materiality. Forensic materiality, according to Kirschenbaum:

... rests upon the principle of individualization (basic to modern forensic science and criminalistics), the idea that no two things in the physical world are ever exactly alike. If we are able to look closely enough....we will see that this extends even to the micro-sized residue of digital inscription, where individual bit representations deposit discreet legible trails. Less exotically perhaps, we find forensic materiality revealed in the amazing variety of surfaces, substrates sealants, and other materiél that have been used over the years as computational storages media. (11) 
As can be seen from the above quotation, for Kirschenabum, forensic materiality refers to all the physical elements (both microscopic and macroscopic) used in the computer. In order to show how this materiality is different from the materiality of other non-digital objects, he emphasizes that trace on the hard disk drive is stable and to support this claim, he brings evidence from the stories related to the data recovered from "deleted" drives sold on eBay to those hard drives found and completely restored from the ground zero site at the World Trade Centre. As Kirschenbaum points out, forensic materiality is the kind of materiality which Hayles has studied in her works, but what is missing from her definition, is "the computationally specific phenomenon of formal materiality, the simulation or modeling of materiality via programmed software" (9) (italics mine).

In order to address this issue, Kirschenbaum coins another term, formal materiality, which becomes useful later in this chapter when afternoon and its materiality are analyzed. Here he defines this kind of materiality: "formal materiality thus follows as the name I give to the imposition of multiple relational computational states on a data set or digital project" (12).

So it can be said that formal materiality affects the form of the files and not the substance of the computer. In other words, formal materiality is an emphasis on the manipulation of symbols not the matter. ${ }^{13}$ It is experienced as buttons on the screen or a blank page that is filled by users while writing. These activities create a diversity of relations between applications and the operating system, and lead to material exchanges which entail both inscription and erasure on the hard disk drive. Therefore when you type and save a file in the Microsoft Word software, it does not affect the forensic materiality of your computer and the shape, weight and all the other microscopic or macroscopic parts of your computer stay the same, but this file creates a simulation on the hard disk drive that makes it larger than a blank Microsoft Word file.

Formally, you might erase something on the computer screen, like files in the Trash Can. The file, however, forensically may remain unchanged on the hard drive

\footnotetext{
${ }^{13}$ Kirschenbaum compares formal materiality to bits and forensic materiality to atoms. "Unlike an atom, which has mass, a bit "no color, size, or weight, and it can travel at the speed of light"' (ibid. 11).
} 
as a physical object. Physical erasure takes place at "a variable granularity of the trace, the inscription." So, it can be said that formal materiality is a unique part of the materiality of the computer which is active at the level of symbols and the changes you make to them.

\section{Generations of hypertext fiction}

As seen above, the physical resources of electronic texts offer us a rich variety when analyzed with the proper means and tools. Also both the forensic and formal materiality of electronic media should be taken into account when it comes to analysis of electronic textuality. Now it is possible to divide the field of electronic works of fiction according to the way they differ in their materiality. The history of hypertext fiction has been documented elsewhere (Murray, 1997; Bolter, 2001; Hayles, 2008) and what this research aims to do is revisit the history of this genre in similar fashion to Hayles in Electronic Literature and Writing Machines (2002). This research differs from Hayles' in the sense that Hayles turns to Artist Books in her genealogy rather than metatextual novels.

Although hypertext fiction is a relatively new form, there is an abundance of taxonomies and nomenclatures developed to address it in scholarly books and essays. In this thesis "hypertext fiction" and "web-fiction" will be used for the works which have been called first wave, and second wave, classic and postmodern, and first generation and second generation by other critics. The justification for this taxonomy is that critics such as Harpold (2009) who have used first and second wave have not specifically marked their differentiation. My categorization here becomes closer to David Ciccoricco (2007) who has kept the first and second wave categorization, and has defined the second wave as "works produced for the World Wide Web." This new terminology keeps the name hypertext fiction for stand-alone, script-based works which even in their latest edition need to be installed on computers in order to be read. The names "classic" and "postmodern" hypertexts which have been used by Hayles (2008), as demonstrated by Harpold (2009) include an inherent value judgment about what hypertext has been and has aimed to be. Hayles, who in 2002 had called the early works of hypertext fiction "first generation" and the later ones "second generation" also modified her terminology in her book Electronic Literature to "avoid 
the implication that first generation works are somehow superseded by later aesthetics." Web-fiction which is the term that Joyce has used for his novel Twelve Blue (1996), published on the World Wide Web, has this advantage over the other terms that it directly refers to the World Wide Web as the medium in which stories are written and supposed to be read. The difference that the networked structure of the Web makes, will be the topic of the next chapter.

\section{Materiality of the Medium in Hypertext Fiction}

Hypertext fiction as it is defined here refers to stand-alone, script-based mono-modal works which even in their latest edition need to be installed on computers in order to be read. The main feature of these works is that they only use the modality of written language and are mono-modal in this sense. These works use minimal graphics, with almost no sound or music. The majority of such works have been created by Storyspace ${ }^{14}$ in the early 1990s and published through the Eastgate Systems which is unsurprisingly the official developer and seller of Storyspace. These works are composed of "units of reading" or lexias, and these lexias are connected to each other through links which according to Ensslin "opens up to the reader various navigational possibilities" (20).

The links will be discussed in more details later in this chapter, but the way these links help to generate recurring patterns within works of hypertext fiction is the subject of Bernstein's essay "Patterns of Hypertext" (1998). In that essay he identifies several patterns for both literary and non-literary hypertexts. Although this essay has been cited over 200 times, few of the terms that he has coined for the study of these patterns have become popular with both critics and scholars and two of those words will be explained and discussed here as well. Nevertheless, Bernstein's essay remains an important exploration into the nature of the links and the different patterns that they help to create in works of hypertext.

The stand-alone feature of the works of hypertext fiction means that their material support needs to be updated anytime that a new technology or the upgrades

\footnotetext{
${ }^{14}$ Other programs or writing works of hypertext fiction have been NoteCards, HyperCard, Intermedia, and GUIDE.
} 
to the previous ones change the electronic media ecology. This has already happened once when Storyspace 1 was upgrade to Storyspace 2, which according to Bernstein constitutes "a careful recreation of Storyspace in a completely new computing environment" (172). The current Storyspace available at the website of Eastgate System (late 2013) is Storyspace 2.5 for Mac OS X, and Storyspace 2.0 for Windows.

Studying the works which have been published through the Eastgate Systems shows that the earlier works have been more similar to the works of print fiction than the later editions of the same works. The later editions, as we will see in the comparison between the first and the last commercial releases of the same novel, keep the main two features, being stand-alone and script-based intact, but differ in crucial ways in the way they are presented. Among other things, this difference is a result of the developments in the later editions of Storyspace.

\section{Remediation and Links}

Central to the understanding of how writers employed the potentials of computer generated text and the extent to which print remained a model for them, is the concept of remediation which is - as Bolter and Grusin discuss - at work whenever a new medium arrives and challenges the older medium. Remediation as its name implies is "how a medium refashions its predecessors and other contemporary media" (Bolter and Grusin 19) In order to achieve this goal, the medium presents itself in a way which shows its similarities to the older medium and at the same time focuses on its more distinctive (and usually improved) capabilities. In other words, the medium is initially adapted to the characteristics that are employed in the same way as a preceding one. Like the other examples which Bolter and Grusin have offered in their book, hypertext fiction in its mono-modality and minimizing the graphic devices which it employed, attempted to present its form in a way which does not draw attention to itself because the writers did not want to alienate the readers of print fiction by presenting them with a totally new offering that still was in its formative years. This remediation was so extreme in some works such as afternoon that even the links which directed the reader to the other pages were hidden so that the graphic surface looks quite similar to the pages of a traditional novel. 
On the other hand, there were very few lexias which required scrolling down to read the rest of the lexia so that the flow of the reading is not broken by the interference of the medium in the act of reading. Meanwhile the possibility to click on the Yes/No icon made it easier for a novice reader to find a default reading path in the early works such as afternoon so that a reader's initial encounters with the medium would not be too disorienting. However despite all this, as is expected from a new medium, it offered its own challenging set of capabilities which distinguish it from the older medium. For the works of hypertext fiction, the most innovative feature, which highly differentiated the works presented through it from the works of the print medium, was links. Bernstein highlights their importance:

What matters most in electronic writing is the link. Links are the first new punctuation mark to appear in centuries, the most important technical innovation in our literary machinery since the comma. Other aspects of electronic writing change print practice at the margin ... but links change writing itself. (Reading Hypertext 4)

What makes links so unique and make them "change writing" as Bernstein puts it is that this structural feature of hypertext fiction as Ensslin aptly states "are not only elements of the text, but constitute it." Therefore since the reader does not have instant access to all the parts of the novel in the same way that a reader of print book has, the only element which helps her retrieve another section of the novel is the links, and this is why some critics such as Harpold (1991) have stated that links both bridge and maintain separation between lexias. What this means for writers is that the links provide them with an opportunity for presenting their work in multi-sequential or multi-path order and in this way maintain their kinship with metatextual novels before them such as Willie Masters' Lonesome Wife, and Hopscotch (1966). Moulthrop correctly identifies the equivalent of links in print by saying that "links act like dynamic footnotes," and as we will see later this is one of the features that digital-era metatextual print novelists such as Danielewski take advantage of in creating multi-path novels in print.

From this perspective, links make it easier for a single novel like afternoon, to have several narrative discourses as well as several stories which exist side by side 
and none of them can claim to be "the story" of afternoon. So this organizational feature provides several opportunities for narrative chunks to be arranged in different ways. David Ciccoricco distinguishes three kinds of "hypertext documents" based on the way they have employed the links.

Axial denotes a structure situated along an axis; arborescent denotes a branching structure resembling a tree; and networked denotes an interconnected system of nodes in which there is no dominant axis of orientation. These categories have been applied to hypertext systems but more often with axial and arborescent grouped together as "hierarchical" since both have a main axis. (Reading Network Fiction 5)

This organizing feature of the links and their emphasis on the spatial arrangement motivated the early theorists to analyze them in works like David Bolter's Writing Space (1991), in which he talks about the computer as "a new surface for recording and presenting text together with new techniques for organizing our writing. In other words, it offers us a new writing space" (10). However, in the later discussions on the importance of the links (Aarseth's Cybertext), the connectional feature of the links replaced the focus on their spatiality. In other words, the emphasis was shifted from topography, to topology, and connection replaces location. Therefore, spatial arrangement becomes less important than how the links are connected together and whether the final work is a network or hierarchical hypertext.

In hypertext fiction narrative unfolds through the lexias which have various sorts of connections to each other (as we will see later in the Map View section), so the writer has to pay more attention toward the relations (links) that the lexias have toward each other. Here the chronology would be a direct result (not the cause) of these connections, whereas the arrangement of the chunks of narrative in print fiction follows the chronological arrangements (and its variations such as flashback) rather than topological organization. In other words narrative discourses for each story in a work of hypertext fiction are governed by topology rather than the way it moves forth (or back) in time. 
This is one of the major aspects in which the hypertext by attending to its materiality continues the trend of metatextual print fiction. The links and their relationship to each other within the text required a topological organization which was made possible by the inclusion of maps in the Storyspace, which along with guard-fields are two of its most distinctive features.

\section{Storyspace}

Storyspace is defined as a "writing environment" by Mark Bernstein in his paper on the design of the software "Storyspace 1," which has examined Storyspace from a design perspective and in this regard mirrors the way the designer of Willie Masters' Lonesome Wife, had commented on the design aspects of that book. Storyspace was developed through the cooperation of Bolter, Joyce and John B. Smith and presented for the first time in 1987 at the ACM workshop. ${ }^{15}$ Since Storyspace has been the hypertext authoring program, the material effect of it on writing the novels which used this software to come into existence has been a major factor in how those novels have been written and read. As Hayles says, "so important was software, especially to the early development of the field, that works created in it have come to be known as the 'Storyspace school"' (Hayles, Electronic Literature 6). What should be added to Hayles description is that the Storyspace is more than just one simple writing software such as Microsoft Word, because it provides authors with means of controlling the presentation of their works through the links. These two distinctive features are maps and guard-fields.

\section{Maps}

Map view is one of the possible views in Storyspace (others are Chart view and Outline view), "in which writing spaces appear as nested boxes" (Bolter, Getting Started 17). So as can be seen in the image below, maps are not the illustrations, as their name implies, but the boxes which show the overall relationship between the lexia. Therefore maps include the rectangles which have curved lines with an arrow at the top that connect them together. By clicking on each writing space, it is possible to

\footnotetext{
${ }^{15}$ For a detailed history of the development of Storyspace cf. Kirschenbaum, Mechanisms (176-178).
} 
see which other lexias lie inside that space and what kinds of relations those writing space have toward each other.

The visual aspect of the maps has made them "the preferred interface" (Bernstein, "Storyspace 1"), and "the most powerful and distinctive Storyspace view" (Eastgate Systems Website). Due to their popularity with both writers and readers, the “maps' affordances often shape hypertexts written in Storyspace" (Bernstein, "Storyspace 1" 174). The main characteristic of the maps is that they allow the positioning of each lexia in the backbone of the story and since it is possible to have a variety of linking structures between the writing spaces both internally and externally, the main works written through Storyspace are quite large (afternoon has 539 of lexias and 951 links, Victory Garden has 1000 lexias and 2800 links). The internal links also make it possible to create loops (or cycles) which keep getting repeated and the reader has to close the program and start another reading or move back and forth to find a new unexplored link. 


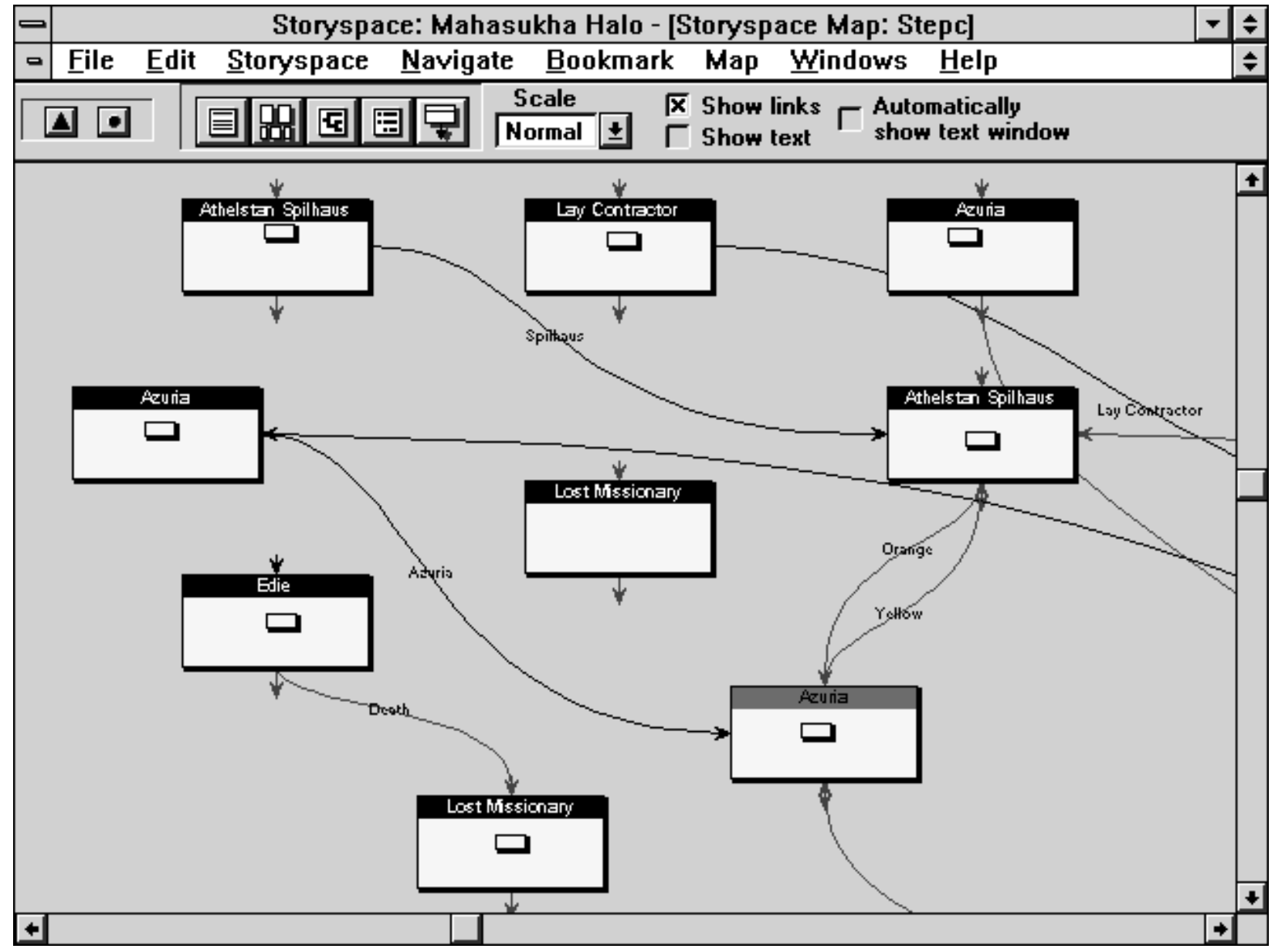

Figure 2.1. Storyspace Map View.

Pictorial arrangement of the maps has been another way which maps have been used in writing works of hypertext fiction. This is what writers such as Timothy Taylor in "LBJ" have done. In Taylor's work, maps are arranged in a way that when zoomed out, they form three crosses positioned next to each other. This use assigns a signification role to the maps which are a physical resource of the text.

What Bernstein defines as Jane's space also becomes an important feature in the spatial organization of the maps. Jane's space-which has taken its name from Jane Yellowlees Douglas's discovery of a lexia in afternoon that was not related to other lexias through links - is the lexia which cannot be retrieved through the linking system and in order to read it, the reader has to open the map view and look for it. According to Bernstein:

Lexias which have text but no links, known as Jane's spaces, might reflect an author's attempts to force reader to use map navigation, as 
well as, authorial deletions, personal notes, messages to critics or collaborators, or mistakes. Jane's spaces are, at any rate, remarkably common; in our sample of twenty eight hypertexts, only twelve lacked at least one Jane's space. (“Storyspace 1" 176)

Jane spaces in this sense shows a unique characteristic of Storyspace; that it has the capability to create lexias that cannot be accessed through the links, but require an alternative method of retrieval. Therefore, these space can be used by writers to various effects for instance "to force readers use map navigation" or leave "personal notes, messages to critics or collaborators." What this means in terms of material affordances of this new medium is that offers a secret place to the writers so that they can leave messages for people who fully engage with the materiality of their work. As will be seen in chapter four, Danielewski does the same in his print novel, House of Leaves (2000).

Early editions of the novel published through Eastgate Systems (especially afternoon) strongly deny the user's access to these maps in order to keep up their print-like appearance. However, these maps appear in the later editions of these novels and step by step distance themselves from their print ancestors. One of the main reasons of the popularity of these maps with the readers is that the maps offer them a visual outline of what lies before them so that there is less opportunity for them to get disoriented or lost in hypertext.

\section{Guard-fields}

Guard-fields are defined as "Boolean expressions based on the reader's selection and previous trajectory" by Bernstein ("Storyspace1" 173), and this feature of Storyspace gives control to the writer to determine which lexias should be visited first and how to limit the access of the reader to the lexias. According to Joyce, guard fields "let a writer set conditions which shape the reading according to simple rules which match the reader's experience of the text against the possibilities it opens to her" (qtd. in Bernstein and Greco 326). In this sense guard-fields limit the reader's freedom rather than enhancing it (as hypertext was expected to do in the early stages of its development), and it is not surprising that they are one of the easiest targets for Aarseth when he discusses restrictions placed on the reader's freedom in hypertext 
fiction in his book, Cybertext (1997). As Aarseth argues, guard-fields grant the authors an unprecedented means to keep certain parts of the text from the reader's access. Therefore, the role of guard-fields can be interpreted as an effort in remediation of the experimental novels such as Willie Masters' Lonesome Wife and Hopscotch which offer multiple ways of reading to the readers, but each one of these readings has been already carefully designed in advance by the writer.

In order to achieve the aim that they have been designed for, guard-fields track the reader's progress in the text and if she fails to visit certain lexias, her reading ends up in a cycle. Bernstein divides the cycles into two groups: Joyce's Cycle and Douglas's Cycle. In Joyce's Cycle the reader "rejoins a previously-visited part of the hypertext and continues along a previously-traversed trajectory through one or more spaces before the cycle is broken" (Bernstein, "Patterns of Hypertext" 22). So when the reader finds herself in such a cycle, she has to repeat the reading path that she has already finished, and find a previously-unvisited link in order to break the cycle, but not all cycles are meant to be broken and if the reader finds herself in such a cycle, which Bernstein names Douglas's cycle, it means the "end of a section or the exhaustion of the hypertext" (ibid.). These cycles create recurrence which, as Ciccoricco (2012) shows, makes close reading of works which have a large number of lexia possible.

\section{Narrative in the Hypertext Fiction}

If the medium has an active role in shaping the content of a work, then our current definitions of the story may be regarded as the result of thinking through the medium of print. Hence, if the book, as the quotation from Joyce demonstrates at the beginning of this chapter, is "merely a fleeting, momentarily marketable, physical instantiation of the network" which can be arranged in several other ways, as soon as a new medium enters the scene, these definitions will change too. From Aristotle's tripartite definition of a plot, to the countless variations of this model, thousands of novels have been published, most of which have exemplified Aristotle's definition for their narrative structure. Meanwhile, even within the medium of print, experimental novelists like Gass have shown that several other ways for constructing narrative are possible and in doing that have tried to push the boundaries of the genre. 
For Gass the novel was a "pool of stories" or "cataract of narratives" which the author has constructed and the medium has made possible. This kind of conception of the novel is closer to how writers as early as Sterne had conceived of it. No story is ever complete and the narrative always leaves certain parts of the lives of its characters in the dark.

Hypertext fiction, by not providing closure and creating alternative reading paths for the embedded stories, provides a different range of opportunities for the narrative. Bolter and Landow in the early phase of the development of hypertext fiction argued that links are the distinctive feature of hypertext fiction, Abbott supports their arguments by saying that the difference that hypertext makes to narrative "depends not on the mere presence of hypertext linking, but on how that linking is deployed" (Abbott 33). In fact links, as Aarseth has shown in his book, can be quite restrictive and conceal several parts of the story from the reader-as we already have seen in the case of guard-fields-whereas the reader has access to all parts of the book when reading a work of print fiction. Therefore, the way the author employs these links has a lot to do with what the final outcome will be and whether that work can be considered a work of narrative or not. Abbott provides us with a simple but useful criterion for this: "That they [links] permit readers to some degree to arrange the narrative discourse, and to distribute its parts differently in different readings, does not in itself violate the essential narrative condition, as long as there is a story to be recovered" (Abbott 34) (emphasis in original). So one of the main features which will be studied in respect to the narrative (and will be analyzed in the narrative section of each case study) is how the story is recovered in each work and how the materiality of each section affects the narrative and the ways it can be recovered.

Bolter and Joyce highlight a narrative-related aspect of the hypertext fiction which they refer to as "temporal character" of hypertext fiction. What this temporal character entails is the manipulation of story time ${ }^{16}$ or plot:

\footnotetext{
${ }^{16}$ Abbott uses the phrase "the time of the narrative discourse" to refer to the same concept, which is the time that it takes for a reader to read a work of fiction.
} 
In printed fiction the author is free to manipulate the time in which his story takes place, and every good author does so. However, the plot, the author's manipulation of story time, is itself static. Printed fiction is one-dimensional in the sense that we need only one dimension in order to represent the experience of reading it. The episodes (chapters, sections, cantos, books, volumes) are realized through time as we read. The links between episodes are fixed in the course of writing, and the reader has no obvious or effective way to alter the order of reading. In electronic fiction multiple links among episodes allow our temporal experience of the plot itself to vary. Time may be fluid in a printed novel, but the representation of time is fixed, as the fixed pages of the book mark the progressive stages of the narrative (Bolter and Joyce 47).

While a reader is free not to observe the print order and to skip sections, read the last chapter first, and so on, this does not alter the fact that all the elements making up a conventional print novel's narrative are presented in a single, fixed sequence. The distinction between plot and story made by Bolter and Joyce is similar to Abbott's distinction between story and narrative discourse. For both of them there is a story which is being narrated by the author through the text, but the way it is presented to the reader through the medium creates the plot (or narrative discourse in Abbott's terms). In the above quotation Bolter and Joyce emphasize the way the narrative discourse is changed through its engagement with the medium in which it is presented.

One useful way to describe the progression of narrative in the works of hypertext fiction is recursive. As Bernstein says: "Hypertext structure is perceived through recurrence. The cycle, not the branch, goto, or jump, is the central hypertext structure" (Bernstein, Reading Hypertext 8). What this means for the narrative is that, as we saw in the case of the guard-fields, certain parts of the narrative are repeated again and again in a reading and these recurrences or re-visits, as Douglas (2001) has shown in the case of afternoon, move the narrative in different directions. 
The appearance of a Douglas Cycle in course of narrative can imply the ending of one narrative line. So the reader has to either go back and start another reading or close down and restart a new reading. But an important contribution of the cycles (Joyce's Cycles in particular) is that the lexias which have been visited and read in the past now find a different meaning through a new reading which is also a new narrative discourse. One of the frequently quoted examples of this feature is the opening lines of afternoon which describe a cold setting in a poetic way:

By five the sun sets and the afternoon melt freezes again across the blacktop into crystal octopi and palms of ice-rivers and continents beset by fear and we walk out to the car, the snow moaning beneath our boots ... (begin)

Upon revisiting, as Bernstein (1998) suggests, this scene might be describing the "wreck of his ex-wife's car" and "rivers and continents be beset by fear" and moaning of the snow can be "the wreck left after the car (and the bodies) have been removed."

It goes without saying that hypertext structure by itself does not create networked patterns. As Aarseth demonstrates in his book, a work of hypertext can be unicursal, that is, "move in a continuous line from start to finish (Hayles, "The Transformation of Narrative" 22) or it can be multicursal, which means "different branching points may be chosen, so that multiple pathways potentially exist" (ibid.). So the possibility of having different reading paths in a work of hypertext fiction is one of the major features of hypertext which later experimental print novelists such as Danielewski take advantage of in their works by sending the reader back and forth through the footnotes, endnotes, appendices, etc. and in this way create alternative potential reading paths. The multicursal narrative, which Aarseth in a later essay comes to call multi-path narrative, can be found in other media such as video/film and metatextual print novels like Willie Masters' Lonesome Wife as well. As we have seen in Gass's novel, linearity as a conception of the order of reading breaks down in those sections which have three or more parallel narratives. In that work the reader might go to read in a linear fashion, but then realizes that narratives are divided into three or more sets and are dispersed over the pages in those sections, so she has to stop her 
linear reading and reads one set and then the other set, and by doing that she brings in the element of recursion, which means moving from the narrative discourse as the order of reading to a kind of variable order of narrative discourses which anticipates the link.

But still the central question remains: how can narrative, which according to Ryan in her book Narrative Across Media: The Languages of Storytelling (2004), is "a way of organizing experience that follows a rigorous internal logic, makes sequence supremely significant, and strives toward closure" (331), exist in a hypertext system? She examines this question by considering the accounts of proponents of narrative in hypertext fiction such as Hayles, whom she quotes as proposing that "in electronic fiction, narrative takes shape as a network of possibilities rather than as a preset sequence of events" (330). Ryan discusses the features of hypertext fiction and analyzes the possible narratives in afternoon, she goes on to conclude that:

Many scholars ... have indeed observed that hypertext is not a good medium for the creation of compelling plots that live from suspense and emotional participation in the fate of characters .... It promotes a metafictional stance, at the expense of immersion in the fictional world. This explains in part why so many literary hypertexts offer a collage of literary theory and narrative fragments (Narrative Across Media 542-543).

Ryan's definition of narrative leads to her claim that "narrative cannot be consummated piecemeal." Whereas as we had seen with Gass, the narrative is always made of chunks which could be dispersed in different parts of the page, or in the case of hypertext fiction located in several internally linked lexia. ${ }^{17}$ An understanding of the way the links and the cycles confirm or change the narrative strands fits better with hypertext fiction because it pays particular attention to the way its materiality has affected the way the narrative is made within it. So here chunks which create lexias become a defining characteristic of hypertext and the way it is employed in

\footnotetext{
${ }^{17}$ Another point of departure for this thesis from Ryan's discussion is that experimental novels try to move against expectations, pushing the boundaries of the medium further and using it in non-conventional ways, whereas a major implication of Ryan's line of inquiry is establishing and adhering to certain specific conventions.
} 
experimental print novels as well. Therefore, the "chunking-linking" technique of hypertext, as Hayles puts it, does not lead to what Ryan calls a "jigsaw puzzle mode of reading," but provides us with a possibility of reading which is not as open-ended as the early theorists had claimed, but it is not as restrictive as Ryan has discussed.

\section{The Reader in Works of Hypertext Fiction}

The reader of a work of hypertext fiction, just like the reader of a metatextual print novel, needs to pay attention to the way the physical resources of the work interplay with its signifying strategies in order to present the work of fiction. This requires readers submit to the work in order to be open to its meaning and have an (inter)active role in their reading process. Therefore, one of the concepts which has been discussed at length in studies of hypertext fiction is the concept of the reader and whether he is empowered or not. One of the first proponents of the empowered reader, or the reader-writer (wreader), was George Landow who came up with this term and was later stigmatized for it by other critics such as Aarseth.

What Aarseth proposed in order to distinguish the reader of a hypertext fiction from the reader of a conventional print novel was the "nontrivial effort" required of the former to "traverse the text." The advantage of Aarseth's term lies in the fact that it is applicable to both print and hypertext, and shifts the attention from the power/control relationships to the efforts that the reader has to make, which are more than turning the page. Though Aarseth does not specifically discuss the materiality of the medium in the same way that Hayles does, by bringing in and comparing the works of print with the works of hypertext, he offers a new insight into the way the reader has to behave in reading each text. Aarseth's discussion becomes particularly useful because of the choice of the word "traverse" which means "going across." This word emphasizes the spatial dimension of reading and makes the text a place where the reader does not look beyond it, but goes across it.

However, even for works of hypertext fiction, each reading becomes "rereading" because, after the reader has finished reading one lexia, she has to move her eyes across all the words in that lexia to find the word which drew her attention to click on them and by doing that parallels the spectators of the statue that Gass had described in the previous chapter. Both spectator and reader look at the work of art 
and move their eyes across it, following its form but without a defined end point. In the case of the reader of hypertext, she has to make a decision about which word in any lexia to click on, and in this sense each hypertext is comparable to Gass's metaphorical statue. 


\section{Case Study 2.1. afternoon, a story ${ }^{18}$}

As both Kirschenbaum and Harpold have demonstrated, there are several afternoons. Just as during the last twenty years the medium of the computer and its operating systems were developed further and further by their manufacturing companies, so did Joyce (and his publisher) develop afternoon. Harpold has identified sixteen versions for afternoon up to 2007, six for Macintosh computers and six for Windows, two translations (into German and Italian), a web porting, and a print version. The last two are only a selection of the lexias (fifteen lexias for the web version, and ten for the print copy of the Norton Anthology of Postmodern American Fiction). For the contemporary reader in late 2013, apart from the web ported version only the sixth edition (for both Mac and Windows users) is commercially available, and the previous editions are already collectors' items. This research analyzes the third edition of afternoon, which is the first commercial edition of the novel. Although afternoon as the first hypertext novel has primary importance for this research, its history, development and the myriad of changes that it has undergone will not be studied here. ${ }^{19}$ Instead, this chapter will discuss the metatextual features of afternoon, and it will demonstrate how all these features are dependent on and a function of the interplay between this novel's physical characteristics and its signifying strategies.

The materiality of afternoon should be studied in at least three ways, two of which are forensic and one of which is formal materiality. The first kind of forensic materiality of this novel refers to the data storage medium (3.5 inch floppy disks) on which it was sold, and the second kind of forensic materiality refers to the machine which retrieves information from the storage medium and makes reading afternoon possible (a Mac LC here). The formal materiality of afternoon refers to the software (Storyspace) by which this novel has been written and presented to the audience. All these three at the same time provide various opportunities and create several limitations for the early works of hypertext fiction writers. In addition to all these,

\footnotetext{
18 This thesis follows Joyce's original referring to afternoon without capitalization.

19 Terry Harpold (2009) and Mathew Kirschenbaum (2008) have written insightful chapters on these issues in their book. Reading their books can be very informative for readers who are interested to explore these fields.
} 
packaging and the way the floppy diskettes have been sold by their publisher (Eastgate Systems) creates another level of materiality which will be discussed below. Michael Joyce who was one of the developers of Storyspace states that it took him four years to figure out how to make it a useful tool in writing novels.

Yet a fairly common reaction to hypertext and hypermedia systems in product reviews, in technical literature, and among everyday users of these tools is the one expressed by Jeffrey Conklin in his still definitive 1987 article, "One must work in current hypertext environments for a while for the collection of features to coalesce in a useful tool." This is a kind way to say that you have to figure out what to do with these things. I have spent much of the last four years figuring out exactly that. As a code-developer of Storyspace, I have approached what to do with the things as a design question; as a fiction writer seeking to work in a new medium, I have approached it as an artistic question, and, as a teacher, a practical, pedagogic, and sometimes a political one (Of Two Minds 39).

Thus, it can be said that many of the features of afternoon had been already thought about by Joyce, and he created that novel with a comprehensive knowledge of the capabilities of the medium and both layers of its materiality, the computer and its new range of abilities (which required its users to have a certain degree of computer literacy to be able to use it) and Storyspace as the authoring tool for afternoon. This puts Joyce in sharp contrast to Gass who was not happy with the published novel and believed that what he had been "trying out" had not worked. A major part of Gass's discontent was due to the fact that his "ability to manipulate the spatial and visual side of the medium" was not sophisticated enough to make the "visual business... successful."

Joyce, on the other hand, did not have to worry about any of these problems because during those years he had thought about the issues of software design and functionality raised by writing fiction in a digital medium, and how to add those features (such as guard fields) which had seemed essential for him in writing a novel on the computer. On the other hand, afternoon did not have "to go through so many 
hands" because, in writing it, Joyce used the capabilities of the authoring tool which he had co-developed and it can be said that he had complete control over the work from start to finish. Thus studying afternoon, and comparing it with other novels such as Willie Masters' Lonesome Wife, shows us how and why Joyce has responded to the choices that any writer has to make when he uses a totally new medium (with new set of capabilities) to write fiction.

\section{Physical Organization and Design}

Afternoon is the first work of hypertext fiction, and it is one of the most discussed works of hypertext fiction. From Landow (1994) to Bell (2010) many scholars have discussed various features of this work (closure and possible stories (Douglas, 2001), freedom of the reader (Aarseth, 1997), and narrative (Ryan, 2006). However, apart from Kirschenbaum (2008), and Harpold (2009), almost none of the books and essays have discussed its materiality, and how changes in different published versions of afternoon modify the experience of reading this work. What follows is a media-specific analysis of afternoon.

The medium used here (instead of a simulated program) has been an actual Mac LC with forty MB Hard Drive with 6.0 Mac OS and two MB RAM. The CRT monitor for this Mac was a fourteen inch ViewSonic E641 which has a display resolution of 512x384 pixels at eight-bit color. afternoon was published on a three and a half inch floppy disk which had the capacity limit of 720 Kilobytes and during the early 1990s was the most popular portable storage medium for electronic data. This storage medium imposes its own limitations (in addition to the opportunities it provides) for publishing a work of hypertext fiction. The first (and probably the most important) limitation imposed on the authors by this medium was its small storage capacity which discouraged the authors who wanted to sell their works through Eastgate Systems (and probably designers of the software) from creating large-scale works. $^{20}$ This could have been one of the reasons why afternoon, like many other

\footnotetext{
${ }^{20}$ The second release of Storyspace, King of Space (1991) by Susan Smith was published on three 3.5 floppy disks, and Uncle Buddy's Phantom Funhouse(1992) by John McDaid was published on five 3.5 floppy disks (and comes in a box which is totally different from the standard packaging of other Storyspace releases). However, these are the exceptions and most other works released by Eastgate Systems were either published on one or two 3.5 floppy disks.
} 
works which follow it, is mainly script-based and has minimal graphics. The only picture on the opening lexia which shows three characters is small and blurry.

The version of afternoon which is studied here comes in a jacket which, in an obvious attempt to remediate books, functions as the cover for the novel and, similar to its print equivalents, has the name of the novel and the author on the front. A short introduction about Michael Joyce along with a selection from the reviews of other famous novelists and scholars is printed on its back. Inside the cover, in addition to the floppy diskette of afternoon, there is an actual printed booklet. ${ }^{21}$ This eight-page booklet has eight sections: a) Afternoon, a story (with more selections from the reviews), b) Getting Started, c) Reading Afternoon, d) The Tool Bar, e) Questions?, f) About the author, g) License Agreement, h) Limited Warranty and Disclaimer. Sections c and d (in slightly altered forms) are repeated in the electronic version of the story under the lexias of "hypertext" and "read at depth" and precede the story. Sections a, e, f, g, and $\mathrm{h}$ are the standard sections which can be found in almost all contemporary print novels, and adding them to the booklet here is a further attempt at the remediation of the material form of the print novel. It is not clear whether having a printed booklet in packaging has been the decision of Storyspace designers or the publishers (Eastgate Systems), but it clearly aims to further justify Joyce's claim that afternoon is a novel.

One of the main challenges for Joyce, as the first hypertext fiction writer, was finding an electronic equivalent for the page and how to move to other pages of the novel on the computer screen. In order to address this issue Joyce chose to use (hidden) links and have every lexia fit to the screen, so that the novel could partly have a visual similarity with the book. Another issue which has been taken into account in the physical organization and design of afternoon is the bookmarking capability of the novel, which is shown in the program's asking to save the reading path before exiting the program while you open it next time for a new reading. ${ }^{22}$ This

\footnotetext{
21 This booklet hints at strength of the print tradition which exists and leaves its mark and trace on even the works which ironically were hailed by some critics as the nemesis of print

${ }^{22}$ The accompanying booklet of afternoon pinpoints this feature: "Each time you open afternoon, you can choose whether to begin a new reading or resume previous reading. If you resume a previous reading, you begin at the place where you stopped reading previously."
} 
feature allows the machine to automatically trace the reading sequence of every reading and stresses the procedural aspect of the reading. In a print novel, a reader may use a custom-made bookmark or a piece of paper to bookmark his reading path, and after the novel is finished, the reader does not use the bookmark anymore. However, even if you have visited almost all 539 lexias of the story and have made an intensive study of afternoon like Douglas (2001), the machine still asks you whether you want to "save the current place in your reading of afternoon," a request which implicitly notes the never-ending nature of reading this novel. No reading is complete and the physical organization and design of this novel stresses this repeatedly. This bookmarking feature might seem trivial at the beginning, but it is one of the features of the software which has been seriously thought about (and later developed) by the developers of Storyspace. Bookmarking in addition to implying kinship with print novels (which cannot be read in one sitting) also implies the existence of the guard-fields which have been discussed above. You cannot simply pass through the maze of afternoon in one reading. It is a place that you have to return to (probably every afternoon after work) and that is why you need bookmarks to help you explore various corners of it.

afternoon's 2001 edition for Windows and Mac comes in a CD with a glass cover and the booklet which accompanied the first commercial edition has been downsized to a small photo. Moreover, the information on the booklet has been added as separate file which appears in the same folder where afternoon is. There is also a text file in which the copyright information of the afternoon appears. Several other navigation options have been added to this version too. For instance "locate writing space" from the Navigate tab allows the reader to search among the lexias. Apart from all this, the 2001 edition has an important statement by Joyce. Under the Storyspace tab in the software, there are a couple of keywords which differ in Windows and Mac versions. The Keywords in Windows are fragments, moaning, poetry, wall, winter, yesterday, but the Mac version has some additional keywords which include black, blue, cyan, green, magenta, and red. This become even more interesting when we search these colors in the "find text" search option of the novel (under the drop-down Navigate tab), and find out that some of these colors (such as 
cyan, and magenta) have not been mentioned in the novel at all. Cyan, Magenta, Yellow and Black are the basic colors used for printing color images. On the other hand, Red, Blue and Green are used for creating images on the computer screens. The first obvious interpretation of this feature is that, similar to the creation of colors, different stories are created when different lexias of this novel are placed alongside each other. The second interpretation is that since cyan, magenta, and yellow are "subtractive" colors, they get darker as you blend them together, and ultimately create the color black. On the other hand, red, green, and blue produce the color white when they are blended together. Since the only colors used in this novel are black on the white background, what Joyce means here is that the whole range of colors are available in his novel, and his work is a blend of all these colors which have been used for creating images on the computer screen and printing them on paper. What this means in textual practice is that his story incorporates the elements of print and computing media and combines them together to achieve a kind of synthesis (or compromise) between these two; the long tradition of the novel and the new medium of the computer.

\section{Narrative Strategy}

One of the major motivations for Joyce to venture into electronic textuality was writing "a novel that would change in successive readings." After all, as the choice of words for the parts and functions of the computer-Random Access memory, process, and cell- suggests that the computer has been modeled on the brain, then modeling a narrative on the way associative function of the brain would have been the most obvious option for writing a novel using the medium of the computer. One of the popular equivalents of this "associative function" is the psychological plot and stream of consciousness. The narrative of afternoon has this potential of giving the reader the option to choose which character's thought to follow. As soon as the characters of the novel (Peter, Wert, Nausicaa, Lolly, and Lisa) appear on screen you can click on their names to read more about them and their backgrounds. However, this effort does not always lead to new threads of the story, nor does it have the ability to change the focus of the narrative from Peter's story to the other characters. In this respect, afternoon remains Peter's story from start to finish, but Peter's story is not solely his story too, and it intermingles with lots of 
other stories: "I'm not sure that I have a story. And, if I do, I'm not sure that everything isn't my story, or that, whatever is my story, is anything more than pieces of others' stories" $\left(\mathrm{Me}^{*}\right){ }^{23}$

In writing afternoon "Book of Sands", a short story by Jorge Luis Borges, could also have been a major source of inspiration for Joyce:

I cannot say that I have not been waiting to be asked what prompted afternoon, a story or what it seems to me to be as an art object, an electronic construction, a constant occasion, Holzer's chasing light high above the town square of the city of text, nomadic shiftings of momentary truths like sand flitting from the stone shelf in the wind, a story that changes each time you read it_- "a form for what yet does not exist." Thus I am happy to point to what is perhaps too obvious to be seen: that the screen gives (way) before $\&$ with \& after the touch, its surface not so much mottled as smoothed in the "places that yield" (the "words with texture" as the reading "directions" say, themselves nothing less than exhibit notes). What kind of text has directions? The hypnotic, the Eventualists' stimulus framed in the mirrored eye (viz.Lombardo 1979), the shape of the mind later seen in dreams, the text of water where Bridal Veil Falls (Basho's Urami-no-Taki) smoothes the sandstone shelf in one hundred million drumming fingers of light. (Of Two Minds 186)

By using texture and imagining the work in this way, Joyce is trying to remind the readers of the Latin origin of text, texere, which means to weave. So in the same way, a text is created by various threads which come together (are woven together) in every reading and that is why in a lexia titled "in my mind" he says "real interaction, if that is possible, is in pursuit of texture". The "pursuit of texture" is the key phrase here and the textual structure of afternoon (except the default paths, which offer a rudimentary and limited experience of reading that novel (either 27 lexias or 39 lexias [clicking respectively on yes or no] out of 539 lexias can be parsed in that way) is woven a in a way which makes sense to the reader who is in pursuit of texture. This is

${ }^{23}$ The lexias of afternoon are referred to by their title here. 
also why Joyce has observed that the words which "yield" are the "ones which have texture." Another important implication of this weaving (or quibbling as Carolyn Guyer later shows in her hypertext fiction of the same name) is that there no single pattern, and these threads are created as the interplay between a literal interaction between the machine and the reader. Therefore, reading is always a process, a process which involves materiality as the interplay between a text's physical characteristics and its signifying strategies.

Clicking on pronouns, however, makes it easier to understand who the narrator is and to whom s/he is talking. These yielding pronouns make reading afternoon less difficult and help the reader to make sense of what is happening in the story and to choose what lexias to follow next. Here again the machine facilitates the act of reading and gives the reader a new opportunity which he cannot experience in an experimental work of print fiction. The default path which starts with yes (or pressing a "wave of returns") has been best explained and analyzed by Walker, (1999); and Douglas (2001). However, the story through a wave of "nos" has not been fully discussed in the academic discussion of afternoon. This feature (being able to reply "no" to the narrator who asks "do you want to hear about it?" in the first lexia of the story) according to Bolter makes this story different from computer games, which had a lot common with the script-based hypertext novels during this stage. ${ }^{24}$

One of the metatextual features of afternoon appears within a lexia named "with a computer." This lexia which is made of only four letters and lots of ones and zeroes is a self-referential lexia for the media through which this novel is instantiated. Apart from this, Joyce uses the highly popular technique of authorial intrusion in the story and Michael Joyce and another person (maybe his wife) pop up in various parts of the story and talk about the story which is being narrated.

The narrative strategy of afternoon has been compared with the narrative strategy of Cortazar's Hopscotch (1966), because the latter in its table of instructions

\footnotetext{
${ }^{24}$ For a detailed comparison between the script-based games and hypertext novels of this time see Ryan (2005).
} 
invites the readers to choose between two sequences of reading. ${ }^{25}$ Expendable chapters in Hopscotch start from chapter 57 to the actual end of the book and can be ignored in the first sequence of reading, which is named "normal fashion" by the writer. The second sequence starts from chapter 73 and the reader has to follow a certain sequence of chapters to read the rest of the novel. This kind of organization seeks to lessen the medium's influence on the way the story is instantiated by it. However, even a cursory glance at the second sequence of reading demonstrates that the original (linear) arrangement of the story (and ultimately book format) has been kept almost intact, and the expendable chapters have been dispersed through these chapters. For instance the order of the second reading sequence starts like this:

73-1-2-116-3-84-4-71-5-81-74-6-7-8-93-68-9-104-10.....

However, for afternoon there is more than one story, that is, all the events which occur cannot be arranged in one single chronology. Instead, Willie Masters' Lonesome Wife offers a better genealogy for afternoon. There are several threads in the book which can be read in different orders and none of them can claim to be the main story. However, despite this similarity, what distinguishes afternoon from Willie Masters' Lonesome Wife is the ability of the computer medium to generate different sequences for reading afternoon, whereas there are limited sequences of reading for Willie Masters' Lonesome Wife, which is mainly due to the inability of the book medium to generate further reading paths after these sequences have been exhausted.

Another aspect of afternoon which makes it more similar to the works of metatextual print fiction is that Joyce's primary purpose in writing afternoon (and the way he describes it later) was not to offer a reader immersion and agency in that work. Instead he wanted to write a story which would change with every reading:

Immediately, I discovered that what I really wanted to do was something else. What I really wanted to do, I discovered, was not merely to move a paragraph from page 265 to page 7 but to do so

\footnotetext{
${ }^{25}$ This comparison has been reinforced by Joyce's quoting a part of the Author's Note in afternoon: "In its own way, this book consists of many books, but two books above all. The first can be read in normal fashion and it ends with Chapter 56....The second should be read beginning with Chapter 73 and then following the sequence indicated...." (Hopscotch)
} 
almost endlessly. I wanted, quite simply, to write a novel that would change in successive readings and to make those changing versions according to the connections that I had for some time naturally discovered in the process of writing and that I wanted my readers to share. (Of Two Minds 31$)^{26}$

Such an approach to writing fiction implies the writer's interest in experimenting with the form and the material capabilities of the medium and this is exactly what metatextual print writers were doing in the second half of the twentieth century. However, Joyce is somewhat conservative in the choices that he has to make in creating the first novel of its kind: a psychological story narrated by stream of consciousness technique, lexias which fit to the screen, and invisible links have all aimed to reduce the alienating effects of technology in this novel. However, the very fact that there is no ultimate end to the novel and, according to Sarah Sloane, "the mystery of the opening frames is never resolved," (Digital Fictions 124) draws attention to Joyce's concern to make the "suspect quality" of closure manifest and create a novel which has no definitive endings.

\section{Reading Process}

Several people have explained that their first reading of afternoon led to frustration (Walker, 1999; Bernstein 2009). These readers have also described their reading as clicking randomly on some words without paying attention to the way Joyce had asked the readers to read afternoon. In "read at depth" Joyce asks the reader to answer questions by clicking the Yes/No button, and in subsequent readings, to explore the links between screens by choosing the browse button. This seems like inviting the reader to start by the default path and then move on to the more complex reading which involves trying to find the words which yield. However, in the lexias which follows (read at depth), Joyce more directly mentions the default path: "you can answer yes at the beginning and page through on a wave of Returns." The first question in the default path which has a yes/no answer comes in the famous last line

\footnotetext{
${ }^{26}$ He acknowledges the role of the writing program that helped him achieve this aim: "Storyspace, which in the spring of 1987 enabled me to write a hyperfiction, afternoon, a story, that changes every time you read it" (Of Two Minds 32).
} 
of the first lexia of the story, (begin)—“do you want to hear about it?"- -and as it has been observed by several critics including Douglas, answering yes or no takes the readers to two different stories. On the other hand, since answering yes and clicking "on a wave" of returns or yes takes the reader to some meaningful section of the story, this might have been the privileged way for the first reading of afternoon and, as Jill Walker has observed, reading the default path first eases the new reader into reading afternoon. Talking about her own experience, Walker says: "Reading this sequence gave me enough background information to start enjoying the leaps between story lines, and to understand the connection where I'd earlier only been confused" (in Bernstein, Reading Hypertext 22).

In other words, reading the default sequence requires a continuous repetition of a specific simple action (either clicking on the mouse or pressing the Return key), which is practically the digital equivalent of turning the pages. Clicking on the word “yes" (or alternatively "no") after finishing reading every lexia will automatically take the reader to the next lexia which may or may not be directly related to contents of the previous lexia. This experience of reading makes afternoon a linear stream-of-consciousness story about a man who may or may not have seen his son and ex-wife die in a car accident. However, this story (from the pool of other stories), stops at a certain point and it is impossible to go any further than the lexia entitled "I call." This way of reading afternoon (which I suspect might have been the most popular way for the first curious readers of afternoon) has almost all of the features of print fiction, a proper beginning, a coherent sequence, and the only action required of the reader is click on "yes" (or press the return key) to follow the plot and the protagonist's stream of consciousness. Therefore, following the Yes/No default paths results in Douglas's Cycles. The narrative at various other points in time arrives at Joyce's Cycles, but in order to get out of a Douglas's cycle, the reader has necessarily start a new reading.

The second reading requires the reader to click on the words "which have texture, as well as character names and pronouns" (read at depth). In every lexia, most of the words are linked to a single other lexia and regardless of the word selected most of the time the reader is taken to the same lexia. However, there are a few other words which by clicking on them take the reader to a totally different lexia. The important 
thing here is that this linking structure changes when the reader has visited specific lexias which are meant to be read first. Joyce implicitly mentions the mechanism behind this - guard-fields — in his directions for the reading the story: "A word which doesn't yield the first time you read a section may take you elsewhere if you choose it the next time you encounter the section again " (work in progress). Joyce uses the guard-fields "aggressively," and reading is at the mercy of the guard fields, consequently the choices that the writer has made in advance for the reading sequences in the story provide her with what I would like to call "limited freedom" for browsing the novel.

The third way of reading afternoon which can be a combination of the second kind and the first one is clicking on the browse button and following a link from the new window which pops up and. This is the reading which Joyce invites the readers to in their "subsequent readings." This way of reading provides a more purposeful approach to the work, because the reader instead of clicking on a word and hoping it yields to her touch, can see which links are available in every page. 


\section{Metatextual print fiction in the early age of hypertext fiction}

One of the effects of the development and wider usage of computers during the early 1990s was that it brought about a general awareness of the media of communication and how they materialized information. Also due to the significant rise in computer literacy (electracy) (Ulmer, 2003) many writers of print fiction started typing their novels on the computer and gradually the computer replaced the typewriter as the main writing machine for writers. ${ }^{27}$ This new medium of writing presented the writers with new features which facilitated writing, and editing what they had written without being required to type the whole page again. These features affected their writing in various ways.

The move away from typewriters to word processors seems to be having an impact on writing today that some people are comparing to the impact that the printing press, and later the typewriter, began to have on writing practices. For instance, I've noticed that more and more writers are breaking up the linear flow of the narrative by using devices like footnotes and endnotes, glossaries, and other formal methods to deflect the reader's eye from its usual left-to-right, front-of-the-book-to-the-end movement. ... $\mathrm{A}[\mathrm{n}]$ elaborate example would be the elaborate glossaries, endnotes, appendices and so forth that you find in many of William Vollmann's novels. When people wrote books on typewriters, the act of creating a footnote was very laborious, time-consuming work, whereas now, you can almost effortlessly insert footnotes, create glossaries, and even generate indexes. Just the fact that it's now so much easier to create footnotes or other textual "layers" seems to have encouraged writers to think of what they are doing less in terms of developing linear narratives than in presenting works which are "textual assemblages." (McCaffery, "Haunted House" 117)

\footnotetext{
${ }^{27}$ Some writers, on the other hand, "persisted in pecking away at a manual typewriter" (Kurt Vonnegut, 1997) and some of the Luddites such as William Styron and Stephen King preferred to "write their novel with pen on yellow legal paper."
} 
Partly due to the new capabilities of word processors like Microsoft Word, (which had become a commercial success in 1990, and has kept its popularity during the last two decades), and partly due to the writers' interest in experimenting with these new capabilities, some of the metatextual novels published during the early 1990s were trying to show these changes in their format and instantiation. As McHale has shown, some of the postmodernist writers such as Federman, Katz, Brooke-Rose and Gass, by using concrete prose in their works, had already experimented with the traditional page format in the 1970s and 1980s. These works can be considered as the stepping stones towards further experimentations in the book structure and page format. Some of the print writers in the early 1990s started experimenting with the new medium. One of the most significant works of these writers is Agrippa (a book of the dead) (1992) by William Gibson. This poem had been programmed to erase itself after a single use. At the same time, many of the classic novels were ported to the Internet and some digital libraries like Project Gutenberg, which had started in 1971, became more popular and more easily accessible for the public. This changing scene of literacy made the role of medium more visible and though reading novels on the computer screens in the early 1990s was an arduous task, writers of print fiction, book designers and publishers took advantage of the new capabilities of this new medium which were being developed further and further day after day. The computer medium during those days was not as fully developed as it is now, and many of those experimentations were still mostly script-based; until 1995, most of the metatextual works had to confine themselves to experiments in typography and page layout.

\section{A Media-specific Analysis of Fax Messages from a Near Future}

Fax Messages from a Near Future (1994/1996) is novel by Brazilian architect and urbanist, Jorge Wilheim. This novel is about the fax exchanges between Professor $\mathrm{J}$ and Titus who are living 30 years apart and have a diachronous dialogue by sending faxes to each other. This novel follows a linear plot and its format might not seem to be metatextual in the first glance. However, a closer study of this work reveals its metatextual features and demonstrates how this novel, in using the available capabilities of the medium of its production, can be an exemplary novel of its own era. 
Professor $\mathbf{J}$ who is living in 1994 uses a Toshiba fax machine and he suddenly receives faxes from Titus who is living in 2024. Right at the beginning of the novel, there is a statement about how fax machine materializes the information:

After the fax gives its incoming message tone there's usually a pause while you wait as the message retreats and peeks out and leaves you there holding your hand out. A pause of mounting suspense, until at last you hear that productive purring from the innards of the machine as the mechanism finally begins to run, announcing that the unknown is about to materialize. (1) (italics mine)

Due to the diachronous nature of this communication, there is always the threat of getting disconnected from one another, and once when Titus cannot send a fax message, he strikes the machine and it goes "dead." The medium is treated by the writer as if it is a live organism and its body and mind are inseparable: "While I was scribbling down my comment the fax machine-which was now working and collaborating tirelessly-rewarded me with yet another quick fax dangling head downward waiting to be read" (95).

On the other hand, Titus is sending his messages from Germany and he writes them in German first, and it is the machine which translates those message into Portuguese for Professor J, and here the machine leaves its mark by creating some awkward phrases like "think you not?" and "Oh God!"

The machine is also referred to as a "mechanism," which is the same term that Kirschenbaum uses for how the materiality of computers affects their functions in his book Mechanisms: "But after fifteen minutes of anxious attempts the mechanism accepted my note and my words flew (by what paths?) into Titus' hands" (Wilheim, 89).

\section{Physical Organization and Design}

The physical organization and design of Fax Messages from a Near Future is not that much different from the conventional print novels. All the words are printed in black ink on white paper, but there are two big differences in its physical organization which foreground the role of the medium and highlight its role in this 
novel. The first is the way the graphic surface of the novel is foregrounded. In this novel, Titus's messages have been distinguished from Professor J's thoughts and replies by the vertical line in the margin of the page and a different font. Here the print medium, which has its very own conventions for showing direct and indirect speech, is adapting another medium's features and in doing that, highlights the role of the medium and their different materialities in this process. Moreover, the whole process here has been facilitated by the use of computers in the book design.

Apart from this unique page design (by S\&W Design) the other way the graphic surface of this novel is highlighted is that there are no chapters or numbers to distinguish one part of the novel from the other. Instead, the writer has used time markers to separate one chapter from the other. The first chapter starts at 9:00 and the last one ends at 4:15 the next day. The reason for this division is the time that faxes are received through the fax machine. Such an arrangement does not necessarily succumb to the limitations that the book format imposes on the writing, and consequently the novel is not finished in the traditional sense of the word (the last fax of Titus is left unfinished in the middle of one of his questions).

On the other hand, from the very beginning, the fax machine leaves it mark in the way it physically (re)produces the document too. At the beginning of the novel Professor $\mathrm{J}$ wants to find out who sent him that strange fax and his only clue is the mark that machine leaves on the fax page, known as the "automatic record": "I glanced at the automatic record at the top of the fax in order at least to identify where it had come from: 427GMW411222" (5)

This number is repeated on the front and back cover of the book too, and though it seems to be a trivial detail in the novel, obviously it is important enough for the cover designer, Dan Mercer, to use it three times in his design in order to highlight the trace (and role) of the medium in the novel. The cover of the book shows a white page on which the title is printed in various fonts and has two big blotches of ink. On the top left and also on the back cover there is a "transmission error" message, which is another trace of the machine to show its failure to send the fax messages 


\section{Narrative Strategy}

The epistolary novel is one of the oldest forms of the novel and since a written letter is a record of the thoughts and ideas of the writer and both involve the writing process and the language medium, the writing medium is more evident in those works. Due to this similarity, epistolary novels have developed together with the writing technologies and, apart from the fax, emails and later texting technology from cell phones have found their way into novels which were written at the times that these technologies were still innovative experimentations. Similar to other epistolary novels, this one is also narrated through the correspondences of the two main characters but the big difference is that they are living 30 years apart. One of them is doing a research about the past and the other one is eager to know what is going to happen in the future.

The story itself is treated as a work of fiction by the writer. He discusses this issue twice in the beginning of the book: "We can surf on this ocean of uncertainty and feel the breeze of times to come. Relax. Let me tell you a story ... uncertain how to convey what had happened to me, I discussed a number of the topics with friends before taking the decision to hand over the following narrative to a publisher." (XI)There are no other metafictional features in this work and though there are various warnings in the middle of the story that transmission of the faxes from the future to Professor $\mathbf{J}$ may stop, the reader can easily browse the book to find out whether there will be more faxes in the book or not and where does the narrative actually end. The unique form of the pages which the fax messages have been printed on according to the time of receiving them, makes this task even easier.

\section{Reading Process}

The reading process of this novel is its most conventional feature; it is read like any other novel from the beginning to the end and the only thing that a reader has to do while reading this work is to turn the pages. Different fonts and the vertical bar at the left side of the page help the reader to understand whose fax they are reading and how they should differentiate between Titus and Professor J's faxes. The chronological arrangement of the narrative discourse makes the experience of reading 
linear and there are no other reading paths and the reader is not presented with any other options for reading this novel. 


\section{Conclusion}

Hypertext fiction joined the literary scene in the early 1990s and its early forefathers remediated print as closely as they could. The ability of the computer to link texts in a different media as well as the specific features of software like Storyspace enabled them to create works which were similar to works of metatextual print fiction and brought their materiality to the foreground. These works had more in common with books than the works which succeeded them. Links could be interpreted as the digital counterparts of footnotes, and their standalone feature meant that they were discrete objects like books This modality of the novel was short-lived, and a new generation of digital novels became possible because of rapid developments in computer technology permitting the inclusion of a variety of other media/modes in hypertext fiction, and the networked structure of the World Wide Web, brought about a new generation of digital novels. And, at the same time, print novels were also responding to these changes in information and communications technologies, incorporating them in their narratives and representing their materiality in changes in the form of print narrative. 


\section{Chapter Three}

\section{From Graphic Surface to Graphical User Interface, Metatextuality in}

Works of Web-fiction

... with the emergence of the World Wide Web, something new is happening. For those who've only recently lost their footing and fallen into the flood of hypertext, literary or otherwise, it may be dismaying to learn that they are arriving after the golden age is already over, but that's in the nature of golden ages: not even there until so seen by succeeding generations. (Coover, "Literary Hypertext") 


\section{Introduction}

By mid 1990s, hypertext fiction had become well introduced into academic circles through articles and seminars, but at the same time a new era in the age of electronic writing was beginning which had been anticipated by writer/scholars such as Joyce (1995) and had been thought of as a major threat which would sweep away the golden age of writing in the digital media and bring a silver age instead (Coover, 1999). These new changes could be divided into two related categories. The first category was the developments in the Information and Communication Technology (ICT) which enabled computers to host larger files and therefore made it possible to add a variety of other media such as video and recorded voice to the works of digital fiction whereas in the previous phase "audio and animation files were virtually nonexistent" (Coover n.pag). The limitations of the previous media for a writer/scholar like Coover had this advantage that made the "early hypertext...mostly discrete objects like book, moved onto low density floppies" (ibid.). As Coover states in the first part of his paper, in addition to the development of ICTs, something else which is called the Internet paved the way for the arrival of the works of web-fiction.

The main game changer in this phase arrived in the form of the Internet which through its various features such as the World Wide Web provided unique opportunities for writing novels within its networked structure. But what exactly were those changes and how did this new invention affect novel writing in the new medium? In order to answer this question, the main features of the Internet phase of the development of digital ICTs need to be discussed first.

The history of the Internet and how it developed out of ARPANET is not related to this topic and will not be reviewed here. What will be explored here instead is how the novel changed when it was transferred from the stand-alone programs which had been used to compose works of hypertext (like Storyspace) to the World Wide $\mathrm{Web}^{28}$. The Internet was commercialized in 1995 and from that year on provided different services like e-mail, instant messaging, Voice over Internet Protocol (VoIP) "phone calls", two-way interactive video calls, and the Web. Because

${ }^{28}$ The World Wide Web will be referred to as "the Web" in the rest of this chapter. 
of these features, the multimedia pages could now host a variety of modes in addition to written language.

The networked capability of the internet-which among other thing by separating the storage and performance could now be seen as a composition tool as well - and the multimedia features of the electronic medium made it easier for writers who wanted to use multimodal features (such as movies and music) in the graphic surface - or to use a computer terminology the graphical user interface - of their novels. What this meant for novelistic fiction was that its linguistic components were challenged by other features which could play a significant role in the narrative, and even the process of reading was challenged by the machine interferences. As we will see in this chapter, these two aspects were the major trends in the works of web-fiction. Digital fiction as the two volumes of Electronic Literature Organization show has expanded in various ways and includes a variety of works in digital media. The works analyzed in the case study section of this thesis provide a useful insight into the way these trends have been developed and demonstrate how the materiality of each work plays an important role in its narrative.

\section{Forensic, Formal and Networked Materiality}

The changes in the materiality of web-fiction can be analyzed in three major areas, forensic, formal and network materiality. Forensic materiality of the computers was enhanced by the developments in the computer technologies in the late 1990s and early 2000s. What these developments meant for the computers was that the other media/modes such as recorded voice, animation, and movie which were difficult to make and subsequently rare in the older systems became natural parts of the newer computers. As a result, computers became multimedia and this term became more dominant at this stage to refer to these machines in order to distinguish them from their antecedents. Several companies in their efforts to make computers faster, cheaper and capable of storing more data updated their previous models with innovative technologies to meet the requirements of those multimedia features. These innovations were accompanied by the so called CD and DVD revolution which replaced the older mobile storage media such as floppy disks, that in the previous phase were the popular form for data storage and exchange. The pace of these 
hardware developments was so quick that within 10 years, the novels published on floppy disks had to be replaced by a CD version (c.f. afternoon 2001, and afternoon 1990).

At the same time there was a big change in the formal materiality of computers which moved hand in hand with the changes in forensic materiality and its developments. The multimedia aspect of this phase meant that the new software developed at this phase were theoretically capable of bringing all these media together in a user-friendly format which allowed the user to move back and forth between these media. This requirement emphasized the role of interface and design in the software which will be explored in more details later in this chapter. Before discussing any of these issues any further, it should be said that there are two inter-related concepts within computing terminology that need to be explained, these concepts are textual user interface (TUI), and graphical user interface (GUI).

The computers in the previous phase of digital technology used purely script-based user interfaces for the machine-user interactions. This feature made navigation within the TUIs similar to navigating a book. The same way that reader finds the table of contents and then browses the book to find the specific place/page that she is looking for, the user of those computers had to choose one of the options presented to her linguistically so that the computer takes her where she wants to go. This similarity made the TUIs a naturalized part of the computers and since they were the only navigation option, no specific terminology was coined for them. The map view of Storyspace, as discussed in the previous chapter, was an important move from TUI into alternative interfaces which were not completely script-based. In this respect, map view was a step forward into the distinction between TUI and its successor GUI. On the other hand, the map view only was a navigation tool designed to facilitate the user's engagement with the text, and could easily be disabled (as it is in the afternoon). In this sense it was a peripheral feature and the user had to rely on TUI for reading the work of hypertext. The term Textual User Interface is a retronym that was coined sometime after the invention of graphical user interfaces in order to distinguish GUI from TUI. The term graphical user interface on the other hand highlights the importance of the design and how it affects what is presented by the graphical interface. Although in recent years, with the advent of the touchscreens the 
trend to create transparent user interface, or to use its computer jargon, Natural User Interface (NUI), has found more momentum, the GUI remains an important feature in the design of the works discussed, and in this respect becomes similar to the concept of graphic surface which has been discussed in this thesis. Analyzing it from a design perspective, Johanna Drucker (2011) defines interface as

a dynamic space, a zone in which reading takes place. We do not look rather through it (in spite of the overwhelming force of the 'windows' metaphor) or past it...The surface of the screen is not merely a portal for access to something that lies beyond or behind this display. Intellectual content and activities do not exist independent of these embodied representations. Interface, like any other component of computational systems, is an artifact of complex processes and protocols, a zone in which our behavior and actions take place. Interface is what we read and how we read combined through engagement. (9)

In this regard GUI in addition to serving its design role (or a physical resource) becomes a signifying strategy because, as Drucker aptly observes, the intellectual content and information do not exist independent of the physical resources in which they are instantiated. Looking at the GUI from this perspective makes us see its kinship with the graphic surface and how the web-fiction novelists by highlighting its role and presence create metatextual novels which expose their body and disrupt the reading conventions.

Another advantage of Drucker's definition is that it argues for the visibility of the GUI and by observing "interface is a dynamic space, a zone in which reading takes place [which we] do not look rather through it or past it" she claims that GUI should be treated as a surface without depth. This claim might seem confusing at many stages. Despite the pages of the book, the computer has a depth which exists beyond its screen, but what lies beyond the GUI is not what the human user is trying to reach, and various layers of code which exist at that space are meant to be read and executed by the machines, not the human user. What is available for the user is the interface. As discussed in the previous chapters, what lies beyond the surface of a 
print page is empty space, and though here the empty space is replaced by the space occupied by hardware, it can be said that nothing apprehensible for a user without the technical knowhow - which is the majority of users - exists beyond the GUI. So the GUI becomes a surface where "the reading takes place." This understanding of GUI highlights its role in the design of the works presented through the electronic medium and as it was mentioned above, the works of web-fiction by using graphic devices in their graphic surface continue the trend of metatextual print novels.

\section{Materiality of the Internet: Computers and Network,}

The blindness toward the materiality of the electronic media, as discussed in the previous chapter, showed there are still "gaps in our understanding" about the digital media. Whereas the materiality of the computer could be seen and explored by analyzing its forensic and formal materiality, the materiality of the Internet might not be as evident as the materiality of the computers. The materiality of the Internet is explored by Paul E. Cerruzi in his 2006 essay with the same name. In that essay, Cerruzi argues that the invisible structure of the Internet has led to "gaps in our understanding" of its materiality. He argues that the materiality of internet resides in "physically small objects: silicon chips the size of a fingernail that require a microscope to view their circuits, and fiber-optic cables thinner than a human hair that, even with cladding, are little thicker than ordinary coax cable" (96). On a closer look, what Cerruzi is hinting at is the same forensic materiality that Kirschenbaum has discussed in detail in his book. In this sense the materiality of the Internet is that of the interconnectedness of computers whose materiality was explored in the previous chapter. What distinguishes the materiality of the Internet from the materiality of a single computer is its networked structure, which among other things allows a differentiation between the storage and performance. This feature, as Hayles has also pointed out, makes it possible to store files on a computer, and retrieve them from another computer "across the globe" so that a digital text which includes words, images and movies, can perform what it is designed to do by bringing together those files from the servers, which are the computers that are used for storage of the files. Ceruzzi observes that the distributed hubs of these servers across the globe, can have serious implications for artists. Though Cerruzi might not have been aware of the first volume of the works of ELO which has been published in the same year, reading 
those works makes it clear that Cerruzi did not need to wait long before seeing those implications that he had predicted in that essay. As we will see, the majority of the works of web-fiction do not have one central character or one main narrative. The novel in this environment becomes a network that each reader can explore by starting at various points and then moving further according to her own selections and the navigational methods offered by the novel through a variety of media/modes.

An important feature in the materiality of the Internet, which is absent from Cerruzi's essay, is the machine-to-machine interactions which are initiated by the human user. These interactions usually come to the surface when they fail to do what they have been supposed to do. For instance when the user clicks on a dead link, that is a link whose server has been changed, or a link which refers to an obsolete webpage, one of the annoying 400 or 500 series of Hypetext Transfer Protocol (HTTP) messages might appear on the screen. The evidences of machine-to-machine communication have been narrowed down to codes which make it possible for the communication to happen between computers, and these codes are the building stones of the language for the communication between networked computers. Although several languages have been created for this communication, the lingua franca for the computer turned out to be HyperText Markup Language, which as the previous quotation from Ensslin shows became its "major programming language." So the next step in understanding the materiality of the network is having a closer look at what HTML is and what its major characteristics are.

\section{Code and New Languages of the Computer}

There are several definitions for HTML, but for the purposes of this thesis, one of the most useful ones is presented by the editors of Electronic Literature Organization (ELO). In their definition HTML is

... a format for defining web pages to be displayed in a web browser. HTML is used to structure information-to designate headings, paragraphs, and lists, for instance, as well as hypertextual links-and can be used to describe, to some degree, the appearance and semantics of a document. Dynamic HTML or DHTML is used to refer to interactive web sites that use other technologies in addition to HTML, 
such as a client-side scripting language (usually JavaScript), and that manipulate pages using Document Object Model and Cascading Style Sheets (CSS). (ELO Website)

By defining HTML as a "format" this website highlights an important feature of HTML, which is its role in structuring the information. In this sense, although the user does not see the codes of HTML, these codes are responsible for the way data is arranged on the page and how a webpage is to be "displayed" or formatted in a web browser. This important role of HTML in the materiality of the webpages, has encouraged some web-fiction writers to experiment with the machine language and particularly HTML. These writers combine the language of human interaction, either English or any other language, with the machine language by bringing the codes from the infrastructure which it created to the foreground. Some of these works include Bad Machine (2006), Code Movie 1 (2006), Internet Text (2006).

What made HTML the popular language on the web was the way it provided access to create multimedia pages. As Ensslin states:

The key achievement of HTML as a standardized mark-up language was its propensity to create a variety of different semiotic systems, previously analogue, i.e. separated in terms of mediality and materiality, on the basis of unified, script-based programming code. These semiotic systems comprise text, graphics, digitized speech, audio files, pictographic and photographic images, animation and film. $(21)$

This capability of HTML made it possible for writers to use several modalities in their works of web-fiction. This feature has been used so intensively that it has become one of the major trends and distinguishing features of the works of web-fiction. When there is an abundance of material available for representation, the way these materials are combined and their combinations become important. In the same sense that a novel was a combination of already chosen words presented in the codex format, and hypertext fiction was the result of combination of different lexia (each a result of already chosen words to be placed alongside each other), here the 
combination occurs at the level media/modes, and the writer can choose to present one part in movie, another in words and a third one through music.

\section{Electronic Text and Dynamic Layering}

The concept of layers might seem a paradox in this discussion, but this geological metaphor is quite useful for describing the way works of web-fiction function, and it is a widespread term among computer scientists. This term allows us to consider how the materiality of works of web-fiction plays an important part in their performance which is to present their narrative. Hayles uses the term layered for digital texts in the sense that they include a multitude of invisible activities that occur at the level of machine

... generating the text that appears on screen are cascading processes of interpretation, from the hypertext mark-up language tags that format text on the World Wide Web, to compiled/interpreted programming languages like $\mathrm{C}++$, down to the mnemonics of assembler, and finally to binary code and the alternating voltages with which it is associated. (Hayles, Electronic Literature 163)

Hayles' definition is useful for those who want to go deeper into the infrastructure of the machine, because as can be seen from her explanation, she goes further into the workings of the machine in her analysis, whereas this thesis focuses on the visible aspects of the digital texts. In the previous sections, it was shown that by using HTML codes designers format the interface of the works of web-fiction so that the different media may be placed in the same space. The network and the servers made it possible to retrieve files which have been stored on faraway computers to perform in the same interface that the other media are. However, there is no easy relationship for the existence of these media/modes in one specific place and they have different kinds of relationships to each other that the metaphor of layering helps to clarify. If we think of every media/mode as a layer, different layers of media/mode have been placed on top of each other here. The dynamic relationship of these layers means that there is no pre-determined top-bottom relationship among them and at times all of them can coexist on the surface. 
When the reader of a work of web-fiction opens up the work in her browser, HTML assembles the interface by reading and executing the codes designed for this activity, and in this way creates the interface by bringing up the layers which have been designed to be placed on the front page. What happens afterward through the user interaction with the machine depends on whether the user orders the reading or the machine orders it for her. These two features lead to two different trends in the works of web-fiction which will be analyzed in more depth in next section of this chapter. What needs to be emphasized here, in respect of the layers, is that these layers of media can have a variety of relations to each other, but because of their presence alongside each other in a work web-fiction they find new meanings and interpretations that are determined by their place in the network of relations and this is what Gass, Patrick Joyce and Bennett have emphasized in their discussion about materiality.

Another important aspect of HTML is that it enables some other multimedia and cross-platforms such as Shockwave or Flash to be used to create works which would have required lengthy codes to be executed in their current format if they had been written in HTML codes. Thirty two out of the sixty works in the first volume of ELO and twenty nine out of the sixty three works of the second volume have been written with either Shockwave or Flash. Other authoring programs or languages such as Inform, Java, Javascript, Quicktime, Squeak, etc. share a smaller share in the ELO collection of the works. Although the popularity of Flash does not equal the popularity of Storyspace for the works of hypertext fiction, the reason for this popularity remains almost the same. Similar to Storyspace, Flash makes it easier to for writers who are not programmers to create works of digital fiction.

\section{Web-fiction: Cybertext and Multimedia}

Now that the materiality of the works of web-fiction has been explored, it is possible to propose a definition for these works. Although being presented on the Web, is one of the qualifications for a work to become web-fiction, it is not the only one. In the previous chapter, it was discussed several critics had suggested definitions for the works of hypertext and web-fiction. In the latter stages of writing this thesis in 2012, one particular definition was suggested by a group of critic (Bell et al) which 
fits quite well with the issues raised about digital web-fiction. According to these critics web-fiction is "fiction written for and read on a computer screen that pursues its verbal, discursive and/or conceptual complexity through the digital medium and would lose something of its aesthetic and semiotic function if it were removed from that medium" (Bray, Gibbons, and McHale 471). This definition includes both the multimodality aspect (verbal, discursive and/or conceptual complexity), and the cybertext aspect (non-transferability) of the works of web-fiction. Each of these aspects will be analyzed in more details in the rest of this chapter.

As discussed above, the material features which assemble the text, work together to create the interface of the novel which is first point of (virtual) contact between the reader and computer. Any work of web-fiction has a unique interface from which the reader can start her path in the novel. What happens immediately after a reader engages with the text is that either the user finds herself subject to the functions of the machine and realizes that the machine directly interferes in her reading or she retrieves the various layers of multimedia in order to construct/follow a narrative. Each of these trends poses different questions for the narrative and the novel. The first trend keeps its kinship with the metatextual novel because it disrupts the reading conventions and places it at the mercy of the machine functions. The other trend examines the presence of other media in a genre which has been dominantly linguistic, and by doing so preserves its kinship with metatextual novels. In other words these two trends are divided into those which lay emphasis on the act of reading and those which stress the presentation of the story. The first group stresses the performance of the narrative in the Web and the second aims at presenting the narrative through the multimedia features of the Web. For the ease of reference, the first group will be named cybertext novels and the second group will be named multimedia works of web-fictions.

\section{a. Cybertext}

The closest term for those novels which disrupt the reading convention and make the machine an important part of their reading process is Espen Aarseth's "cybertext" which is the term that Aarseth's uses for "texts that involve calculation in their production of scriptons" (Cybertext 75). This term has been also used by Ensslin for almost the same concept that has been discussed in this thesis. According to 
Aarseth, scriptons are "strings as they appear to readers" (ibid. 62) and in this sense they become closer to the way the layers have been used here. As Aarseth observes this term can be used in its broad sense for works like afternoon too but the way it is used in this thesis slightly differs from such usage. Here the term is used to differentiate the works of web-fiction which stress their materiality through the performance of their text from multimodal works of web-fiction.

Cybertexts make the machine an integral part of their materiality and the machine functions directly interfere in their process of reading. Ensslin has a similar definition when she categorizes the works of electronic media into three generations of hypertext, hypermedia, and cybertexts. She defines cybertexts as "hypertexts that are programmed in particular ways as an autonomous 'text machines' that assume power over the reader by literally 'writing themselves' rather than presenting themselves as an existing textual product" (22). Ensslin's definition implies autonomy from the author, because what the reader encounters is merely the text written by the author. An authored text has always assumed power over the reader, but now machine writing has brought about a different conception of the text/writer as including a machine element which because of its programming can produce text effects not already fully written into the text. In this sense, Ensslin's definition becomes similar to the way cybertexts has been defined here because for Ensslin too, the reader has to compete with the machine for the control over the text which appears and disappears and it is through this engagement and competition that the reading process moves forward.

There have been a variety of ways by which the cybertexts have demonstrated how their materiality emerges through the interaction of the user and the machine during the past two decades. A short survey of the works of the both volumes of ELO and other works of web-fiction on the World Wide Web shows that one of the most-used techniques to achieve this end has been placing limits on the time available for reading any lexia before it disappears from the screen. Hegirascope (1997) and Chemical Landscapes Digital Tales (2006), are works that introduce time limits to the act of reading; in these works, new webpages replace the previous ones after a specific amount of time which is usually shorter than the time required to read the whole page. 


\section{b. Multimodal Web-fiction}

Multimodal works of web-fiction, which make up the majority of the works in both volumes of ELO collection, seriously challenge the dominance of the written language as the only mode for novelistic fiction, and in this respect they can be aligned with the tradition of metatextual novels in print which flaunted their body by emphasizing their graphic surface that made non-linguistic elements such as photos and colors, part of the text. These works are composed of different dynamic layers of modes which have different relations to each other and co-exist at the level of the interface. An important implication of this multimodality is that the structure of these novels can be interpreted as reflecting the structure of the Internet. As Ceruzzi had argued, there is no central computer that controls the whole internet, similarly the multimodal novels in ELO collection do not have a central character and there are multiple discourses and multiple stories which eventually create multiple readings. 10:01 which is analyzed here shows this distributed architecture of Internet and its mosaic structure by having what in theatrical and cinematic practice is known as "ensemble cast." Another work which makes this distributed structure and dynamic layering more literal is Dreamaphage (2006) by Jason Nelson which presents itself in $3 \mathrm{D}$ and the reader has to drag up or down the layers of 3D text in order to move forwards and backwards within different levels. Reconstructing Mayakovsky (2011) by Illya Szilak in its 3D format includes a multitude of interpenetrating dynamic layers which is described by the ELO website as "invitations to imagery theater events, warped Google searches, a manifesto addressed to the future of humanity, a propagandistic movie in support of 'Monad Technologies' and other less-definable digital genres." Moreover there are several links to the webpage on the Internet which take the reader to webpages specifically designed for this novel on the Web and in doing that, emphasize its networked structure which stands in sharp contrast to the "discrete objects like book" status that Coover had praised while taking about works of hypertext fiction. This opposition to making a novel a discrete object, as we will see in the next chapter, is later picked up by metatextual print novelists like Danielewski to create networked and multimodal print novels like House of Leaves. 


\section{Conclusion}

What happens at the level of the interface in the works of web-fiction is a direct result of the human interaction with the machine and the machines interacting with each other so that the text is generated in this process. Cybertexts directly interfere with the text which is presented to the reader, whereas the multimodal works by allowing the reader to move freely between their layers of multimedia highlight other issues, for instance how the linguistic aspect of the novel engages with the other multimodal aspects of the novel.

Each of the case studies analyzed in the rest of this chapter shows an example of the issues discussed above. They are both written by Flash and use a variety of features of the Web and HTML. Chemical Landscapes Digital Tales is a cybertext which restricts the time allowed for reading its webpages to 10 seconds only. 10:01 on the other hand, in its digital form uses a variety of media/modes which appear alongside each other. Although linguistic parts of the text carry the main narrative load, the spatial arrangement of its interface creates an interrelated arrangement between various modes. Since this novel has had two instantiations, one in print and one on the Web, the print version of this novel has been analyzed here as well to highlight the differences of each instantiation. The print version of 10:01 highlights its graphic surface too, but the temporal arrangement of the mini-narratives makes it possible to have more mini-narratives within the novel to make up for the non-existence of the other modes in the novel. 
Case Study 3.1. Chemical Landscapes Digital Tales (2006)

\section{Introduction}

Edward Falco's Chemical Landscapes Digital Tales is a cybertext and as its name suggests has two parts, the chemical landscapes which according to Falco's explanation at its webpage at ELO website, is a "series of photograms by Mary Pinto. The photograms suggest landscapes but are created entirely in the dark room, using only chemicals and a flashlight." The "digital tales" is the second part of this work which is what Falco has written based on what is "suggested by the particular chemical landscape." These two are brought together in a website designed by Will Stauffer-Norris.

These landscapes in a process which resembles photography but differs from it have been generated chemically, and stress their non-representational status. In this process light has been emitted from a flash light to the chemicals in a dark room. The digital tales refer to the stories which Edward Falco has written for these photograms. These tales as the meaning of the word tale implies are short narrative works which appear and disappear on the screen along with the eight other photograms. What distinguishes these tales from the other tales is that these machine-generated tales are made to disappear before the reader has enough time to read them. By assigning the name of landscape to those swashes of color, the writers are giving those works a value which is arbitrary, just like the conventional relationship between material form of the signs and the concepts to which they refer. While there is no obvious relationship between these two, their presence together in this works highlights that what has been established as the meaning or reference of a sign is maintained by tradition. The author encourages this association by stating: "I hope the relationship of language and narrative to the 'tale' parallels the relationship of light and chemicals to the 'landscape." In other words, the author here is trying to "parallel" the way the images have been created by fashioning narrative in way that shows that neither image nor words are directly referential, and the meaning which is assigned to them exists by convention. This puts them in complete contrast to traditional novels in which the assumption is complete authorial control over the text. In this work authors do not claim complete authorial control, and instead underline the role of chance 
(there is no order/sequence to determine where/which part of the screen the reader should click first) and the control exerted by machine over the act of reading.

\section{Physical Organization and Design}

An important aspect of Chemical Landscapes Digital Tales is how it separately acknowledges its three main features (the linguistic section, photograms, and the digital materiality, that is its website design and other machine functions and dimensions) by bringing the different names which have been responsible in creating each of them on its graphical user interface. On the level of formal materiality this work takes advantage of the HTML and Dynamic HTML (DHTML) in order to present its GUI through the Flash platform. The introduction page of this novel, which has now become the standard design for all the works published through ELO, contains the name of the work, its writers, a short description about the work in a white margin right below the name of the work and its authors, followed by a lengthier "author description" section. On the left to this section is a small shot from one of the scenes of the novel and to the right of this section is the BEGIN button. Apart from the BEGIN button, the rest of these features can be found in print novels too, but what is unique about this novel and the other works of web-fiction is the four sections which follow this part. The first one is the "instruction" section which guides the reader how to read this novel. This section highlights the different materiality of each work and what is the expected reading process and what should a reader do to be able to initiate the dynamic relationship with the physical resources of the work so that the materiality of the work emerges through this process and presents itself to the reader. Since some software such as Adobe Flash and Shockwave are updated regularly through the website of their manufacturing companies, the guidelines at this section notify the reader which version of which program is the bare minimum requirement for reading this or that work.

Since ELO volumes are collections of digital works of literature, some of these works might have already been published on the website of their writers or designers. Thus the heading for the next section is "previous publication," which does not apply to this work because it is the first time it is being published on the Web. The copyrights for these works through Creative Commons License have been placed 
below this section. At the bottom right of the lexia and right above the seal of Electronic Literature Organization, there are two series of words, the top one the key words for this work which include "Collaboration, Fiction, Flash, HTML/DHTML, Time-Based, Visual Poetry or Narrative, Women Authors." These keywords are links which make it easier for the reader to find more about each of those words and what they signify. Also it is possible to find the works which have a common feature, such as Flash, for instance, under the definitions of these works. Moreover, these keywords can help the reader to have a quick glance at each of those works and find out what each of them are about.

All these keywords here, except the last one refer to the work itself, and the last keyword refers to Mary Pinto who is one of the trio involved in creating this work. The bottom line which runs parallel to the keywords above, refers to the website, and the collection and through the five keywords in that section-All Keywords, All Authors, All Titles, About, and Main Index-the readers can search/explore the whole collection and read about the editors, designers, sponsors etc.

After the introduction page, comes the front page with one of the photograms as the background. The names of the people involved in creating this work and website have been written on the top left of the sky part of the photogram. Almost all of these photograms are divided into a sky section at the top and another part (presumably land) at the bottom section. It is not possible to click on the sky section, but the land section is divided into eight parts, and depending on where you click, you are taken into one of those photograms. Clicking from left to right, the photograms appear as follows:

Top left: "Through this field..."

Bottom left: "Rain"

Top middle left: The interior of dreamer's eye

Bottom middle left: Placid

Top middle right: Rough Seas 
Bottom middle right: Sky overhead

Top right: A blue spine of land

Bottom right: I

There is no particular sequence in the way these photograms are linked to each other and the order mentioned above is not made obvious by the text. After several readings, the place of each photogram on the screen can be figured out as it is mentioned above. However, this does not make any difference to the notion of chance as distinct from authored sequence because following the order stated above does not produce a narrative sequence with some sense of completion or closure.

\section{Chemical Landscapes Digital Tales}

Chemical Landscapes (photograms) by Mary Pinto

Digital Tales (fictions) by Edward Falco

Site Design by Will Stauffer-Norris

Figure 3.1. A screen grab of the GUI for Chemical Landscapes Digital Tales

The design of this work brings together these eight photograms with their ensuing "tale" under the GUI which itself is number four on the list. Such a design 
incorporates the GUI into the work by making it both the navigation tool and a part of this navigation. By doing that, it makes GUI a "dynamic space" and in this sense it becomes, in Drucker's words "what we read and how we read combined through engagement."

Since the organization and the borders of each photogram do not follow any specific orders, it is possible to access any of the photograms without going through the others first. The dynamic layering in this novel works in a more literal way because it brings a layer of image and a layer of words together, keeps them together for a short while, and then takes them apart again.

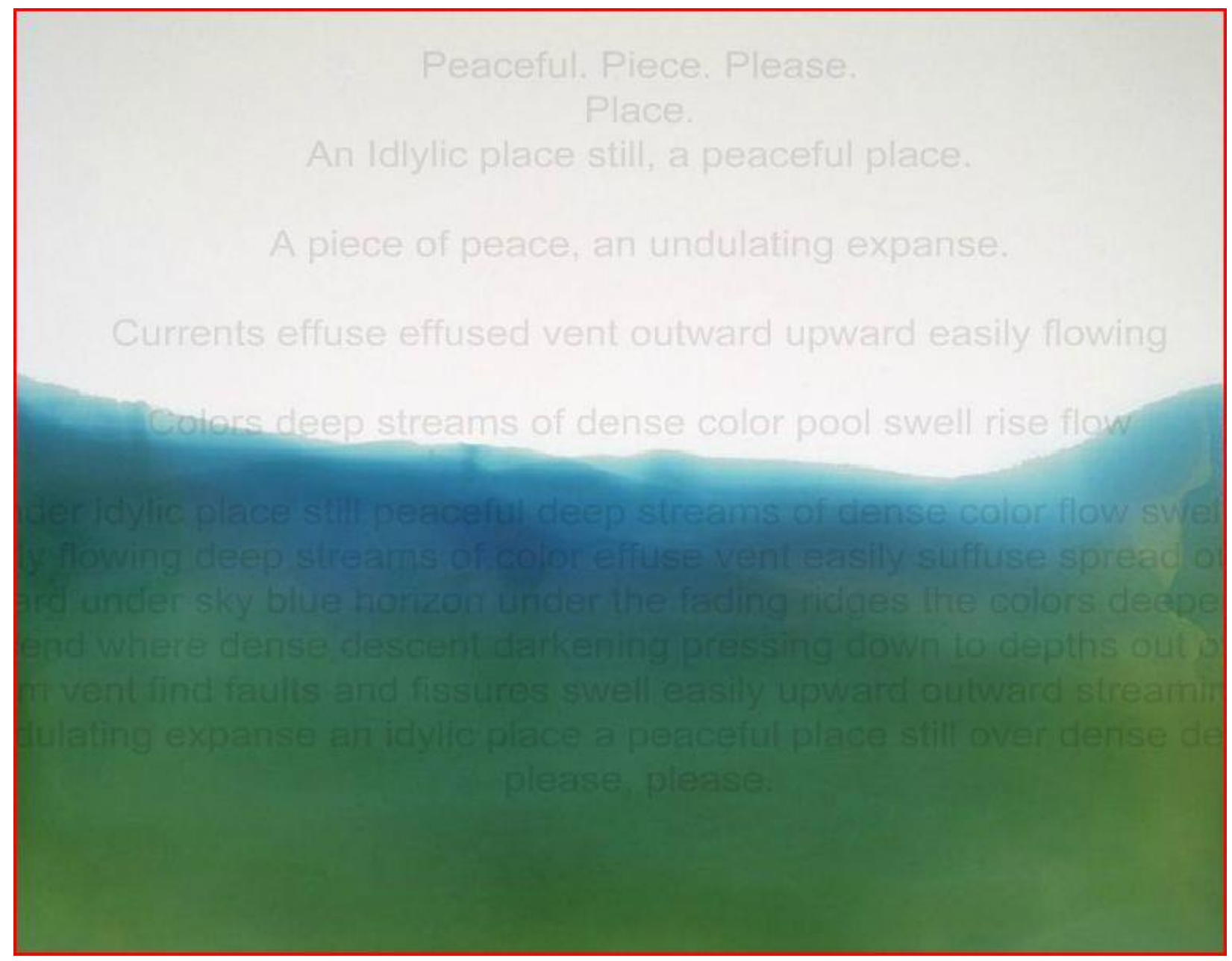




\section{Placid. Idylic. Still.}

Peaceful. Piece. Please.

Place.

An Idlylic place still, a peaceful place.

A piece of peace, an undulating expanse.

Currents effuse effused vent outward upward easily flowing

Colors deep streams of dense color pool swell rise flow

Under idylic place still peaceful deep streams of dense color flow swell rise easily flowing deep streams of color effuse vent easily suffuse spread outward upward under sky blue horizon under the fading ridges the colors deepend and descend where dense descent darkening pressing down to depths out of which stream vent find faults and fissures swell easily upward outward streaming to an undulating expanse an idylic place a peaceful place still over dense depths, please, please. 


\section{Placid. Idylic. Still. \\ Peaceful. Piece. Please. \\ Place. \\ An Idlylic place still, a peaceful place. \\ A piece of peace, an undulating expanse. \\ Currents effuse effused vent outward upward easily flowing}

Colors deep streams of dense color pool swell rise flow

Under idylic place still peaceful deep streams of dense color flow swell rise easily flowing deep streams of color effuse vent easily suffuse spread outward upward under sky blue horizon under the fading ridges the colors deepend and descend where dense descent darkening pressing down to depths out of which stream vent find faults and fissures swell easily upward outward streaming to an undulating expanse an idylic place a peaceful place still over dense depths. please, please.

Figures 3.2. - 3.5. Three stages of the appearance and disappearance of the text on the screen in Chemical Landscapes Digital Tales

An important feature of Chemical Landscapes is that it tries to distinguish its webpages from lexia. For Joyce, lexia was one stable part of the network which was part of the whole narrative of the novel and could be quite short or quite long. However, the lexia here is transformed into an unstable page which exists in absentia, it only appears when it is summoned by the reader and then disappears right before her eyes before she has had enough time to read it.

The time of the reading becomes quite an important feature here, and dictates a specific kind of reading from the reader. This puts the readers in a completely different experience of reading from what the early readers of afternoon had experienced. For them the text was stable and they wanted something more, so they 
kept clicking until they became bored and stopped reading. Here the reader must be completely attentive to the text to be able to read a little part of it.

What the writer aims to achieve by adding the "reading time" factor to his novel is to highlight the graphic surface of his novel and by doing that draws attention to the role of the medium that instantiates his work. By making the part of the text which traditionally provides most access to meaning unstable, or in other words, unavailable for as long as the reader needs or wants to attend to it, the instability here is transferred from the reader's length of attention to the text's length of appearance which opens up a consideration of the way in which all experience is fragmented, imprecise, and remembered in flashes. Therefore, that which we claim to know from it rests on imprecision and incompleteness.

\section{Narrative Strategy}

As observed in the introduction to this work, the linguistic components "like the images, are abstract, mentioning an event or scene and inviting readers to imagine the characters and relationships involved." In this way, abstract chemical landscapes parallel the status of language itself. In the same way that an abstract painting is open to various interpretations without any one of them being the final interpretation, the response of each reader to narratives suggested here by the scenes or events mentioned can be different and in this way the work becomes more similar to the works of minimalist painters and writers.

The abstract landscapes, and the words which are meant to parallel their abstraction, intensify the opportunities to construct narrative discourses and stories out of the words and phrases which are presented on the screen for a short time. The narrative here becomes the result of the choosing of words from different parts of the screen and their coming together which may or may not create a context for their collocation. This kind of arrangement is a direct result of the dynamic layering which now occurs at the level of the linguistic section. The group of words written for each photogram is retrieved on the screen through a single click, then the few words which a reader can read in that short span of time along with the accompanying photogram can suggest a narrative or a part of narrative. So the narrative has to be built piecemeal, and it is the reader who has to bring those words together and move them 
back and forth, or in other words arrange them according to the way she thinks would make more sense, so that a narrative is constructed.

Another aspect of this abstraction at the linguistic level of the novel is that it becomes more similar to flash fiction and minimalist works in which the reader has to fill in the gaps. In this sense the narrative becomes a possibility within a work that requires the reader to actively engage with both the medium and the linguistic signs within that medium to create it. As we will see later in The Fifty Year Sword too, the backstory and many other elements within the narrative might remain obscure, but the reader has to come back to the same page to read what is there more closely, but as Ciccoricco (2012) has argued, revisiting those pages brings the close reading of a text to the foreground.

Also the endless possibilities of narrative in each of these eight pages make it feasible to think of this work as "novel." As we had seen in Gass, and we will see in the next case study as well, as soon as the physical resources are brought to the foreground and their role in the signification process is emphasized, the narrative changes from the way it has been used in traditional novels, instead of focusing on one or two central characters, it focuses on a variety of characters. Although this is not something new and has been used in classic works such as 1001 Nights, it resurfaces again in the works of metatextual novels. The eight pages here have around 200 words each and though the overall number of words in this work might not satisfy those who categorize a work as a novel according to its word count, the possibilities of the narrative here expand in a wide variety of ways and this is how this novel justifies the claim for it being a novel. Here a few possible stories are presented.

Story (1)

The narrator (I) is in a deeply distressed state of mind following the ending of a relationship, but tries to keep outwardly an appearance of calm and engages with his feelings only in dreams.

Story (2)

The narrator (I) is alone following the ending of a relationship. He tries to find a correlative in nature for his feelings, but has to recognize that natural events have no 
actual, but only a perceived, connection to his state of mind. The place of their convergence is the border between land/sea and sky, which is also the border between objective and subjective states of affairs, which he maintains by appearing outwardly calm. It is only in dreaming that his subjective situation becomes accessible to him; his dreams manifest the extent of his sense of loss, as he can only recover anything of her in dreams, and those drams are mostly associated with anger, sickness, emptiness. The one exception is a dream of childhood, of a boy swimming and experiencing complete freedom. But he knows that dreaming cannot change his present situation, and that the hope implied by the change from storm to calm in nature does not transfer to human experiences.

It goes without saying that these stories are highly personalized constructions out of the possible pool of stories, and other postgrads and colleagues created other stories which range from the story of Genesis to that of a guy who is hallucinating on a stretcher. This wide range of stories becomes possible because each photogram is accompanied by a unique layer of words which becomes available on the GUI for a short time and then disappears. So this abstraction has resulted in a work which through engaging its materiality in the presentation of its narrative has opened up a wide variety of possible stories or in Gass's words "pool of stories."

\section{Reading Process}

The reading process of this novel is explained at the introduction that Falco, its author has written for it:

My hope is that the reader will recognize the necessity of jumping around in the text, picking up pieces of the tale to read and ignoring other pieces, thereby creating a different experience with each reading. If you think of reading a traditional story as a journey with a beginning, a middle, and an end, then reading a hypertext is like walking through a field: readers begin at any one of several different starting points, wander around as long as they like, and then exit wherever and whenever they choose. (ELO Website) 
The metaphor of walking through a field, as the writer mentions, means that there are no pre-designed paths to follow. Also, as we saw above, you do not necessarily need to visit certain pages in order to get the permission to visit other pages which the writer has placed behind some restrictive system such as guard-fields. You can run in any direction and visit anywhere that you want to. Here the work asks to be read through selecting the pieces, and openly claims that the narration of a work of web-fiction can only be served piecemeal, one bit from here, one bit from there, and you always know that your reading is incomplete and you have to create a narrative from those pieces which you have already read.

The time limit adds a temporal dimension to the text which shows that in the same way that narrative in a novel moves in time, the act of reading is also time-based. However since the allocated time for each screen is significantly shorter than the time required to read it, Chemical Landscapes Digital Tales works against normalizing the act of reading and providing the reader with the ease of reading a novel. In other words, the reader has to constantly engage with a medium which at the same time reveals the story and then makes it fade away before her very own eyes. Since the reader cannot click anywhere on the sky section of the work, and the story only appears by clicking on the land/landscape section of the screen, the writer is also implying that the stories do not exist in the immaterial realm of the sky (as opposed to the material realm of the land), and it is through engaging with the material medium and the body, that a (potential) story takes place; these stories may be about the skies or the land, but it is the land which can be the originator of the story, not the sky above. While the text is brought up, the background turns into white and in a way it parodies the act of reading a print work (black on white), and its ephemerality in the culture, as Joyce had said that book is a momentarily instantiation of the network.

As has been discussed in the first chapter, many writers of metatextual novels have attempted to show that the fictional represented world is not a real world and their artifact has been designed and made by the author. Gass emphasizes this point while referring to the character in a novel and the metaphors which have been discussed in the first chapter. In a similar way, the narrative strategy of this novel does the same thing. As soon as the reader clicks on the bottom part of the screen, one of the eight narratives emerges and then fades into white and fades away before it 
allows the reader the chance to fully read it. Therefore, the representation moves toward de-presentation and brings the role of the medium, which is the interference of the machine here, to the foreground.

The non-transparent status of the novel is also stressed in this work. By creating something through an artificial method, and then naming it a landscape, one is actually paralleling the act of creating a fictional world and giving its agent/characters and settings familiar names. It is an effort to create a world which is not the outside world, but has certain similarities to that world, in the same way that those landscapes are not real landscapes, but have similarities to the landscape as such.

\section{Conclusion}

Every novel at its best represents an abstract (fictional) landscape in the mind of the reader and this is something that the figural covers of most books betray. The represented fictional world which has been given shape by the author only takes shape in the mind of the reader through the reader's engagement with the text. The underlying physical organization of this work is a window toward the medium, and in that sense it becomes similar to Gass's metaphorical statue. The same opening frame is repeated in the reading to remind the readers of the experience of reading and how it is similar to the looking at a statue. The gaze of the reader moves along the statue, but after a short while, the front page re-inserts itself in the experience of reading. In fact, reading is different here in the way that it involves engaging with the medium rather than seeing through it.

This novel comments on and criticizes the way earlier writers of hypertext fiction had divided up their lexias for the readers, imposing a rhythm for reading which as Aarseth depicts is more restrictive than the rhythm imposed on reading by the print novel. As Joyce has already shown, dividing chunks of texts written by and for the computer is arbitrary, and there are no hard and fast rules for how many words should be there in a lexia (as opposed to a page of a novel which almost contains the same number of words as the other pages), and though some lexias are densely filled with words, others contain fewer words, and there are even some with only one or two letters in afternoon. The normal practice for a reader of a work like afternoon is that 
she should read to the end of the lexia and then click on the words which yield. However, Chemical Landscapes Digital Tale eliminates this spatial arrangement and exerts a temporal limitation on the act of reading which allows only a couple of words to be read before it fades out. This feature makes media more visible and makes it a part of the message it is aiming to convey. It also forces the reader to make a mental connection between the photogram on the screen, the words which he has read in his previous reading, and the words which he reads at that specific moment when the text fades in again. 
Case Study 3.2. 10:01

\section{Introduction}

10:01 by Lance Olsen (and Tim Guthrie in its digital version) is about random people sitting in a theatre in Mall of America watching the trailers ten minutes and one second before the feature film starts. The novel offers its own metatextual format and representation as a critique of a dehumanizing capitalist society which forces people to become consumerist machine in the service of a rich minority. The narrative takes advantage of a wide range of the capabilities of language and book itself in its print instantiation, as well as language and the digital medium in its electronic version which has picture, sounds and movies.

This novel was first published in print in 2005 and then Tim Guthrie "brought [it] into machine" in the November of the same year. A comparative study of this novel in print and electronic media will reveal how the instantiations of this work in these two different media differ from each other and how each one by highlighting its graphic surface follows the line of metatextual novels. The print and digital versions of this novel offer two different experiences of reading in each instantiation. In the "note" which is placed in the beginning of the print version, Olsen encourages such a comparison for the two versions of the novel. Immediately after the title page of the print version, there is a page entitled "Note" which has the address of the complementary hypermedia version of this on the website of cafezeitgeist.com. However upon going to the website: the reader will encounter the following question:

LITERATI: You talk of pushing reader instability/uncertainty farther in 10:01 than you had in Girl. Is this because you raise that third text of hovering between the paper and electronic iterations of 10:01, and do you think readers will automatically sense this between the two readings? (Cafézeitgeist.com) 
Although Olsen does not provide any further explanation of this "third text" what can be inferred from that quotation is that print and the electronic version are meant to be read alongside each other so that every reader finds that "third text of hovering between the paper and electronic iterations of 10:01" for herself. The main advantage of such a reading is that it establishes a common ground for seeing the different forms that 10:01 finds in each of instantiations (or iterations as Olsen puts it). It is through this comparison and contrast that a third text appears that can be closer to the electronic version or the print version for any reader, and this is why this third text does not have a specific place and while it shares characteristics of either text, it is not totally print or electronic, but hovers between each of them.

Café Zeitgeist which according to the short biography at the end of the book is the online resort of the author is a website designed to challenge the capitalist epistemologies which are called The Great Illusion. A full discussion of the Great Illusion is not within the scope of this thesis, but its relevance here is that the author in this novel is trying to present alternative ways of story-telling rather than following the already established and accepted modes which favor a hierarchical structure in their presentation. In the same way critiques of capitalism offer alternatives which look to replace it with other systems that are not inherently dependent on the hierarchies, and in which human beings are not just robots who have to fill in the time slots or consumers who sit in a cinema passively watching someone else's movie.

The main strategy which the novel employs is eliminating the hierarchical structure that divides the characters in a work of fiction to major and minor characters. Such a structure exists in almost all works of traditional fiction. The hierarchical relationship in traditional novels reinforces the notion that hierarchy is inherent and inevitable in society and those that are at the bottom of this hierarchy are not as important as those who are at the top, and they merely exist there to serve the main character(s). However, in this novel the author has used a random selection of people and thereby created a social environment where anyone who can pay the admission fee can enter into it. Therefore, in 10:01 all the characters are equally important and there is no hierarchy between them. 
On the other hand, the dominance of the narrative language, a specific sub-category of written language that mainly uses the past tense and is narrated by the first or third person narrator has been undermined in 10:01. This novel uses other forms of language such as present tense, numbered sentences and film script format which ordinarily have not been used in works of novelistic fiction. These transgressions from established narrative language draw our attention toward the language and how only a limited potential of the available modalities has been, and is being used by traditional novels, and consequently how another hierarchy of single narrator/point of view has been created based on what kind of tense and writing has to be used in a work of fiction.

\section{The Digital Version}

\section{Physical Organization and Design}

The electronic version of this novel similar to the previous case study has been written by Flash and can be opened in browsers which support HTML. It has been published first by The Iowa Review Web, in its seventh volume of the November edition in 2005. 10:01 is not available on that website anymore and the reader is redirected to Olsen's website as soon as she clicks on the "click to enter" section. ${ }^{29}$ Apart from Olsen's website, the ELO collection is the only other place on the World Wide Web, where this novel can be found.

On the ELO website, this novel has the same title page which has been described in details for Chemical Landscapes Digital Tales. Here the introduction to the novel and the author's description are smaller than the previous novel and the screen capture at the left side depicts a hand grenade in the movie theatre. Due to its web-based presence, this novel has several links to other webpages on the Web, which intensify the dynamic layering of this work that has employed a variety of modes/media in its presentation.

\footnotetext{
29 Although Iowa Review is still being published to this date, April 2012, the Iowa Review Web has been discontinued after the ninth edition at 2009. Also its website has been moved to a new address and that is the reason why the hyperlink at the bottom of the ELO's title page to this novel does not lead to either 10:01 or Iowa Review Web.
} 
The main difference between the title page at ELO's website and Olsen's website is that, the reader in the ELO edition style has to click on the "begin" button to start the reading, whereas the reader is invited to enter the novel on Olsen's website by clicking on the entrance button which invites the reader to enter into the GUI of the novel rather than initiating a process which the word "begin" suggests. ${ }^{30}$ The word "entrance" has this aspect of treating the interface of the novel as a space to go through, rather than the place where the presentation occurs. The keywords for this novel are "audio, collaboration, fiction, flash, visual poetry or narrative." These keywords provide a summary for the materiality of the work by directly foregrounding the modes/media used in this work as well as the software and platform used to create 10:01. The audiovisual elements of this novel work hand in hand with its linguistic elements to present its narrative to the reader.

Before the novel starts there is an intro which moves up on the screen and includes four quotations which, as we will see later, are different from the single quotation by Walt Whitman at the beginning of the print novel, and all of them in one way or another foreshadow Olsen's critique of consumerist society and how the capitalist society commercializes all the mediums of artistic expression. These quotations and their implications will be studied in the narrative strategy section.

The rich interface of the novel appears after this intro, which includes a water-color painting from above and the far end of a movie theatre-that represents a movie theatre in the Mall of America-in an ornamented frame. The start button is right below this painting, and two rows of clickable dots with varying colors are at the bottom of the screen; these rows are the timeline for the novel. In the top section of the painting there is flickering light which represents the unreeling of the movie (or the trailers before the feature film, as is the case here) on the screen. The moviegoers are shown from the rear of the theatre as black two-dimensional heads and shoulders which all of them almost look like each other and have occupied seats in different parts of the theatre. This deliberate similarity of the characters is another physical resource which from the very beginning points to the absence of a hierarchical order here. When all the characters look exactly the same and none of them stands out in the

\footnotetext{
${ }^{30}$ Olsen's website is at: http://www.lanceolsen.com.
} 
crowd, the reader does not have any clues where exactly to look and his gaze is not directed toward any specific point, which again emphasizes that all the characters are on a par here.

By clicking on the start button, the first linguistic section of the novel pops up in the top left of the interface within the frame, and the start button becomes a navigation box with five clickable options. There appears a question mark in the middle, which works as the "help" option in and presents an overview of the interface. According to this overview it is possible to read the novel either by character, character chapters, or by the timeline. Meanwhile a green dot appears on the head of the person whose story has popped up and a big sign on the bottom right of the screen reminds the reader to turn the speaker on. Finally according to this interface, it is possible to hide the text which has covered over the half of the theatre image and click on a single figure in the theatre which becomes orange as soon as the mouse is rolled over it, and his/her name appears at the right side of the navigation box. The space allocated to the linguistic section of the novel in the interface is a little bit more than half of the interface and does not cover the whole interface. This arrangement makes it clear that since the novel has been traditionally a linguistic object, the linguistic section must have a bigger share of the components.

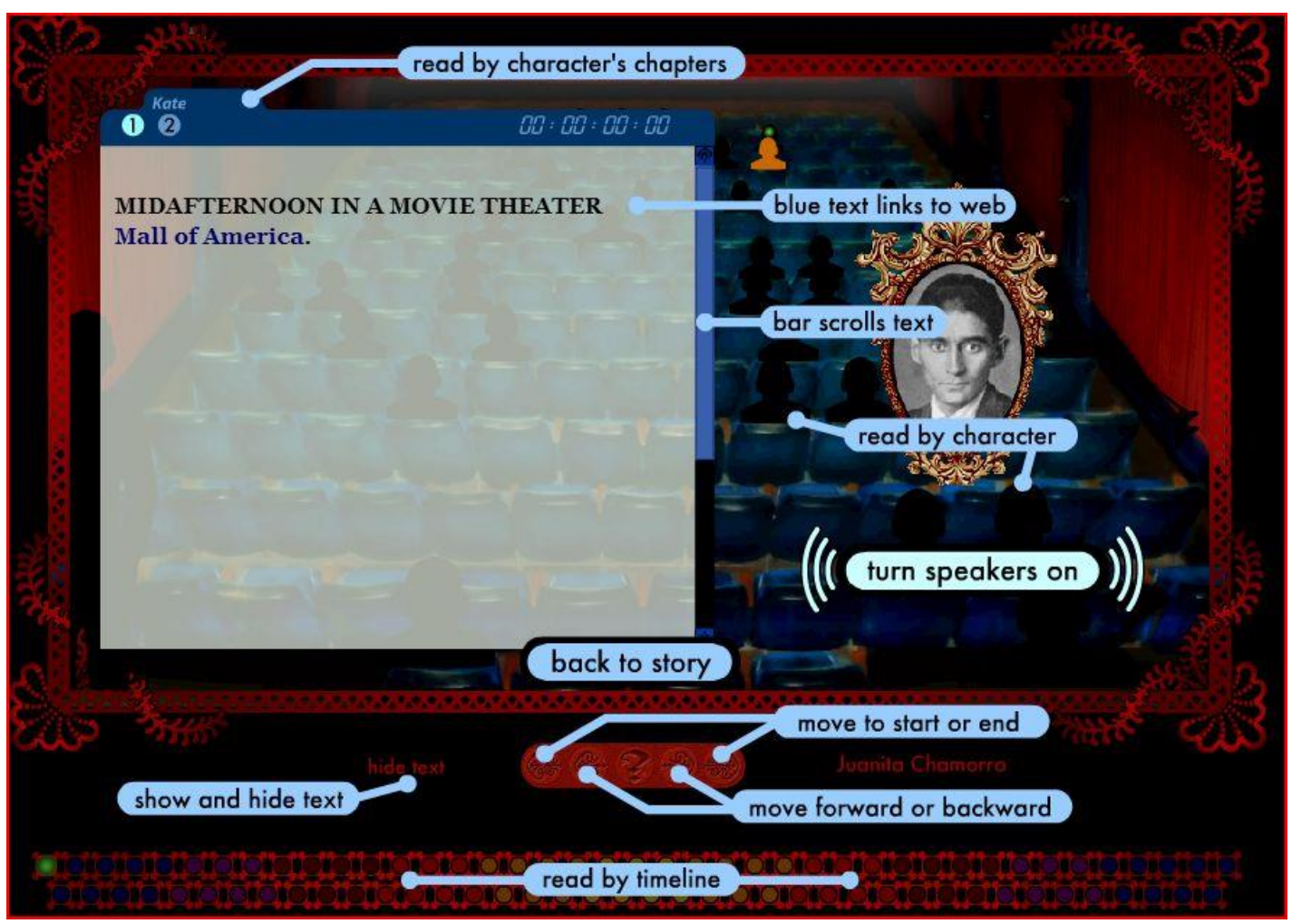


Figure 3.6. Interface of 10:01

The window which pops up for each mini-narrative, in addition to the linguistic section, has the name of the character, one or up to four other circles with numerical digits inside them (which show whether there are any other mini-narratives about this character), and a couple of digits in the stopwatch format, which starts from 00.00.00.00 and ends in 00.09.58.15. Most of these linguistic sections should be scrolled down to be read in full, and they have links to other webpages on the Web, such as the official website of Mall of America, in highlighted blue words. On the right side of this box of linguistic section, a picture or sometimes a movie appears which directly refers to the story narrated in the linguistic section. For instance, for Kate Frazey, who is the great-great granddaughter of Franz Kafka, a picture of the entrance of Mall of America, and a head shot of Kafka appear on the screen each for ten to fifteen seconds, and then disappears. Also for this mini-narrative, and another one about Celan Solen (wordplay on the name of the author), respectively a hare, and a mouse appear on the top of the frame of the painting. The recorded voice and video appear in different parts of the narrative, for instance at 00.00.03:13 when the narrative about Miguel appears on the screen and is accompanied by the sound of deep breathing. Zdravko's narrative has a video at 00.00.04:12 which is a short movie about a woman weaving.

The movie theatre here is also presented through an ornamented frame in order to emphasize the presence of the interface. The frame does not disappear when the mini-narratives of the characters are unraveled, but another frame in a contrasting color is added to it to stress the dynamic layering in this work. The option to hide the text also makes an important claim about the metatextuality of this novel. By hiding the linguistic section of the novel, 10:01 presents itself as a primarily audiovisual work which includes a rich variety of other modes/media such as painting, music, recorded voice, photos, film, etc. Therefore, the interface becomes all that is available to the reader and here Olsen and Guthrie literally create what Drucker had described as "a zone [that] we do not look through it [but] what we read, and [it is] how we read combined through engagement" (9). 
The option to move forward and backward between mini-narratives with the other clickable options near it creates a navigation box for this novel which is more similar to audio/video players for digital files such as JetAudio and VLC Media Player which offer the same options for the audio/video files that are played by them. The multimodal/medial aspect is emphasized by the command "turn the speakers on" which by its iconic sound shape and distinctive white color stands out in the interface.

Another design-related feature of this novel is that in order to mirror the entrance of the characters from a colorful and bright space outside of the theatre into the theatre which is darker and lights are dimmer, the screen lights are dimmed and then get back to normal after five to ten seconds when character move back into the theater. Apart from the obvious links in the linguistic section, there are some hidden links in corners of the frames, (for instance for Stuart Navidson at 00.00.01.01, and Miguel Gonzalez at 00.03.27.29) which are mostly for Date.com in Beaver Bay Minnesota. The relationship of these links to the narrative for which they appear will be studied later in this chapter.

\section{Narrative Strategy}

The narrative of the digital version of novel involves mini-narratives for the 37 people sitting in a movie theatre in the Mall of America. Similar to its namesake, 1001 Nights, the narrative is made of separate (and sometimes interrelated) mini-narratives. These single threads come together in one of final mini-narrative of Milo Magnani, the assistant manager of the mall, at 09.58.15, which has the longest mini-narrative and can get into the minds of the other characters in the novel and reveal to us what they think. He even knows what happens in the future for a few of them.

As stated in the previous section, before the mini-narratives appear on the screen, there is an intro which includes four quotations. The first quotation is a statement about the movies by August Lumière in 1895. According to Lumiere: "Film can be exploited for a certain time as a scientific curiosity, but apart from that it has no commercial value whatsoever." This quotation dates back to the same year that Lumiere brothers showed the first projected movie to a paying audience. This quotation describes film and the reasons for seeing it as a scientific curiosity. The 
main assumption here is that similar to the prediction about digital fiction, the interest would be lost once curiosity was satisfied. However, the movies proved him wrong. At the time of the invention of the film, he would not have been able to see the huge potential of the movies as generating huge commercial interest and their longevity within the culture.

The second quotation is a dialogue from George Romero's Dawn of the Dead which is uttered when the zombies keep coming back to a deserted mall where the main characters have taken shelter on its roof:

"Francine: What are they doing? Why do they come here?

Stephen: Some kind of instinct. Memory of what they used to do. This was an important place in their lives."

This quotation refers to how shopping has become a central part of our culture and how the malls include almost everything now. The Mall of America is a good example here because it includes a theme park, several food courts, a sea life aquarium, an upscale hotel and fourteen movie theaters. At the time of this writing, there are plans for expansion of this mall to include a dinner theatre, ice rink, three hotels, and a waterpark. These expansions will double-up the size of an already huge mall (currently over $452,000 \mathrm{~m}^{2}$ ) which will make it enough to fit fourteen Yankees Stadium inside it. In this regard, a mall becomes more similar to the digitality which has become a huge part of our culture and is expanding more and more each day.

The third quotation is from Letters to Wendy's (2005), a book of experimental essays/letters by the contemporary poet and writer, Joe Wenderoth:

Let's say Wendy's is an airplane. Traveling at ten thousand feet. Let's say there is no landing gear and nowhere to land. And fuel is limited. And one has a general idea of when the fuel is going to run out. Given this knowledge, is travel really the right word? And if not travel, then what? One sees one's life quite differently when one knows it isn't going to land. (ELO Website) 
This quotation in this context can be interpreted as an indirect reference to the digital materiality of the novels and how the digital novels by keeping at bay the certainties which the traditional works of print fiction offered-for instance a definite place to start, a definitive end and a reading method which did not change from novel to novel- have changed the way the novelistic fiction has been seen, and brought about a different understanding of the novel. Though the quotation does not directly address what these differences are, interpreting it in this context makes it possible to think of those differences as paying attention to the materiality of the medium and how it affects what is being presented through it.

Finally, the last quotation is from a David Bowie's single 'I'm Afraid of Americans" and simply says: "God is an American." Before analyzing this quotation, let's have a look of what the singer/composer has to say about it:

It's not as truly hostile about Americans ... it's merely sardonic. The invasion by any homogenized culture is so depressing, the erection of another Disney World in, say, Umbria, Italy, more so. It strangles the indigenous culture and narrows expression of life. (Earthling Press release)

So here the Bowie quotation becomes more similar to the issues discussed in this thesis, in the sense that it aims to resist the totalizing effects that an order/tradition has on the arts (expression of life). The way that the physical resources of the book had been used in traditional novels is a good example of this invasion of homogenized culture, and that was exactly what the metatextual novels were resisting. Digital fiction, by following experimentations of metatextual novels, can offer great opportunities for those writers who want to foreground the materiality of their work.

Breaking the narrative into smaller chunks, or mini-narratives as they are referred to here, makes the narrative similar to the works of hypertext fiction, but this is also where the similarity ends, because in contrast to works like afternoon, the reader has access to all the parts of the novel from the beginning and the mini-narratives are not directly related to each other, in the sense that the lexias in the works of hypertext were. This independent status of the mini-narratives makes the controlling mechanisms such as guard-fields unnecessary too. These mini-narratives 
(or tales) can follow each other in a variety of ways, and the temporal arrangement of the mini-narratives which move forward in time is only one possible option here. These mini-narratives, similarly to the "tales" of the previous case study, make it possible for the novel to have a variety of stories, instead of a single one. In other words, the narratives here are woven out of a variety of threads and the interface of the novel allows several narratives to exist together and the reader as can be seen below can choose which one to follow and explore each of them in various ways.

\section{Reading Process}

From the beginning there are three ways to construct the narrative discourse of this novel, and each one will unravel the narrative in a different way. The first one which is referred to as "read by timeline" in the interface of the novel, as its name states, follows the traditional method of reading the story based on the time progression within the story. In this process, what the reader has to do is clicking on the forward button after the first mini-narrative appears. For this reading all of the mini-narratives are read sequentially and one after the other in the same way that print version of the novel is expected to be read. Here the reader starts with Kate's mini-narratives at 00.00.00 and moves linearly in time forward until she reaches the mini-narrative named "begin" which is when the movie is supposed to start and clicking on that mini-narrative takes the reader back to the beginning of the novel as well.

The second way of constructing the narrative discourse in this novel (which is referred to as "read by character chapter's" in the interface) starts from the traditional method and follows the story of the characters which seem more interesting to each reader by clicking on the next mini-narrative for this character in the circles, and visiting the other mini-narratives which are about him/her. This method gives more options to the reader because he can read those which interest him more and ignore those other characters which he does not want to follow. Unlike the previous reading process, this one is not necessarily chronological and moves back and forth in time.

The third way of constructing the narrative is referred to as "read by character" in the interface and as the name suggests requires the reader to click on where every character is sitting and follow his/her narrative from there. This kind of 
reading has the least authorial control over the act of reading, provides the reader the opportunity to create a specific version of the narrative discourse of the novel as a result of the reader's specific acts of selection. The main difference between this reading and the previous one is that in the previous one the reader was supposed to read all the other mini-narratives which existed in the text window too. There could be up to five mini-narratives for a single character. However, in this reading the reader is supposed to jump around and read one single mini-narrative from each character and move on to the next character. The other mini-narratives for that character can be read if the reader gets back to him/her later in her reading.

The last possible reading process is mixing all of these reading processes. So for this one a reader can start from a character, read a couple of his mini-narratives, then move to another character and from there on read a few by the time line. Any of these different reading processes can potentially highlight the roles of different characters within the story. By not providing a single or fixed narrative discourse the author here is giving readers the freedom to choose their own way through the pool of stories. One of the implications of this equality is that the Web has become like that theatre itself. It provides a space in which different media/modes such as typographic elements, picture, voice, video and music can come together to form a narrative. This multimodal digital space can be exploited to create narratives which are similarly organized around time, but meanwhile it provides other opportunities for the readers to create their own narrative discourse and reading paths. This point is stressed from the very beginning by the interface of the novel which does not show the screen of the theatre, but the people who are watching that screen. In fact, the text box which opens for different characters can be considered as a displaced screen which has replaced moving images with words and in doing that has magnified the importance of the written language as the major element in works of multimodal fiction.

So as can be seen through displacing the movie screen with the text box, the materiality of the medium is stressed here from the very beginning. While watching a movie the audience are meant to get absorbed in the experience of watching that movie, not the movie theatre and the other audience, but in 10:01 the writer wants us to see the audience within the movie theatre itself. After all, almost all movies are about the life of other people, (how do they fall in love, how do they overcome their 
problems, how do they survive in a post-apocalyptic world invaded by aliens, etc.), and this is exactly what we miss when we go to watch movie and share the experience of watching a movie with strangers, but cannot know what their stories are. Here the medium helps us to have a better grasp of who and where these people are, what they think and how they interact with each other at a specific moment at this specific place. Thus in this novel we can see the movies inside their head whether they are memories or actual movie which they have actually made or wish to make. One of these movies, Where the smiling ends, makes the point quite well. This movie is about the moments immediately following the time people photograph each other,

when people slowly stop smiling after the shot has been snapped and you can actually see their public mask soften and melt back into everyday blandness, a gesture always accompanied by a slight lowering of the head in a miniature act of capitulation. (ELO Website)

This short experimental movie, which actually has been made by Lance Olsen's partner, assemblage artist Andi Olsen, makes the audience pay attention to something which they normally do not see or pay attention to in their daily life. But this moment too, like the moment before that, is a part of the daily life of the people. The moment before this one, the moment which their picture is being snapped by the camera, is the one which is immortalized through the effect of light on the chemicals, but that photo and that smile is a momentary reflection of the people toward the camera, which is supposed to fix this moment, and they get so much absorbed in the moment their photo is being taken that they do not pay much attention to what people do after that.

Another interpretation of this short movie is that fiction provides a focus on aspects of social life which people actually engaged in social life do not have to think about, and at the same time it points to the role of technologies of representation, and by implication how they have become increasingly popularized. Therefore, whereas previously 'taking/making' a picture required expensive technology and expertise, now it does not and so a previously very small section of the population, who learned how to pose for their portraits, has been replaced by a much broader section of the population practicing public/celebrity personas, that is, engaged in specific kinds of 
(self) representation which conform to public forms of expression in relation to specific media which includes digital media as well.

\section{Print Version}

\section{Textual Organization and Design}

The print version of 10:01 has been published by Chiasmus Press, an independent publication house in Portland, and the book has been designed by Matthew Warren. The cover is a blurred photo by Mia De Bono of the back of a man's head looking at a yellow shiny circle in the top left side of the cover. Although the quality of the picture is not very good in the print cover, looking at the cover on the author's official website and other sites such as Amazon.com make it clear that the shiny object is the sun, and its relevance to the novel will be analyzed later.

In keeping with the temporal arrangement of its mini-narratives which has a time signature at the top of each mini-narrative, the next section of the book which is the dedication page, is a dedicated to Andi, Olsen's Partner, "27:07:365." The following page has a quotation by Walt Whitman which reads "you must habit yourself to the dazzle of the light." This quotation provides a good clue for what the cover is about and will be analyzed in the narrative strategy.

Similar to the digital version, the mini-narratives of the novel start at 00:00:00:00 and end by the final one which starts at 00:09:54:27 and presumably ends at 00:10:01:00. However, the number of mini-narratives is quite larger here and includes over a hundred mini-narratives which vary in length and therefore do not occupy the same page space and some of them have only one or two lines under the time signature.

One feature of this novel which makes it look different from the other novels is that in trying to mirror the diversity of the voices and the characters in the novel, it has tried to use different kinds of presentation of the words on the paper. For instance, in one section about Celan Solen, the text becomes a short movie script for a movie about Mona (Celan's girlfriend) and Mosh (Mona's beloved). Another section which is about Cynthia Morgenstern has numbered sentences. In another one, about Juanita 
some of the sentences have been presented in the boldface type in order to show the trend of thoughts inside the head, or the mind's eye of the character.

The three outstanding features of the graphic surface of the print version of the novel are the time signatures, displaced page numbers and the half-blank pages. The first two features are closely related to each other because of the capability of each one to override the other. They both start when the first mini-narrative of the novel begins a few pages into the book, and they both end on the page that the last mini-narrative ends. Page numbers have been traditionally the only numerical mark which appears consistently at the very top or very bottom of the print page. Here, this numerical mark finds another numerical companion which is bigger, larger and appears on almost every page as well. These time signatures are also more integrated into the narrative because they have a crucial role in informing the readers about the time in which each mini-narrative is being unfolded. On the other hand, the page numbers seem to be a redundant part because their rival makes it possible to find and cite each mini-narrative in the same way that they have been cited here. The result of this tension between the page numbers and time signatures is that one of the ordinarily transparent features of the print book, that is, page numbers, is brought to the foreground and its raison d'etre is compromised, and in doing that the graphic surface of each page is foregrounded.

A reason for the presence of half-blank pages in this novel is that the graphic surfaces of empty spaces on those pages draw attention to themselves and the page becomes a spatial unit, on which the words can be arranged in different ways. In other words, instead of following the arrangement of words in the traditional print novels - which through similarity of the arrangement of the words in each page, and normalizing them, intend to make the body of the book invisible-the pages in this novel, by disrupting that order create a spatial unit which does not intend to erase itself. So it can be said that similar to the changes in fonts and word arrangements in the other metatextual novels such as Willie Masters' Lonesome Wife, distinct arrangement of the words on every page also shows the diversity of the characters which the novel intends to depict. 
The white space on the page also carries forward the light, and the black print carries the shadow. This interpretation becomes justified when it is analyzed in the context of the photo of the sun on the cover of the book, and the Whitman quotation in the opening pages of the novel. The Whitman quotation "you must habit yourself to the dazzle of the light" has been taken from his famous poem "song of myself" and in that poem Whitman addresses a person who has just woken up both literally and metaphorically, from his dreams and is now experiencing the dazzling light. In the context of this novel, the quotation from Whitman can be related to Plato's cave, where we see only shadows because the light source is not directly accessible. When someone moves out of a movie theater into a bright day, the dazzle of the sun might be blinding to her but she should habit herself to that dazzle because what the movies show is just a representation of a fragment of the life of the people. Likewise what is presented in the novel as its narrative is just a representation of fragment of the life of the characters and the white pages remind the readers that a large part of their life has been left unsaid. As Celan Solen once says "life flies at us in bright splinters. We turn them into significance" (42), each mini-narrative just tells us a splinter's worth of the whole life of any of the characters. In this respect the black shadow becomes broken into signs, i.e. distinguished from each other and hence able to signify, by the white space in which they are contained. This quotation also directly connects with the linguistic section of the Chemical Landscapes which fly to us and we can only read a portion of them before they disappear and then we turn these portions/splinters into significance.

\section{Narrative Strategy}

The 105 mini narratives of the book are by the fifty two people who are sharing a theatre in Mall of America. Although these characters share the same space in the theatre, they do not have the same share of narrative in 10:01. Some of the characters such as Max Watt, show up five times in those mini-narratives, and others such as Claude and Mouche Melies appear only once. Most of the mini-narratives are about one single character, and there is only one with three characters. This mini-narrative which is about Lakeesha Johnson and her friends, starts with Lakeesha's fake phone conversation, and then moves on to her friends, but they disappear in the next mini-narrative at 00:06:09:10 which is solely about Lakeesha. 
Her mini-narrative is narrated by an omniscient narrator who can penetrate into the minds and feelings of the characters and narrate them.

Although mini-narratives in the book have varying lengths, the changes in time signature are not directly related to the length of these narratives. For example, the time signature for the mini-narratives jumps only one second ahead for the first nine mini-narratives, but it suddenly jumps from 00:00:09:09 to 00:02:53:01 in the tenth one where/when Byron Metnick, the scrawny usher finds a "cute little blind girl" lost in movie theatre. In this mini-narrative Byron takes her hand and leads her to the lobby to her parents, who are relieved to see their daughter again, and then gets back to the theatre.

The other part where different mini-narratives come together under a single time signature is at 00:08:35:06 when Angelica reaches out for Miguel's hand, and Miguel pushes her hand away, also at the same time Kosa, the ghost of Zdravko's wife, passes through Zdravko's head and makes him dream about her. Meanwhile a cat gets back to the aisles and into what is described as "insanity of legs". In the first mini-narrative of this section we read about Zdravko's dream of a woman in Omarska's camp who is cleaning the torture room, and then suddenly he recoils from this dream as a result of Kosa's ghosts passing through her mind. Although the time signature is fixed here, their sequential arrangement presents one before the other.

As it was said earlier, different modes of language have been used in this novel to show the diversity of the people and opinions that they hold here. For instance, there is an imaginary interview with an African American teenager, and the questions are omitted but the reader has to infer what the questions have been through the answers of the character. There is also a mini-narrative for Cynthia Morgenstern in which each sentence has a number. As the above examples show print fiction can include a variety of writing modes: interview, list, film script, etc.

The multiple characters in this novel represent the diversity of the society in which people live. This diversity is worked out through the verbal idiosyncrasies of the characters. This includes the dialect and idiolects of the people who use the same language, but in a totally different style. It is through these linguistic representations that the cultural background of the characters is also revealed. Although this feature 
exists in the digital version of the novel as well, since other modes such as movie, image, music, recorded voice, and so forth are absent in the print version, printed language becomes a major mode for the representation of the characters in the print book. Therefore, these verbal idiosyncrasies become more significant and help identify which character is saying what, even if his/her name is not mentioned.

This linguistic representation is not limited to the dialogues only, but exposes itself in the whole section of the mini-narratives. For instance, in order to show the African American background of Lakeesha Johnson, the narrative languages changes to African American Slang: "Some wack shit is going down up there involving a skinny-assed white boy in some phat-up silks dangling off the bottom of a train" (56). In another part of the novel, the slang of the Kentucky farmers is represented: "That's where he done had his first visitation one night out harvesting all by his lonesome. Bright triangle appears outta nowheres and them voices start going at it in his head hens in a chicken coop Johnny Ray all dizzy like as if he's walking on a slant" (56).

\section{Reading process:}

The main reading process for this work is to follow the chronological order and sequence in which they are presented. Unlike the digital version, there are no alternative reading processes here. Obviously the reader can jump around and read random time signatures, but the way the book has presented them provides only one expected reading. Because of the same single expected reading process, the number of mini-narratives is significantly larger than the digital version. The alternative reading paths in the digital version exponentially increased the possibilities of constructing the narrative discourse there. However, despite this forward progression, because of the large number of characters, the reader might not be able to keep track of all of them, and she might return to the first few pages of the novel to re-visit the previous mini-narratives for some of the characters. In this way, the reading process here becomes cyclical. 


\section{Conclusion:}

Despite their similarities, the two versions of 10:01 offer different experiences of reading and this is the direct result of their different instantiation. Each novel has tried to take advantage of the materiality of its medium and the text for each version has been changed accordingly. For instance the linguistic section of the novel has been shortened in the digital version in order to make room for the other modes in its presentation. On the other hand, the print version had highlighted its graphic surface by placing the time signatures at the top of the pages and leaving almost half of many pages empty. Although these changes in the arrangement of the words on the pages are not as radical as the print novels which will be studied in the next chapter, their presence in the print novel shows that print has achieved a media consciousness which can be seen in the works of many novelists. What this media consciousness is and how metatextual print novelists have responded to the digital phase of the novel will be studied in the next chapter. 


\section{Chapter Four: Metatextual Print Fiction in the Age of Digital Fiction}

Almost all contemporary literature is already digital. Except for a handful of books produced by fine letter presses, print literature consists of digital files throughout most of its existence. The digital leaves its mark on print in new capabilities for innovative typography, new aesthetics for book design and in the near future in new modes of marketing. (Hayles, Electronic Literature 159) 


\section{Introduction: "The Mark of Digital"}

One of the most visible effects of digital technology on print fiction is the way that it has facilitated more diverse forms of writing, editing, pagination and other technical issues involved in writing and publishing a work of print fiction. However the main question is, how and in what ways has the introduction of digital media as an alternative material support for the novel affected the development of metatextual print novels?

As it was seen in the previous chapters, the materiality of the medium does play an important role in the signification process; Gass and the hypertext fiction and web-fiction novelists have incorporated the materiality of the medium in their signifying strategies. Therefore, it is worth exploring how metatextual print novelists in the late 1990s and the first decade of the new millennium have foregrounded the materiality of print and its physical resources in their works, and how similar and different are their efforts from their print and digital counterparts, particularly Willie Masters' Lonesome Wife which was written and published before the digital media became available.

The most direct effect of digital media on print was the way print remediated the digital media forms in various ways and inspired metatextual novelist to blend the physical resources of the print media with their signifying strategies in ways which some metatextual novelists before them (for instance, Gass) had used. This remediation resulted in novels with more "visibility" (Hayles and Gibbons) in the post digital phase. What both Hayles and Gibbons mean by "visibility" is, in the terms used in this thesis, the highlighted and foregrounded graphic surface of these novels which one of its direct results is that it makes the materiality of the text more visible. In other words, the outstanding metatextual features of these novels are evident to anyone who would pick them up from the shelves of a bookstore and leaf through them.

For the case studies of this chapter I have chosen works by two authors-Bantock and Danielewski-who are very different from each other in how they explore the possibilities of fictional narration in the print medium, but each one shows a different development in the way the digital-era metatextual print novelists 
have reacted to novel's going through the digital phase. Nick Bantock's works which have been published and marketed by Harper Collins, one of the world's largest publishing companies, provide the reader with a mixture of images and words and he tries to marry words and image together so that they can have an equal role in creating the novelistic fiction. Mark Z. Danielewski does not use images as extensively as Bantock, but he also pushes the boundaries of the book as a medium to new extremes and creates narratives which take advantage of their material support in a variety of ways. These case studies will show how both of these writers explore the potentials of fiction, and how different/similar their works are.

A prominent characteristic of the digital-era metatextual novels is the concept of dynamic layering which was explored in the previous chapter. This chapter will not delve into the technical know-how of how a print novel is created from a digital file. Nevertheless it should be emphasized that these novels are "born digital"31 as Hayles has demonstrated and argued that print "should properly be considered a particular form of output for digital files rather than a medium separate from digital instantiation" (Electronic Literature 159). What will be analyzed here instead is the way this layering is created in works in contemporary metatextual novels and how these novels foreground their graphic surface and emphasize the presence of their physical body in the print medium.

\section{The Print Media Revisited}

As has been discussed from the beginning of this thesis, metatextual novelists have consciously highlighted the role of material features of print in their novels since the eras known as high modernism/early postmodernism in literature. But other than Kaufmann's and White's writings, from whom this thesis has borrowed its terminology, I have not been able to find scholarly works which particularly focus on this aspect of the novels in the last fifty years. The absence of a significant body of critical writing in this specific field, has led Alison Gibbons, one of the new scholars in the field to state in her book, Multimodality, Cognition, and Experimental Literature (2012) that

\footnotetext{
${ }^{31}$ Or as Joseph Tabbi has put it, this birth/migration is a "nomadic meandering back and forth across a boundary" (Tabbi, 119).
} 
One might expect that as writers have been experimenting with the inclusion of images in narrative..., a critical vocabulary for examining such experimentation and design would be established. Regrettably, this is not the case. Inevitably, literary criticism has instead favored the word, and often not as the written word, focusing instead upon the thematic and conceptual facilities of discourse. (1)

Gibbons' own effort to contribute to this neglected field is to bring those experimentations under the umbrella term of "multimodal literature" and create a context for them in the way that she defines them. According to her, multimodal literature is

a body of literary texts that feature a multitude of semiotic modes in the communication and progression of their narratives. Such works are composed not only of words,...[they also] experiment with possibilities of the book form, playing with the graphic dimension of text, incorporating images, and testing the limits of the book as a physical and tactile object. ( Bray, Gibbons, and McHale 420)

These features as she goes on to describe have become cheaper and easier to produce due to the involvement of digital technologies for book publication (or the "mark of the digital" as Hayles would have it), and that has been the main factor in the increase of multimodal works in the recent times. What Gibbons totally downgrades in her analysis is the simile that Hallet had used a few years earlier, in an article aimed at "introduc[ing] the genre [of multimodality] to narratologists." According to Gibbons, Hallet "likens reading a multimodal novel to reading a hypertext" (ibid. 421), a comparison Gibbons refutes by saying that "comparisons to alternate media are (almost inevitably) generalizing" (ibid.). However, as it is shown in this chapter, these novels, whether one calls them multimodal novels, or digital-era metatextual print novels, should be studied in the "media ecology" of their own time. The recent increase in these novels (and the scholarly work which has finally started to appear, spearheaded by the invaluable works of Gibbons herself), apart from the cheaper methods of book production - which helped to alleviate the cost-effectiveness constraint for the experimental print writers to a large extent - can be the result of the 
broader media consciousness which appeared after the digital media phase of the novel. As one can easily observe by reading the essays in the Routledge Companion to Experimental Literature-edited by Bray, Gibbons and McHales,- - experimentation has existed alongside the traditional novel from its beginning, and novelists have experimented with all the features of the novel and the book throughout the not-so-long history of the novel. However, what distinguishes a book like Willie Masters' Lonesome Wife from its contemporary equivalents such as The House of Leaves in terms of conception, structuring, and reception, is the same contemporary media-consciousness and the dynamic layering which has ensued from the involvement of an "alternate media" as a material support for the novel. Before this digital phase, experimentation with the physical body of the novel is interpreted by one writer (Gass) as a failure, and those experiments are abandoned in his subsequent novels such as The Tunnel (1996). However, after this digital phase, experimentation with the materiality of the book for another writer (Danielewski) becomes fundamental to his success as innovator on and he continues to explore in the same direction in his works.

On the other hand, what Hallet has said is more meaningful in the context of this media-consciousness which was highlighted after the digital phase of the novel. Although Hallet does not specifically name works of hypertext fiction as works that their experience of reading is similar to reading multimodal novels, he explicitly acknowledges the similarity and states that

to a certain extent, the reader's activities start to resemble those of the user of an electronic hypertext (although in most cases the multimodal novel is a linear narrative, whereas the electronic hypertext is a non-linear ensemble of texts and signs)" (qtd. In Bray, Gibbons, and McHale 421).

The linearity of "most cases [of] the multimodal novel" is open to investigation, but whether Hallet is right or not about it is beside the point because he correctly does not include linearity or non-linearity/multi-linearity as one of the features of multimodal novels. Although the case studies here are multimodal, they are not necessarily linear or non-linear. In fact the linear narrative of Bantock in his 
multimodal novel becomes one of the features which distinguishes his novel from Danielewski's non-linear narratives. But, there is something else in this comparison which Gibbons anchors her critique of Hallet on. As can be seen from Hallet's quotation, his term for the person who reads an electronic hypertext is "user" which has become an established term to refer to the person who engages with machine interactions. Although other critics have suggested alternative terms for it, such as interactor (Murray 1997), and subject (Drucker 2011), none of these terms has become popular and nowadays words such as username, graphical user interface, and user-friendly have become the standard terminology in the digital world. So by saying users of hypertext, one is actually referring to the natural terminology of the field and this term does not have a qualitative connotation in this usage. However, Gibbons takes this for granted and goes on to say that "we might want to think of reading multimodal novels in active terms: not just as using, but also as engaging and performing" (Bray, Gibbons, and McHale, 421). As discussed above, Gibbons' refutation of Hallet's claim is based on her different understanding of the word "user" which in reading a work of hypertext fiction is anything but passive, because as it was seen in the second and third chapter, at each point a user has to make choices and move to different lexias/pages regardless of whether she is reading a literary or non-literary hypertext or web-fiction.

Hallet's point, on the other hand, can be better understood when it is placed alongside Hayles' discussion of House of Leaves in her book Writing Machines (2002) where she identifies Danielewski's novel as a print hypertext. Hayles' choice of terms shows the mutual effects of print and electronic textuality on each other and how reading a metatextual and multimodal print novel can be similar to the experience that a reader has while reading a work of hypertext (or web)fiction. The major implication of Hayles' argument is that these novels are the print instantiations of files born in digital media, which as Lev Manovich (2003) has pointed out contains all other media within itself. Therefore, the multimodality of the House of Leaves and other novels which Gibbons names is directly related to their digital birth. Even writers, like Danielewski, who insisted on using pen(cil) and paper in writing their novels, could not get away from using a computer at some stage during the production of the text. For instance, Danielewski in his interview with McCaffery and Gegrory 
emphasized that he had written the whole novel with a pencil, but later in the interview he admits that he spent a whole month in New York to create the digital file for it so that it could be published by Pantheon. Despite his insistence in that interview on using a pencil, he eventually surrenders to the "mark of digital" and in an interview (2012) with Kellogg from L.A. Times he states that he is currently using a writing program (Adobe InDesign) for writing his novels. So the digital birth of the contemporary novels (both traditional and metatextual) is becoming more and more common. Therefore, though this digital birth alone does not automatically make a novel metatextual, it facilitates the media consciousness of the metatextual writers and motivates a wide variety of responses from these writers. Looking at the argument in this way will provide a better reason and a more useful background for the increase of metatextual novels, than just the cost-effectiveness of the new digital media for the book publication industry which Gibbons identifies in her book.

\section{Mediums Under Pressure: Print and Painting}

Print has not been the only medium which has been challenged by another medium. A useful comparison for the way another medium/art form has responded to the challenges of a rival medium is the way painting responded to the challenges of photography. From the mid to late nineteenth century, photography underwent major developments and challenged painting as the only art which could provide "an accurate record of the observable world." As a result several art movements such as Impressionism, Post-Impressionism, Fauvism, Expressionism, Cubism and Dadaism, were formed in the twentieth century. One of the landmarks of these movements was Abstract Expressionism, which totally moved away from the referential role of paint and draw attention to the materiality of the elements which created a work of art. These elements were not used to represent an external reality, but to create an independent entity in this world. Elements such as color which in the past served a referential role, now had become "a completed presence" in a work of art. So it can be said that painting changed through the introduction of a rival medium, and the transparency in representation which it was aiming for was replaced by opaqueness brought about by foregrounding the materials which created that work of art. 
Although we have not yet witnessed the surge of literary schools from the challenges which electronic media has brought to the novel equivalent to what happened in painting, the same parallel process can be identified in the relationship between print and electronic media. A good example for this is the way print writers are advised by experimental writer Garth Risk Hallberg, author of A Field Guide to the North American Family (2007), in The Millions website, in 2011, to kindle-proof their works by following seven instructions which include using color, illustration, typography etc. ${ }^{32}$ Hallberg's advice shows how more and more writers are being drawn toward creating novels which use a variety of modes and at the same time take advantage of the unique affordances of the book. Any of those seven instructions in the hands of a writer who pays particular attention to the materiality of her work will be used in order to draw attention to aspects of that materiality and create a metatextual novel. Although Hallberg's main point is that Kindle cannot reproduce these features of print, so it is advisable to use them in order to make it impossible to port your novel to Kindle, the ways that he recommends as well as the list of ten Kindle-proof novels that he provides at the end of that article have the common theme of generating more media consciousness for the reader/writers whom Hallberg is addressing. So it is not surprising that those ways are similar to the different categorizations of multimodal novels that Gibbons makes in her essay in Experimental Literature because both of them are discussing what can be better described as the result of media-consciousness of experimental print fiction in the digital era which generates metatextual novels.

\section{Digital-era Metatextual Print Novels; Toward a Definition}

In her discussion of the digital-era experimental print novels, Hayles identifies

Two apparently opposed but actually complementary strategies: imitating electronic textuality through comparable devices in print, many of which depend on digitality to be cost effective or even possible; and intensifying the specific traditions of print, in effect

\footnotetext{
32 The seven steps that he recommends are: 1.Use color 2. Illustrate 3. Play with text, typeface, and white space, 4. Run with scissors, 5. Go aleatory, 6. Put it in a box, 7. Pile on the end matter. His essay can be found here:

http://www.themillions.com/2011/05/kindle-proof-your-book-in-seven-easy-steps.html
} 
declaring allegiance to print regardless of the availability of other media. Recursively entwined, the two strategies often appear together in the same text. Moreover, they tend to morph into one another. (Hayles, Electronic Literature 162)

These strategies that Hayles has identified provide us with a sound basis for understanding what has happened to the experimental novel after the initial digital phase. Informative as Hayles' discussion is, her argument here becomes hard to follow for the metatextual novel because of the morphosis of these two strategies and their co-presence with a single novel. There is no denying that these two do morph and do appear within one text, but even Hayles cannot push her argument further when she analyzes three sample novels according to her own criteria and she has to bring another yardstick, the four characteristics of the electronic texts, to explore the digitality of those novels. Three out of those four characteristics (layering, multimodality, and separation between storage and performance) have been discussed in detail in the previous chapter and her fourth characteristic, which is the time-based nature of the electronic texts, has been discussed from another perspective, because it does exist in a work like Chemical Landscapes Digital Tales; but other works such as 10:01 do not have that characteristic as Hayles has defined it.

On the other hand, in the ensuing pages she adds other features to this list, such as mutability, that are not initially mentioned. Finally her discussion is for digital text in general and though she goes on to focus on print fiction in her examples, her discussion could have been more closely related to this thesis if it had focused on digital fiction from the beginning. So here I return to the way digital fiction has been defined in order to explore the potential for analyzing the ways in which digital-era metatextual print fiction draws upon the new potentials for fiction opened by the writers of digital fiction discussed in the previous chapter.

As we saw in the previous chapter, Bell et al. had defined digital fiction as "fiction written for and read on a computer screen that pursues its verbal, discursive and/or conceptual complexity through the digital medium and would lose something of its aesthetic and semiotic function if it were removed from that medium" (Bell et al. 2010). It is possible to repurpose this definition for the digital-era metatextual print 
novel by changing it thus: fiction written for print that pursues its verbal, linguistic and/or conceptual complexity through the print medium and would lose the way its physical resources are aligned to its signifying strategies if it were removed from that medium. Apart from the features discussed above, non-transferability becomes an important feature of this phase too and when Hallberg advises writers to kindle-proof their novels, he is directly advising writers to make their works non-transferable. However, this non-transferability refers to specific aspects of print materiality which cannot be replicated in a digital copy. For Hallberg, digital materiality is a threat and the way to overcome that threat is to make your work as non-transferable as possible, but some other writers like Danielewski, whose novel has almost all of the features that Hallberg has recommended, have reacted differently to this digital materiality. Danielewski found an ally in the digital medium and realized the potential to expand his works into these media. He set up a forum on his own personal website for House of Leaves (and later his other novels) ${ }^{33}$, and his sister created the music album Haunted which was released in the same year that House of Leaves was published. In order to extend this multimediality to print, Danielewski published Whalestoe Letters (2000) as well to show how this multimediality can include print in its media ecology as well. Therefore, Danielewski deliberately expanded his work in the digital media and took advantage of the affordances of each medium to create various layers of interpretation.

\footnotetext{
${ }^{33}$ http://forums.markzdanielewski.com/
} 


\section{Case Study No.4.1: The Forgetting Room}

\section{Introduction}

Under the best circumstances, my books emerge out of a number of seeds - a scrap of image, a few words strung together. The sentences suggest other images, and the scraps lead to paragraphs....It's not a matter of illustrating a story, nor is it a matter of words annotating pictures. The balance needs to be maintained from the start....For me images and text work together to unblock one another, just as I hope that the integration of words and pictures leads the viewer toward a liberated sense of perception. When word and picture marry, the left and the right sides of the brain operate simultaneously, and a means of expression is available that offers far more than the limited view of existence we have become used to. The union provides a meeting ground where our two primary methods of observing reality can coexist. (Nick Bantock, Artful Dodger 216)

Although Bantock comes from a painting discipline, he does not favor images over words and believes that word and image should have a mutually interactive relationship. As he claims in the above quotation there is a parallel between the interaction of word and image, and a psychological event. The word "unblock" meaning releasing something which is caught or trapped has been used here to show that the potential for narrative of other dimensions of the textual organization and body, which have been suppressed and ignored by the traditions of print in the past, could be "liberated" by using images which neither aim to annotate nor illustrate the words which accompany them. The co-existence of these "two primary methods of observing reality" creates a multimodal work that activates both hemispheres of the brain and results in a work which "offers more than the limited view of existence we have become used to" (ibid.).

True to his word, Bantock from his very first novel trilogy, Griffin and Sabine (1991) has been creating works which marry words and images in order to liberate the 
viewer/reader's perception. What Bantock means by this liberation is better understood in the light of the relation of language to brain hemispheres and the scientific studies of brain anatomy and its relation to the humanities and history of the ideas which Bantock hints at in one of his interviews. The work which Bantock refers to is The Alphabet Versus the Goddess (1990) by Leonard Shlain, follows this theme:

...with the advent of literacy, the very act of reading an alphabet reinforced the brain's left hemisphere-linear, abstract, predominantly masculine at the expense of the right holistic, concrete, visual, feminine. This shift upset the balance between men and women, and initiated the disappearance of goddesses, the abhorrence of images, the decline of women's social and political status, and a long reign of patriarchy and misogyny. (back cover)

In other words, Bantock's aim is to restore the balance in both hemispheres so that consequently the reader/viewer sees this relationship with a new perception. Although Bantock does not specifically uses the words modes or multimodality, he is supporting and creating a rationale for the co-existence of these modes in a work of fiction. What this co-existence entails is bringing the design features to the foreground, because as soon as another mode walks in and unsettles the long reign of the other one in print, the design becomes an important feature to consider. Where should the images on the page be? What percentage of the book should be allocated to them? What kind of relationship to the narrative should they have?

So it can be concluded that Bantock's theory of art looks to the creation of objects in which design plays an equally important role as the other elements which conventionally have formed the basis of an artwork. In the case of novels, he foregrounds the way the non-linguistic elements open up a dynamic relationship with the words, and at the same time highlights how bringing this feature into play can be beneficial for liberating the human mind from the traditions which have shaped the conventional form of the novel.

On the other hand, Bantock's comment in The Artful Dodger and The Urgent Second Class about the ephemera, collage, photos and engravings points to the combinatory aspect of his work, which can be better understood in the context of the 
hypertext and web-fiction, and can be closely related to the way a work like Chemical Landscapes Digital Tales generates its narrative. Bantock's love for ephemera and the extensive use which he has made of them in his collages can be interpreted as his way of saying that the materials from which an artwork is made may have no intrinsic value by themselves and it is the combination of these elements that matters. This claim also mirrors Gass's ideas about the words which were discussed in the first chapter (The chair metaphor). This claim can be interpreted within the area of the materiality of the medium as well. Accordingly it is possible to say that there is nothing inherently good or bad or valuable or invaluable about the medium, whether it is a computer or a typewriter or even a pencil, rather it is the way that the medium has been used to combine these features that differentiates them from traditional print works. An artist/writer creates those combinations according to his own aesthetic taste, and in doing that brings together relatively unrelated material components, and changes their arrangement until he feels that he has successfully created a beautiful chair out of "smoothed sticks, or cloth or metal tubes." Here, for Bantock the sticks or tubes are the cheap and useless ephemera which he has found here and there and employs them to construct a work of art which is the chair in Gass's metaphor. For Bantock, the relationship of words and images to each other is so integral that it is impossible to think of one without the other, and this is the reason that all his books showcase this marriage of words and images.

\section{Physical Organization and Design}

Before my memories could fade, I set about confirming the solidity of the arc, and recording the events. Later when my account grew pictures, I decided to bind the words and images together in book form. The following is a limited edition of one. (Nick Bantock, The Forgetting Room n.pag.)

The phrase "a limited edition" at the very beginning of the book prepares the reader for the unique book that he is about to read. This book exists between the unique art work/paining, and the multiple-copy print text in which both word and image are presumably reproduced by digital/photographic methods. Apart from its linguistic component, this novel uses a variety of non-linguistic components such as a 
triptych, a concertina page, a wedding invitation card, and images which range from old maps and children's paintings to rich abstract pictures and collages.

From the margin of this book to its font, line spacing, the large space allocated to the image, and the pasting in of the concertina page, wedding invitation card and the final triptych, all indicate the high-cost production of this book which distinguishes it from twentieth century mass-produced books. According to the copyright page of this book, it is published by Harper Collins, designed by Isabelle Swiderski under the art direction of Barbara Hodgson. The jacket art is done by Nick Bantock, and features a blown-up part of the cover photo which is a collage of various ephemera such as a magnifier, a domino, knitting, the head of a bee sitting on a ticket; the photograph of this collage has been taken by Robert Keziere. The writer's involvement in the graphic dimension of this book ends here, and although he has created all the artwork for the novel, their arrangement and design has been the result of the collaboration between various artists, designers, and photographers who have been named on the copyright page. The distorted photo of Bantock's artwork on the jacket leaves out almost half of the elements which are seen on the actual cover of the novel, such as the domino and the golden crocodile paper clip which dominate the cover. What this comparison highlights is that by changing the ratios for the frame through which an object is seen, the object, or at least the appearance of the object, totally changes. In other words, the change occurs not in the object itself but in perception of it. An artwork after all generates a representation of an object within a certain frame, and this frame plays an important role in this process, and as it is seen here, it does not have a neutral role.

Apart from the frame, another important difference between the photo of the collage on the jacket and the one on the cover is that the former has words superimposed on a page. The three groups of words: author's name, name of the novel, and the short blurb of "a fiction from the author of best-selling Griffin \& Sabine trilogy" appear alongside this collage, and make it foreground the graphic surface of this novel which is the result of the intentional placement of the linguistic and non-linguistic elements alongside each other. 
The graphic surface of this novel makes itself visible through the many photos, pastings, and symbols that appear on almost every page of the novel Among all these organizational features, two elements require to be specifically mentioned due to their important role in bringing the materiality of the book to the foreground. The first one is the concertina page which is not ordinarily seen in books, let alone novels. Concertina pages, which usually appear in packaging of the music CDs and DVDs, literally comes out of the book, and can go outside the covers. The six folded pages include superimposed photos of different kinds, with each one having one letter and a number at the top. The pages are in numbered sequence, like the pages of a novel, but the content of each page is not immediately comprehensible. The main significance for this is that the sequential arrangement may not necessarily be employed for the sake of developing a case or unfolding of a narrative in a linear fashion. The sequential movement forward and unfolding of a linear narrative are just conventions which work well together, but they are not the only possible way of arranging the material and the way the concertina page has been organized is one of those alternative ways of arrangement. This page, and the experience of opening and closing the concertina page, are among the few occasions in this novel where the materiality of the book has been used to perform an action which cannot be transferred in the same way to the electronic media.

The second element is the triptych on page 101, which is the finished work of art by the main character. Stylistically the triptych in its closed position is very similar to a book which needs to be opened by the reader/viewer, a feature that is non-transferable to the digital media. The triptych, on the other hand, is not located on the wooden panels which are described in the novel, and a representation of it is pasted here in the book. Furthermore, the triptych has been historically used for sacred art, and its three parts (mirroring the trinity) have been used here to represent something which is not even remotely religious, but creates a new context for it which can be interpreted as irreligious. As seen above Bantock is trying to marry the word and image together, whereas in Christianity the picture has always been serving the word, because according to the book of Genesis "In the beginning was the word, and the word was with God, and the word was God." The very fact that the Bible does not include any images is another testament to the preference of word over image in 
Christianity; the triptychs which have been created for the churches then have been used to illustrate the stories of the Bible. In other words, by using the term triptych, and the three-part structure, the context of sacred art is invoked (and hence its high value). However, by not employing the image space to convey religious imagery, the painting disavows an especially religious purpose or meaning, but still claims the value associated with sacred art.

\section{Narrative Strategy}

The narrative begins with Armon Hurt(ago)'s trying to recall the eight days that he has spent in Ronda, Spain, and shows how he finally re-embraces his cultural heritage and literally brings back "ago" to his life and his name. In the same way that the book intends to marry the word and the image, the protagonist here also recovers his identity and brings"ago" back to his family name "Hurt" and in this way reclaims what he has lost by the previous generation. The narrative strategy of this novel requires the reader to pay particular attention to both the images and the words at the same time. As Bantock has said:

If you took a standard novel and knocked out every second word - that's how much sense my books would make if you took out the images. They're not illustrative, they're not subservient to the word, they are very much part of the narrative. If you choose to ignore them you're only getting half the story. (Bantock, interview by Gavin J. Grant)

The forgetting room of the title is the name of the studio where Armon's grandfather, ${ }^{34}$ Rafael works and this is the place that Armon remembers and rediscovers his "true heritage" which is learning to paint. Armon's efforts to regain this heritage results in his achieving maturity, which is depicted through his encounter with Ceres, a woman whom "a great scar [has] masked nearly half her face." When Armon sees her for the first time at the restaurant in Hotel Polo's, he is disillusioned by her physical deformity and fails to appreciate the beauty behind that. "My preconceived view of perfection couldn't accommodate her, and while I stared again

\footnotetext{
${ }^{34}$ And happens to be the name of Nick Bantock's own studio in Vancouver.
} 
at the beauty of that face struck by lightning, my tightly held sense of order and composition crumpled in on itself'(44) (italics mine).

What the narrator refers to as the "sense of order and composition" can be interpreted as the classic idea of beauty and aesthetics. In other words, it has been created by the conventions which through the centuries have been formed and developed. Whatever does not conform to these traditions might be thought of as ugly or a failure (when it comes to an artwork), whereas these conventions have limited the conception of beauty, and as the narrator observes, that "tightly held sense of order and composition" has blinded him toward her beauty.

By adding this character to the narrative, what Bantock is doing is providing a theoretical background for the way his novel and the narrative which it contains stands out among the works of conventional novels. Though his novels have not been particularly aligned with experimental novelists - there is only one reference to him in the whole volume of The Routledge Companion to Experimental Literature, where Gibbons refers to his Griffin and Sabine as an example of the epistolary multimodal novel-his works can be best understood in the context of metatextual novels, and it surprises him that his works have not found footing with critics and scholars. ${ }^{35}$

The introduction of this character is in a way trying to provide a rationale for the way the conventions of print as applied to the novel have normalized only one kind of arrangement of the words on the paper and they have not taken other works which play with their graphic surface and bring it to the foreground into account. The beautiful woman in this novel can be associated with the beautiful woman that Gass wrote about in his metaphor, but Bantock's insistence on her unconventional beauty in the context of his poorly-received novel by the academia, highlights how important Gibbons' observation at the beginning of this thesis has been. What makes the beauty of Bantock's novel particularly unconventional is that he has been treating the pages of his books as display surfaces and by doing that he alters the traditional arrangement of words on the page. These alterations can be in the form of adding small graphic

\footnotetext{
35 "Because my work incorporates art and lit it doesn't get covered by either camp. At first that bugged me, but now I've become philosophical and have taken the stance that operating between the tracks has way more advantages than disadvantages." (personal correspondence with the writer).
} 
and decorative sketches at the end of the last paragraph for each day of the narrative (because the narrative is divided according to days, not the conventional chapters), or they can be intricately designed and created artifacts that have been solely created for this book and have been added to it (the concertina page, and the triptych).

Though digital media are not specifically discussed in Bantock's novel, adding another artwork on page 27 labeled as "Instruction for the Forgetting Room" makes the experience of reading, more similar to the experience of reading a work of hypertext or web-fiction. The accompanying photo shows a "palascope" which has a typewriter at the bottom and a weird equivalent of what is supposed to be the screen (because the main character is supposed to look into that to see the verification form). TFR is after all a room in the big house that Armon has inherited from his grandparents, but the fact that this room, which shares the same title of the novel, comes with a set of instruction shows that this novel is quite different from the traditional novels, in the same way that TFR is different from the other rooms in the house. The instructions that a reader should follow will be discussed in the relevant section, but let us have a look at the set of instruction for the forgetting room, that Armon's grandfather, Rafael, has left for him.

- Look into the palascope and you will see the verification form.

- The button that illuminates the questionnaire also activates the voice control.

- Please type your name.

- Nothing will happen unless you type in a name.

- Incorrect statements will be erased.

- Please tell the truth.

- Try again.

- If the name you are using has been reassigned, the reclassification can only take place when due process has been completed. Please type in standard code-minus 123456.

- Once entered, proceed with the questionnaire until liberation is achieved. (27) 
As can be seen, in one part of the instructions, the person reading these should type in a name. Neither the reader, nor Armon need to type anything to unlock the mystery behind the concertina page (which these instruction help to resolve); it appears that the instructions are more suitable to a digital device that has a variety of features such as verification form, and voice control.

It does not take long for the reader to realize that the questionnaire that has been referred to in this instruction sheet is nothing other than the concertina page which entails the questions (almost all of them have only 2 words), and when the standard codes minus 123456 are typed, the name which emerges is DUENDE which becomes the key to resolving the mystery of the concertina page. Therefore, the concertina page becomes a map for the exploration of a greater mystery which exists in TFR, and resolving that would lead to liberation in both literal and figurative sense. Although the concertina page does not have any of those features which the instruction sheet has listed, the fact that concertina page includes an interactive engagement from the reader so that it yields what it has hidden through codes, in addition to its moving out of the book, points toward the digital media.

The narrative of this novel is created through the Armon's engagement with both the pictorial and linguistic elements, and it is through this engagement that he achieves the epiphany at the end. In other words both the pictorial and verbal elements help to give Armon a better understanding in his journey of identity and self-discovery. The narrative discourse of this novel follows the temporal progression; it starts from day one and finishes at day seven. The non-linguistic elements, on the other hand, complement the narrative in the sense that they show the step-by-step maturation of the protagonist through the stage-by-stage development of the collage in the end.

Some of the objects represented in the linguistic section of the novel are presented as found objects and are pasted onto the pages of the novel. The linguistic representation and their presentation through their existence as real life objects on the pages of the novel, offers a comparison and contrast of what each mode does and how they can both contribute to the liberated sense that Bantock has been talking about. The linguistic section carries the narrative load, but due to the abstract nature of the 
representation that it provides, it cannot replace the concrete representation that a picture provides. On the other hand, the images in the novel do not provide a narrative by themselves. But when word and image are married together, they offer a narrative which includes important themes such as identity, love, and maturation.

\section{Reading Process}

Originally I trained as a painter. Then I had to earn a living, and I did book covers and that sort of thing for a long time...Collage was a natural extension. All that was, was taking all those different techniques and bringing them together. In any collage you'll probably see anything between 10 and 15 different media mixed together. (Bantock, Interview by Gavin J. Grant)

The 10 and 15 different media which Bantock talks about are actually modes in the sense that though they both share many common features, as Gibbons states in her book "multimodality is a broader phenomenon, related to the coexistence of semiotic modes, whereas multimediality is created through the combination of media technologies" (Gibbons 19). Therefore, many of the media which Bantock talks about and then names, are in fact modes because they cannot be associated with media technologies, but, they can be used as semiotic modes. Particularly in this novel, these modes are the different ephemera that have already been discussed in other parts of this case study; however, what is significant about these modes is the fact that each of them used in a collage, including the one in The Forgetting Room, does not try to efface its materiality or that of the other media which accompany it in the final collage, but they all reinforce each other and add to the overall complexity and inherent harmony/beauty of the collage which has been created by the artist.

Bantock has taken experimentation with the images one step further and has included the mode of narrative writing - which has been mostly independent of images in its representation during the history of the novel-in his collage works. By claiming his books to be novels Bantock is asking the reader to treat his books as works of fiction; however, at the same time he wants the reader to expand her definition of reading by including the pictures as part of the reading experience. In order to achieve this aim he has foregrounded the graphic surface of his work which 
includes, but is not limited to, adding pictures and pasting in some artworks which disrupts the usual act of turning the page by the reader. On the other hand, since the central mystery of the novel is placed on the concertina page, the reader has to go back each time that the main character finds a new clue and have a closer look at the page that is being referred to in the narrative.

The reader does not have to perform the tasks which the instruction sheet presents, but the arrangement of images along with words in the novel makes her go through the non-linguistic elements of the text to see how it is possible to resolve the mystery at the heart of the novel. Here the image layer and the linguistic layer find a dynamic relationship because they are integrated together through the book as well as the mystery which the main character wants to resolve. This layering is further emphasized through the step-by-step transformation of Armon's simple sketch into the triptych. At each stage of development, a new layer is added to the artwork that he is creating, this new layer does not erase the layer below it, but adds to its complexity by enhancing what already exists there. The final artwork as it is seen in the last pages of the novel is the result of all these layers and none of them dominates it. This final artwork can stand for the novel in the sense Bantock has already wanted it to be, and already exists in web-fiction; a multimodal artwork in which different layers of mode co-exist and which is not transferable to other media. 


\section{Case Study 4.2. The Fifty Year Sword}

\section{Introduction}

The Fifty Year Sword is a metatextual novel by Mark Z. Danielewski published by a Dutch publisher, De Bezige Bij. Danielewski started his career as an experimental novelist and he continues his experimentation with the print medium in this novel by playing "with layout, color, and the wide potentials of perspective" in presenting its story. This novel tells the story of Halloween night in East Texas by means of fragmentary extracts from five interviews given in the form of direct speech. The direct speech related to each character is separated from the rest through colored quotation marks. A few years after the limited release of this novel in the Dutch publication, Danielewski conducted two live performances of this work in 2010 and one in each of 2011 and 2012 in which five voices (four of them female and one male) read their shares of the narrative, along with a large-scale (forty-foot) shadow-play by shadow-caster Christine Marie. After the American release of the novel in 2012, Danielewski conducted live performances in other cities and in one of them he read the whole novel to the audience. The new edition is shorter, and has lots of images created through stitching. Moreover an enhanced e-book version of this novel for iOS devices was released the same year (2012) which according to the iTunes website "features an animated cover design, animated illustrations and text, sound effects, and original music." Under the section of "A Note to the Reader" in the enhanced e-book version, the reader is informed that the animations, music and sound effects are activated by turning the page, and reactivated by returning to the page. In this case study, the original 2005 edition of this novel has been analyzed.

The media-consciousness in this novel has created an uncertainty, a place between borders, and a no man's land which is neither here nor there. The note on page six ends with: "so as to present here a pretty peculiar and perhaps altogether alternate history of one October evening in East Texas" (6). In White's interpretation of this sentence, this uncertainty has been hinted at: "the material... produces either something doubtful ('pretty peculiar') or simply false ('altogether alternate')" (Mark Z. Danielewski 107). This in-between and uncertain status is also followed in the 
narrative as well. The first word of the narrative is "Maybe" which emphasizes this lack of conviction. The reader is also offered two ways of reading, a) reading alone and b) reading aloud. Neither of these has been preferred. The writer as it was said above has performed it and read it aloud, conducted it for a group of artists, yet has highlighted the graphic surface of his book in a way which reading alone at times seems to be a better way of approaching the novel's graphic surface.

\section{Physical Organization and Design}

"It's a tiny story in some ways, but...the typography, the layout, the illustrative elements felt very much like they were realizing the [linguistic] text, like the text was incomplete without those elements" (Danielewski, interview by Elina Shatkin).

The Fifty Year Sword comes in a jacket, which along with its unique graphic surface has been designed by Danielewski. This graphic surface novel includes words, which only appear on the verso pages, and blue-ink illustrations which occasionally appear on recto pages and in some instances cover the whole spread. All of these illustrations - which have an abstract feel to them-have been done by the Dutch artist Peter Van Sambeek and will be studied in the next section.

An interesting point of comparison between this novel and The Forgetting Room is the way both writers of these novels have approached the image-word relationship in their work. The pictures here, in contrast to Bantock's novel, are obviously illustrations for the linguistic part of the novel. Despite this difference, Danielewski in his own way wants to achieve what Bantock had been trying to accomplish as well. This is what Danielewski has to say about word-image relationship in several interviews:

And there is something curious which I've found as I become more and more focused on this, which is when you move to a literal image, it does tend to change your thinking. It shifts you out of that verbal experience and it becomes something else. So, I think, for me, the exploration right now is finding that fine-line between text and image where it doesn't kick over into the image side of your mind. It actually still remains in the verbal, but it accesses the image without actually 
abandoning the verbal. That's the kind of tightrope that I attempt to walk and that's the challenge. (Danielewski, interview by Katherine Manderfield)

It's looking more closely at how image and text relate. It's walking this barbed wire with bare feet between two much image and too much text. There's really no such thing as "too much image" or "too much text," but there is a liminal space between both where imagination lights up in a slightly different way.... Finishing Fifty Year Sword was really about creating images that were coherent and intrinsic to what was textually stated and yet didn't go so far as to deprive anyone of an imaginative leap. The heroine is a seamstress, so it was important to use thread and to create blank pages that give your imagination a certain moment to pause and consider. It liberated me in terms of language and voice. (Danielewski, interview by Elina Shatkin)

By creating multimodal works, both of these writers aim to provide a rationale for their use of the images, but their primary concern in doing that is that neither the words nor the images take over the text. The main result of highlighting the graphic surface is that it makes the text a visual space. Both Hayles and Gibbons had highlighted this visuality in discussing the digital-era experimental novels too. In addition to playing with the arrangement of the words on the pages, adding images/illustrations to the linguistic section of the novel brings another mode to the novel and meanwhile intensifies this visuality. But the inclusion of images also requires maintaining a balance between these two modes and each of these writers in his own way has been trying to create this balance. Bantock wants to marry them and thus avoid becoming the illustration/annotation of the other, whereas Danielewski wants to keep them apart and looks forward to walking the tightrope/border which separates the two. Therefore, as can be seen, both of them seek to achieve the same effect but they follow different paths.

Although images make a significant contribution to a metatextual novel, the metatextuality of The Fifty Year Sword is the result of the way Danielewski wants to highlight the graphic surface of his work in various ways. This "disruptive" graphic 
surface, as White in his short essay on this novel discusses, creates a work which in many respects reflects Danielewski's perception of the borders and can be best described as a novel which is in-between. The other unique features of this novel include: its unusual size - which its similarity to a roll of fabric or a sword box, among other things, will be explored later-its fonts, and the colored quotation marks. Each of these will be analyzed in more detail in this section.

As it was said earlier, the in-between status of the novel dominates this work. It is a novel, but the arrangement of the words on the paper makes it more similar to poetry; the illustrations suggest scenes from the novel but they are not as distinct as illustrations traditionally should be; the story is described as a ghost story, yet there are no ghosts in the story; the Story Teller narrates a story for children which is totally different from what one may expect from a traditional children's story, etc. Although this in-between (or border) status of The Fifty Year Sword makes it harder to describe, by remediating other media Danielewski places his novel on a border between the traditional novel as a special case of the medium and the book as a metatextual object.

The layers which were discussed above here appear as threads and, according to White, Danielewski's novel "weaves its five threads of narration into a pattern, to deliver a climax as cryptic as the origins of the Story Teller's hate" (Mark Z. Danielewski 119) What his pattern is and its "cryptic" qualities will be analyzed in the next section, but what is important here is the way these threads/layers have a dynamic relationship which in many ways mirrors the textuality of the cybertexts. In The Fifty Year Sword there is no specific order for the way each thread/layer appears. These colored threads/layers appear in various horizontal and vertical orders which "disrupts" any specific order/sequence that might have been expected from them.

\section{Unusual Size}

The Fifty Year Sword has been published in a long and narrow format (121/2 $\mathrm{x}$ $61 / 2$ inches), its height is a little longer than height of quarto format (12 x $9 \frac{1 / 2}{2}$ inches) and its width is a little more than the width of the octavo format ( 9 × 6 inches). This in-between-ness places the book on a border between these two formats and while it retains aspects of each one, it does not belong to either. Two of the possible 
interpretations for this unusual size of the book can be a sword box and a roll of fabric, each of which will be studied in this section.

Since the words text and texture share the same Latin origin which has been discussed in Chapter Two, it is not surprising to see that both Joyce and Danielewski have used this etymological feature in their own different ways to remind the readers of this similarity. By cutting the interviews into hundreds of segments and using around 2000 of them in his text, Danielewski has created a novel whose parts need to be stitched together by the reader. On the other hand, since ultimately he has been in charge of adding/removing these bits to the print book, he claims that the final result might be an alternate version of what has happened that night in October. By using the phrase "alternate version" he is emphasizing the non-representational status of his work and indicating that the sum of all these threads is not a clear and explicit account of the events of that Halloween night. Having this information in mind, it is possible to think of different patterns to emerge from the fabric of the book. Though the book has a clear pre-organized sequence for the threads to appear in it, the possibility of the absence of a real or one-to-one correlation between the events narrated in the novel and the events of Halloween night, encourages different kinds of reading, two of which, reading aloud and reading alone, will be analyzed in the last section of this case study.

Looking at the novel from this angle makes it easier to understand why sewing/stitching terminology and metaphors abound in this work. The grainy and almost pointillist quality of the cover image makes it more similar to an image which has been either printed on a piece of fabric, or an image which has been created by stitching several threads in a piece of fabric. In this case, the long and narrow size of the book makes it similar to a piece of fabric which has been woven together from those five threads by M.D. ${ }^{36}$ This comparison is supported by the focalization of the narrative through Chintana, who is a seamstress and who uses her knowledge of her work and textile terminology as a way of thinking about how people relate to one another and meaning is created through narratives of events.

\footnotetext{
36 The introduction has been signed by M.D. which may or may not stand for Mark Danielewski, because there is at least one other M.D., Mose Dettledown, in the narrative. She is the woman who throws the party and invites Chintana.
} 
The book also can stand for a sword box and this similarity is encouraged by the way the limited edition of the book published in 2012 is placed in a box with five latches. In both editions, the words in the note on page six of the novel have been arranged in way which is similar to the hilt of a sword, and from this angle the rest of the novel can be the blade of the sword which can be either visible as the words arranged in long and narrow columns on the page are, or invisible as the white pages are and the blade in the sword of the Story Teller is, but which affects the reader/audience after he has finished reading the novel. There are also several references to swords, cutting, and killing in this novel. It is also the Story Teller who brings in a sword box to the Halloween party and uses it as a frame/reason for the words he has been narrating. The swords on the cover illustration, as they are described later in the narrative, are no ordinary swords either, and their odd effects on their victims (killing tastes, colors, etc.) are not what is ordinarily expected to be the result of a sword wound.

\section{Font}

As soon as I start writing, I am already dealing with the visual aspects, and constantly examining and re-examining what font I am using, what kind of textual layout I am exploring, how those textual layouts are gonna intersect with other layouts, graphic elements, what is too much graphic, what is too dense verbally. (Danielewski, Interview by Carolyn Kellogg)

The above quotation also makes it clear what the writing process is like, and how the space of the page and the massing of type elements interact in the instantiation of his conception of the work. It also shows that the conception is not whole in his mind to be delivered to a medium, but the process of realizing the conception is interactive between the medium and the idea. This makes the writer a guide who seeks to involve the reader in this interaction and, as we will see later, he achieves this by bringing in the different fonts and stating their names on the copyright page of the novel in order of their appearance. ${ }^{37}$ Arguably the fonts have

\footnotetext{
37 He later goes one step further for the American edition and states the industrial names of the colors used for quotations
} 
always been used as visual markers, asserting or supporting values like elegance, scholarship, force, speed, power and so forth. Stating the names of the fonts in this novel opens up another interpretive layer which will be discussed in detail in relation to the reading process. The words of the note on page six which is the only page of the novel on which the words appear without quotation marks have been typeset in Dante font. Here, this font separates the note on page six by M.D. from the rest of the novel which is narrated by the five narrators who have been described in the note on page six and use another font, Legacy, in their narration. The Legacy font shows the common heritage of this fateful night on the narrators whom the use of common font for them affirms what is common to each; the legacy of that night which is shared memories. The words "Dante" and "Legacy" on their own and coupled together also create a wealth of possible interpretations which can help reinforce the in-between status of the novel. Since Dante, whom as we see later is the reader's guide, does not move beyond the threshold of the novel, the rest of the novel can be thought of as the purgatory which is neither heaven nor hell, but a world in between.

\section{The Colored Quotation Marks}

Another distinctive feature of the graphic surface of this novel is that it uses colored quotation marks for the different narrators of the novel. The first part of the book on which the colors appear is the jacket of the novel where the five parts of the title along with the writer's name at the top are arranged vertically. The colors which have been used for each word are the same ones which have been used for the narrators within the novel.

White describes these colors as 1) deep yellow, 2) orange, 3) red-brown, 4) mid-brown and 5) dark brown. What stands out in White's categorization is that except orange, the rest of the colors are the ones which are in-between other colors and White has had to use adjectives such as deep, mid, and dark to distinguish them from each other. These five colors, as Danielewski states in his interview by Kasey Carpenter, stand for the colors of autumn and the spices. This yellow-to-red range of colors for the autumn and the spices, stands in contrast to the snowy whiteness of the blank page and the shadowy feel of the whole novel. However, the similarity between them and the way they are printed in the book make it hard to distinguish among and 
this the reason that White aptly says, "The colors of the five quotation marks are related closely enough so that the darker ones often require comparison between one another to be definitely attributed" (108). This close range of colors for the quotation marks makes it harder and harder to follow who says what and the more the narrative moves forward, these quotation marks play more tricks and in various part of the novel, several of them come together.

\section{Images}

The images in this novel support the in-between status of the novel too. They vary quite a lot from the ordinary illustrations for a traditional novel in which the illustrations typically offer vivid details of the characters created from the novel's narrative either with or without the cooperation of the author. Altogether, there are five full spread images and seven single-page images. All of them are in blue-ink color and have a grainy look which, as was discussed above, makes them look as if they have either been printed on a fabric or created by threads in the fabric. These images in their present form suggest a scene or part of a scene, but do not fully illustrate the narrative which accompanies them. Part of this semi-illustration is that they reflect the uncertain feel of the linguistic section. At times they linger between representation and abstraction. These images usually fill in the blank spaces of the novel and in this way, apart from the five spreads, they do not affect the linguistic section of this book.

\section{Narrative Strategy}

The in-between-ness identified above appears at the level of the narrative too, it is a narrative about a seamstress whose heart has been cut, there is a tendency in the narrative to cut, take, apart, analyze, separate the threads, which is complemented by the opposing sewing metaphor, also the narrative binds fragments of memory and perception/perspective into a story which is something more than the sum of the parts, etc. It is not surprising then that the butterfly is the perfect motif for The Fifty Year Sword, because its opposing meanings fit quite with the overall status of this book which is neither this nor that. 
Another support for the in-between status of the novel in its narrative is the media consciousness which is evident from the unique way it takes advantage of the affordances of the medium. This novel aims to remediate the campfire story through the book, but instead of having a character like Joseph Conrad's Marlow who narrates the story from the start to finish, he has five characters whose narratives have to be put together to form a possible narrative. The in-between status of the novel helps to clarify the novel's uneasy placement as a book; it tends to be read aloud and meanwhile it wants to be read alone. It is both a novel, and a play, yet it doesn't form allegiance with either. It is hard to categorize The Fifty Year Sword under any of the existing genres of fiction, because it occupies borders of different genres, without declaring allegiance with any single one of them. It is marketed as a "ghost story for grown-up readers" by its publisher, but the typical ghost story elements are absent from it and the only ghosts which appear in it are the ghosts of the Story Teller on his way to find "the man with no (h)arms," and those ghosts have such a minor role in the story that they do not help to qualify this story as a ghost story. The jacket of the book claims that if "read aloud, The Fifty Year Sword will captivate any child," but this novel sits uneasily in this category of Children's Literature too, because this dark story of betrayal, divorce, vengeance and raw violence would hardly succeed in convincing any parent to read it to her children. Even the story that the Story Teller narrates to the five children is so different from what Chintana and other listeners expect that at some point Chintana thinks about stopping the Story Teller and shifts "uncomfortably./ Where were the animals,/ the gleefully abandoned, or/ the stormy skies/ appropriate for a/ ghost story for the young?/ Where was the comedy"(43)? ${ }^{38}$ That story is too dark and grim for the children which, according to the Story Teller, they will not understand until they grow up and it becomes part of their "legacy."

This in-between status of the novel becomes more complicated when one looks at the way the Library of Congress in its CIP (Cataloging in Publication) record has categorized it as 1.Children-Fiction, 2.Revenge-Fiction, and 3.Texas, EastFiction for the 2012 edition. According to the website of LOC, CIP data which is a bibliographic record is "prepared by the Library of Congress for a book that has not

\footnotetext{
${ }^{38}$ In order to avoid confusion, the original colored quotation marks have been omitted from the quotations cited here.
} 
yet been published" Apart from children's literature, two other categories (revenge, and East Texas) have been identified by the reviewer of LOC, and it is possible to justify these two subcategories by finding related material in the novel. This range of different classifications supports the claim that the in-between status of the novel has made it difficult to fit in any specific category.

The theme of "revenge" is something that one can find more evidence for within the novel than the other categories. This theme is present both in the story of Chintana and the one narrated by the Story Teller. From the beginning Chintana is so furious with Belinda Kite that even Belinda who has "the sharpest tongue in East Texas" realizes that "one word, even the slightest/ indacitation of intimacy could very well/ have brought about her own/ extinction." Chintana is so desperate for revenge that "despite pacific beliefs, only infliction/ promised her peace" (8). Although he does not specifically state it, Story Teller is also seeking revenge. The narrative does not offer any glimpses of his life before he starts his quest for the deadliest weapon. $\mathrm{He}$ is not certain what has made him to go on this quest either and he only says:

But also because you are/ young, I will not tell you why I went/ in search of such a weapon, though/ in truth while I could speculate/ I am no longer capable of/ recalling the details myself./ When you are older/ you will be able to imagine/ what drove me on such a/ quest./ You will know then more than me. (40-42)

So although one can never be sure about what the Story Teller's quest has been, it is possible to infer that he has wanted that weapon for revenge. However, in that story the man only vaguely remembers the reason for his quest and the revenge (or maybe it is the price that he pays for the sword), and he does not use the sword to kill anyone after he acquires it.

The East Texas Fiction category is more a reference to the setting than being a theme in the story; apart from its mention in the note on page six, it is highlighted by bringing in the East Texan dialect into the novel in two ways. The first one is using alternate spellings for the words such as "consecwantly," or "chear", which when read aloud have the same pronunciation as the correct written form of the word. The second one is cutting some letters off the words or stitching their letters together in a 
different ways (Suprising, probely) which are still recognizable to the eye and ear and in some cases adding new letters to them (acceptate, reconsiderated, indacitation). This change in the linguistic format of the words emphasizes the in-between status at the micro-level of words too. Here the words carry the semantic load which their "correct" linguistic form does, yet they are not the in the correct form, and their incorrect spelling provides the opportunity for suggesting different meanings as well. So as can be seen the narrative of this novel, despite its similarities to the other genres such as children fiction or horror fiction, defies classification and retains an in-between-the-genres status, and meanwhile preserves its claim to being a novel.

This status is mirrored again in the presentation of the narrative in the book format too. The whole narrative is focalized through the eyes of Chintana, but almost all segments of the narration are in the usual third person, so when Chintana is referred to, for example, it has to be by a speaker who is not her. But it is the author/narrator who has chosen the segments of speech from each interview to construct this version. As we can see in the same note on page six, there is very little information about the sources of the story, even the information that MD provides about their background offers very few hints about their love and hate relationships. What can be inferred from this information on the handle of the story is that the relationship of these five sources to the narrative is a flat relationship and none of them is preferred or made more significant; they interrupt, quote and finish what each other and the narrative is created through the complex layering which these narrators make possible. Meanwhile the personal history/memory affects their narration too and this can be one of the reasons for the visible discrepancies in their stories, for example, Chintana's name is changed to Chantana in the last few pages of the novel, and Dettledown becomes Dettlesome.

\section{Blank Spaces}

The empty space of the page would traditionally have an illustration, that is, a translation of the referential/descriptive dimension of the words into an image which is to be taken as standing for the presence of the (real) world which is the setting of the narrative, the visible part, but the empty spaces in this novel (as we saw in the print version of 10:01) are intended to signify the void which stories attempt to fill. 
The story we have by means of the fragments from the five narratives/interviews does not refer to or stand in for the true (that is, the unique account and hence not a version) story - it is only ever possible to write versions.

Danielewski at various points emphasizes this "readerly" approach to his text and openly admits that the blank pages are the spaces of co-authoring the text for the reader. But what is relevant for the way that physical organization and design ties up with the narrative here is how the whiteness of these blank pages can be related to the snow which has covered the land in the last few pages of the book:

No, one else would have/admitted the possibility/that someone else had/seen so, or/saw so,/caught a glimpse/now and then of something/slippery,/ biting through the air,/ white and vague,/ like a slow cold fog/creeping across/an evening after/ a burial.(88)

Belinda Kite/because she claimed to adore/the snow had demanded her/toast take place in it. (90)

the children/were the first to see the snow/splash/red.

In the same way that the snow has covered the land the whiteness of the blank space has covered almost four fifths of each spread. Since the text is printed on the left hand side of the page the author would have wanted the eye to go over the white space before moving into the next line. In another interview Danielewski directly comments on the blank spaces and their effects on the chunks of text:

Now: just imagine, if all of the text had been on the right side, and the left side had been blank, it would've been much more closed off. And your eye would've just ignored the blank page and gone on to the next chunk of text. It never would've pondered the blankness, the invisibleness, like the invisibility of the blade of the sword. (Danielewski, interview by Stacey Knecht) 
As can be seen from this quotation, the choice of having the blank spaces on the right side of the text both within the same page and the next page, creates momentary pauses within the narrative which highlights the existence of the white space and the narrative which has been silenced, or cut out, of that part.

\section{Butterfly}

The butterfly is the perfect term/image for this novel because it shows the in-between-ness and uncertainty through both its physical shape and its opposing meanings in the English language. This word which appears both as noun and verb in the novel has several implications here. The first one is the butterfly effect which in simple terms means a large output from a small input. Here in the story Belinda Kite is torn to pieces for "a simple act of mercy," that is sleeping with Pravat, Chintana's husband. Although it has not been a serious issue for Belinda, this affair has ruined the relationship of Chintana and Pravat. Even the final outcome of this affair for Belinda is huge too, and if we consider Belinda's dismemberment by the sword as the "infliction" which "despite pacific beliefs...promised her peace" we also see that a small input, i.e. sleeping with Pravat, has a huge effect which is not deserved. Here the meaning of butterfly as a verb supports this claim too. As a verb, butterfly means splitting almost entirely, so we see Belinda is being butterflied at the end of the story. On the other hand, butterfly is a kind of knot and surgical tape which the surgeon tapes on Chintana's finger and stitches the wound back after she cuts herself with scissors. Here the butterfly is a symbol of joining together rather than cutting up. So we see that butterfly has a double significance of both cutting up and stitching together. This double significance can be traced to the movement of the butterfly too. Here the text has already been cut up by the writer by adding in the quotation marks and the white space, the reader is expected to construct a coherent narrative which line by line, phrase by phrase nearly always opens onto a blank page, which in other words is a process of constantly stitching up and cutting, cutting a phrase from what is on the page and stitching it to what he has already read.

\section{Reading Process}

I think the question in The Fifty Year Sword is what is the real story, it is a fantastical story, it is characterized by a very strange sword, and by 
a very questionable violent ending, but at the same time, there is also the lingering possibility that what really happened that night is completely different, so you and I could be sitting for hours telling a story, but if we reassemble our words and our little snippets of our speech in a different manner we could potentially create a different story. (Danielewski, interview by Carolyn Kellogg)

The two modes of reading produce different results and according to the description on the jacket written by the jacket designer, Danielewski: "Read aloud The Fifty Year Sword will captivate any child. Read alone the complexities of nameless voices, misplaced narratives of hate, and the horrors of delayed violence, which only our retellings can rescue and restore, will thrill and touch the sharpest reader." What follows is the exploration of how each reading works and how each one achieves the aim which has been described above as a possible result of the reading.

\section{Reading Alone}

I feel one guiding covenant with the reader is to provide that which is unavailable anywhere else. It's not a question of whether it's liked or despised but whether it's unique. (Danielewski, Live Q\&A on io9.com)

When read alone, The Fifty Year Sword preserves its in-between status which has been discussed in the physical organization and design. The book is described as a novel, yet the arrangement of the words on the page are more similar to poetry. The poetic quality of the language has prompted some reviewers to call it a prose poem which seems a more accurate description because it refers to a genre which is in itself in-between prose and poetry. In almost all novels, even the experimental ones such as House of Leaves, the dialogs are mingled with narrations, but here there is no narration in this novel and the linguistic part of the novel merely relies on the dialogs. This feature makes it more similar to a work of drama. But despite its being a drama in which the cast of characters are identified by name, and in which is possible to identify who is saying what, readers do not have the opportunity to locate the sources of the narrative because they are deliberately kept in the dark. 
These aspects make it more difficult to read the first few pages of this novel, and the reader might get distracted by trying to find who is exactly saying this or that line, but the similarity of colors and the disorderly fashion in which they appear on the page makes this reading a cumbersome and difficult one. So a more rewarding reading can be achieved by following the flow of the narrative as it unfolds through these voices. Thus, the reader has to stitch different colors together to be able to see how the meaning of the linguistic parts emerges. Meanwhile in this reading the fonts, colors, blank spaces, and the images play an important part. Some of these features have been already discussed in the previous section, but it is also informative to briefly discuss them from a reader's perspective.

An important feature which is highlighted in this novel when it is read alone is the way that the author has used two different fonts for this work. The first one, Dante, reminds the reader of the real Dante and his famous Divine Comedy (1555/1993) and can be linked to what Danielewski in one of his interviews calls leap of faith:

What has gone unchanged since that time of teenage scrawlings - taught to me, by Dante himself, who was taught by Virgil, for yes, so are we all taught who read-is an understanding that the author must first and foremost serve as a guide leading the reader to tread beyond the limits of her or his certainty, to generously and responsibly take each (because the reader is never a Them) to the threshold of the imagination and encourage a leap not to the stars or even beyond the stars but beyond the compass points of personal belief....In order to leap, we first need to practice leaping. Dante leads the reader through a series of levels, literally, where leaps of imagination or association are learned by way of Virgil. Eventually though, Virgil departs and the reader has only Dante. Then Dante departs and the reader must, by way of example and practice, finally take the action every good book should in good conscience prepare the reader for. In the case of Divine Comedy Dante leads us to take a leap of faith. (Danielewski, interview by Matthew Treon) 
In this novel by using the Dante font, which has a reader-friendly look, the writer of the note on page six, M.D., takes the reader's hand and guides her across the threshold of the novel, briefly introduces the five narrators, talks about his reasons for using the colored quotation marks instead of "characterizing phrases, temporal reference," etc., and finally acknowledges his own role in the representation of the story. Then M.D. departs and the reader is left alone "to tread beyond the limits of her or his certainty." What the reader faces after this point is typeset in Legacy font. Legacy's connotation, which is something of value from the past preserved for the future, is used for narrating an event/history from the past, something which has remained from a previous time, and is now be re-narrated and re-told. If we think of The Fifty Year Sword as the sword, it can also be said that this book by bringing in something from the past and passing its invisible blade through the readers makes them carry this legacy into the future.

Another feature which draws the reader's attention to itself during her reading alone is the colors. These colors which have been discussed before, in addition to the "fall and spices", also remind the readers of the colors of the fire, which can be linked to the campfire story which this novel is remediating. So there are all these words moving back and forth between the flames of fire which at the same time have gathered the narrators and their audience there.

Since the novel has taken away a great many of the certainties and securities of the reader, what the reader is left with is the medium itself and is obliged to create connections between the different modes presented here. One of problematic issues is the images and how they are related to the narrative. What follows is an effort to address this issue where every image has been accounted for and its relationship to the narrative is analyzed.

\section{Illustrations and Their Relation to the Words}

What is explored in this section is the images and their relation to the text, and what Danielewski intends by having indistinct illustrations in his work. The quality and placement of the images within the novel also contribute to the in-between status that was previously discussed. The images are referred to as "illustrations" but they are not illustrations in the traditional sense of the term. As the story does not offer 
literal images, the illustrations do not offer vivid pictorial equivalents for the characters/events/scenes suggested by the verbal narrative either.

The first illustration is a two-page spread of Chintana holding a piece of fabric on her lap (the same piece of fabric is repeated in the final spread where Chintana is holding Belinda Kite in her arms). She is wearing jeans and a T-Shirt and there is an orange-red thread on a long piece of fabric which is explained on the next page to be the drapes. Apart from the figure of Chintana and the drape, another element in those illustrations which has an emphatic presence is the long and narrow leaves of what appears to be an evergreen tree or shrub. The evergreen tree in this illustration is associated with Chintana because, despite all the hardships that she undergoes, she keeps her composure and her calm and stable personality is contrasted to Belinda Kite who changes her mood more easily and, as we will see in the last illustration, is associated with deciduous trees. Chintana's gaze is toward the left side of the frame which can be associated with the past, when she cut her thumb with a pair of scissors.

The second full spread image on pages 16 and 17 comes at a more strategic point of the narrative, because it creates a pause in the reading while the narrative is describing what would have happened to Belinda Kite if she had said something when she sees Chintana arrive at Mose's house on the Halloween night, "the sharpest tongue in East Texas knew/better. One word, even the slightest/ indacitation of intimacy could very well/have brought about her own" (14). The reader here has to go through three pages before the next word "extinction" appears on the top of page 18 . Here the words are pushing the narrative forward, but the still image suspends the motion of time and by doing that suspends the narrative flow. Since this image appears between the segments of narrative, it can be argued that it represents what is unrepresented by the verbal narrative, because it appears between the segments. This illustration is a drawing of a right hand which, as we will see in the last illustration, belongs to Belinda Kite, who is wearing jewelry and a watch which shows that the time is almost 11:00. To sustain the suspense and not reveal the identity of this person, the thumb does not appear in the image. In the background to this image, there is a blurry drawing of a tree with yellow threads at its bottom. Those yellow threads can be associated with the fallen leaves of the trees in the fall which is the setting of this novel. The significance of the hand in this illustration is that it shows the fingers 
before they come off in the segment of the narrative right before the final illustration, where the five orphans "saw all five/fingers slide away and/tumble to the ground/in a soundless spray of blood" (94). Looking at this illustration from this point of view, it will be possible to re-interpret the orange threads at the bottom of the same illustration as a foreshadowing of Belinda's blood being spread all over the yard. Another reference to this image also appears when Chintana in reference to her own injury says she "could lose a hand" but the Social Worker says she "could lose more than that", which again foreshadows what happens to Belinda in the end.

The third image appears on page 29 , which is the first single page image. It is the most abstract and indistinct of the image of the book; it is also the first image in which a butterfly appears. The indistinct quality of the image can signify the fast movement of the orphans which is described on the facing page as "five white-tailed deer in race,/ fleetly bounding by/ and around the knees of/minglers and new comer,/whippoorwills!/ Cedar waxwings on air" (28). The butterfly at the bottom of the illustration, which as it has been discussed before, can stand both for bringing together and cutting apart, and for the opposite emotions/feelings that Chintana is experiencing. On the next page, we read that her sister is asking her to have gratitude and gentleness, but Chintana finds it hard to practice: "But just breathing required/ so much force it was almost/inconceivable to imagine gratitude/for what or who?/What about Belinda Kite" (30)?

The next five images directly refer to the story which The Story Teller is narrating. The first one of these, and the fourth image of the book, refers to the part where the Story Teller is telling the orphans about the time that he was taking cover from the rain and overhears a man talking about the man with no arms. The fifth image is a full-spread of the Valley of Salt, the sixth one is a full spread illustration of Forest of Falling Notes, the seventh one a single page of the Mountain of Manyone, the eighth illustration is of the pond where the Story Teller wakes up at after he finishes climbing the mountain of manyone, and the last Story Teller-related illustration is the image of several hilts which also appears on the book cover. The next illustration seems to appear unexpectedly on the right side page, because it does not seem to have a direct relation to the words of the story. It appears on page seventy five and it is a sewing box with a scissor and lots of buttons, needles and spools; on 
the facing page the Story Teller is narrating how he paid the price for his fifty year sword. As discussed in the previous section, what the Man with No Arms takes out of his head is the memory of the revenge, and the scissors and the butterfly which is on one of the spools reminds the reader of the inner desire of Chintana for revenge and how it is at odds with her profession which is bringing together and stitching.

The penultimate illustration is of several watches, two of which show the midnight time, and one of them has been cut open and its pieces are all over the box. This image appears next to the page where Belinda is asking others to have a toast for her birthday; at the same time it shows the time and what is going to happen to Belinda now. It also complements the meaning of the sewing box image because, although time is a great healer, it also can be the symbol of mortality and in this sense a great destroyer.

The final illustration, which shows Chintana holding the injured Belinda, complements the very last sentences of the novel in which it is said that

Just as she held onto,/ Belinda Kite,/ gently and painfully and/ gratefully too,/ acceptating them all,/ and with great sloppy/breaths too, even if she also/continued to wonder/just how long one/such tiny stitch of,/well you know,/ could really hold (98-100).

Three deciduous trees are shown in the background, they all have lost their leaves, and since this kind of tree was associated with Belinda Kite, her fall here has found a pictorial equivalent in the trees. Her red blood stands out against the blue-ink background of the illustration, and though there is only one drop of blood on the recto page of the book where Chintana is sitting and holding Belinda Kite, the narrative suggests that Belinda's final demise would be similar to the trees and, since no one knows "how long such tiny stitch of,/well you know/could really hold", it is only a matter of time.

The complex relationship of illustrations to the narrative has been designed to fulfill the aim that Danielewski had wanted the illustrations to play in his work. Their details brings in elements which have been absent from the narrative, such as the trees 
or the clothes of Chintana and Belinda, and at the same time do not offer pictorial equivalents for the words.

\section{Reading Aloud:}

Reading aloud makes the listener(s) pay more attention to the sound of the words in addition to their meaning. This kind of reading also provides this opportunity that each specific color is read by a single person. On the other hand the reading alone makes this similarity of this novel to a drama more apparent, but as the narrative progresses forward the embedded quotes within quotes distort this similarity and once again place this novel in-between a work of drama and campfire story.

\section{Conclusion:}

"A sense of vulnerability hangs over the narrative of The Fifty Year Sword." (Glynn White, Mark Z. Danielewski)

This sense of vulnerability that White has felt can be accounted for by the effects that the media consciousness of this novel has had on its presentation. Bringing in the other modes to the novel has made Danielewski create a novel which moves the reader over a tightrope. The novel takes away the securities and makes the reader follow its metatextual structure. Danielewski, like Bantock, is unhappy with the current status of word/image relationships and aims to modify this relationship by choosing to walk the borderline between the two hemispheres by putting the words which do not offer literal visualizations along with the images which have blurry representations, and playing with the graphic surface of the novel in a variety of ways.

\footnotetext{
${ }^{39}$ It also becomes quite challenging for the reader/performers who are reading a single color aloud, and this is where Danielewski's role as the maestro becomes quite significant in the live performances that he has "orchestrated" in Red Cat.
} 


\section{Case Study 4.3. House of Leaves}

\section{Introduction}

Little solace comes

to those who grieve

when thoughts keep drifting

as walls keep shifting

and this great blue world of ours

seems a house of leaves

moments before the wind

(House of Leaves 563)

House of Leaves, Danielewski's debut work of fiction, is a metatextual novel which incorporates almost all of the theoretical issues discussed in this thesis. In this novel, Danielewski brings the materiality of the print medium to the foreground and shows how the dynamic relation between the physical resources and the signifying strategies plays a significant role in what the book is (re)presenting. This novel abandons representation in the sense that it totally invalidates the existence of a real world object and comes closer to Gass's definition of a work of art; not a window onto the real world but an object within that world "worthy of love."

Several scholars (such as Gibbons and Hayles) have written about this novel but what distinguishes the discussion in this case study from their analysis is that this novel is explored from the materiality perspective and though at times the discussions here cover the same issues which Hayles has discussed in Electronic Literature, her discussions is more limited to trace the digital birth of the novel in that book and the post-human subject. 
At the core of the House of Leaves, there is a movie about an impossible house, the inside of which is larger than the outside. Will Navidson moves into this house and it does not take long until they discover this architectural anomaly and invite others to explore it. This documentary, "The Navidson Record", is narrated and commented on by Zampano whose pseudo-scientific essay/thesis on it, The Navidson Record, is discovered by Johnny Truant who adds his own narrative to the book in the footnotes and hands in the manuscript "House of Leaves" to a publisher, where a group of editors add their own comments to Johnny's manuscript and publish it as House of Leaves.

Media consciousness in this novel makes its several mediations apparent, and is shown through several comments on the recording devices which are used in each narrative layer. Navidson and Halloway's equipment in the first layer have been described in detail in several parts of the novel by Zampano, whose inscriptions on a variety of surfaces and by various writing options have been, in turn, described by Johnny Truant.

"The Editors" just say that they have received a manuscript which hints at Johnny's organizing Zampano's notes into a publishable manuscript. There are several hidden/explicit codes within the novel, which a community of users has been trying to decode. Many of those explicit codes have been borrowed from other modes, which along with many other modes create a richly multimodal object that remediates film and (at least until now, late 2013) has resisted being repurposed into film. Danielewski's resistance to sell the film right of this novel highlights the print-specific affordances of this book which cannot be transferred to other media. Finally, there are several layers in this novel, both in its narrative, and its physical organization that have different relationships toward each other, but as we will see this layering moves out of the novel into the digital media as well.

As already observed, the wide range of remediations in House of Leaves led Hayles to apply the term "hypertext" to this print novel. Hayles is not alone in assigning digital media features to this novel. Martin Brick goes as far as saying that, "the plethora of footnotes, appendices and divergent narratives would make a hyperlinked version of House of Leaves convenient to read, eliminating the need to 
turn the book, to endlessly flip through pages and to use two bookmarks," ("Blueprint (s)") and later goes on to conclude that "one might contend that HTML more faithfully represents the structure of Navidson's house" (ibid.). Though print has proved to be an equally suitable medium for this novel, Martin gets closest in discovering the range of the effects that the digital medium has had on metatextual print fiction and how this novel, by maintaining its allegiance with print, wears its digital birth and its ongoing relationship with other media, especially digital media, on its sleeve.

House of Leaves foregrounds its graphic surface in various ways. Some, as discussed throughout this thesis, have been used consistently by other metatextual novels, but some of them have been not been used as frequently as the others, and the wide variety of graphic devices used in this novel make it an encyclopedia of graphic devices which a print novel can use. This does not mean that House of Leaves has exhausted the store of graphic devices; other writers such as Jonathan Safran Foer in his novels (Tree of Codes (2010), Extremely Loud and Incredibly Close (2005) have shown that there are still many ways to make use of the graphic surface of the novel. However, the wide range of these ways in Danielewski's novel makes it an extremely difficult task to go through all of them and analyze each one in detail. Therefore, what follows is a selection of those graphic devices which have been most related to the issues discussed in this thesis.

\section{Physical Organization and Design}

"The image of him reading the story that contains him presents us with a vivid warning that this book threatens always to break out of the cover that binds it." (Hayles, Writing Machines, 129)

House of Leaves comes in four different editions; full color, 2-color, Black \& White, and Incomplete, all of which are second editions, because the first edition of this novel according to the Foreword, had been "privately distributed" as a digital file uploaded on the internet. "The remastered full-color edition" used for this case study, was published in 2000 by Pantheon books. The phrase remastered full-color edition is usually used for movies which have been improved - remastered-by using digital technology. By bringing this phrase to the top of the cover, the book is hinting at its 
relationship to digital media, and at the same time revealing how this process of being remastered has helped to create an artifact which differs from the other editions (at least two of them) and has led to the creation of a different object, the materiality of which plays a vital role in the way it is interpreted.

In addition to the cover and the title page, the colors, fonts, color plates, footnotes and the arrangement of the words on the paper are some of the distinctive features of physical organization and design and will be analyzed in the rest of this section. All through this novel, there is an ongoing dynamic relationship between the signifying strategies and the physical resources; the physical resources have been purposefully employed to make a design equivalent of the events which are being narrated through the signifying strategies.

\section{Cover}

Reflecting the impossible house within the novel, the front cover of this novel is half an inch smaller than the other pages and its black color and slightly textured surface symbolizes walls within the hallway. The word "house" on the cover, as in all other parts of the novel, is highlighted in blue and is at the top of what can be interpreted as the blueprint of a house on an architectural plan. The addition of the blueprint to the cover opens the door for another interpretation of the word "house" which appears in blue all through the novel. Since Danielewski has never been shy in confessing his love for print and, as he says in an interview by Sophie Cottrell, "books are remarkable constructions with enormous possibilities" (interview by Sophie Cottrell), the term blueprint seems to be the perfect word for a house that can only appear in blue ink on white paper. This is also the main reason that any attempt to find a real-world counterpart for that house would eventually fail (as Johnny says in the introduction as well). Therefore, the word "house" has to appear in color blue, to emphasize its blue print/blueprint status.

The stairway in the blueprint is surrounded by numerous rooms of various sizes. At the center of this stairway is the image of a yellow object which can be interpreted to be a compass. However, the four directions are absent from it. This absence reflects the refusal of the compass to settle on any one direction within the house when Navidson's wife, Karen, later in the novel buys a compass after she 
realizes that "the whole house defies any normal means of determining direction" (Danielewski, House of Leaves 90). The entry to the stairway on this textured surface is toward the right end of the cover, which means in order to enter this huge black space one has to go through the book and start reading it. The last word which appears on the cover is "A Novel" in purple at the bottom of the cover. The reason for the choice of purple for "A Novel" is later analyzed in more detail.

Similar to the expansion of the hallway, the unusually large folded part of the cover-which is three and thee quarter inches wide when it is opened can cover the whole book, and has a blurb about the novel and its story. The spine of the book has six frames, three of which are images of a white house from different views and angles, interspersed with the three other black frames which have the book's name, author's name, and the publisher's name on them. The back cover like almost any other contemporary traditional and experimental novel, contains the excerpts from the reviews of magazines such as New York Times Book Review and Newsweek. In the bottom half of the cover in addition to the name of the publisher and cover designer, in an obvious nod to the novel's attempt to "break out of the cover" and expand into other media, there is the name logo of Danielewski's sister, Poe, along with the address of her website and the name of her second album, Haunted, which was released in same year as the book. This album as we will see later provides another "entry-point into the house."

\section{The First Color Plate and the Title Page}

The first color plate of the novel, part of which is visible from the front cover is a collage composed of various unrelated objects such as compass, bullet, paper excerpts, stamps, etc. which play a direct or indirect role in the novel. The images of these objects, similar to the nude images of the woman in Willie Masters' Lonesome Wife, do not signify anything by themselves, but it is through the words which appear on the following pages of the novel that the significance of each of these objects and its contribution to the narrative becomes more evident. In this sense this collage and the other collages within the novel complement the "index" section which comes at the end of the novel. Here the print novel becomes a frame within which all these assembled elements from different modes work together to create meaning through 
the relationships established among them. In other words, this opening pictorial element at the very beginning of the novel poses a question which the novel as a verbal construct goes on to answer: why have these random objects been put together and what kind of relationships can they have toward each other?

Another textual feature which is worth mentioning here is the way Danielewski has used the two-page spread as a screen in the title page. The top verso page has the author's name along with an apostrophe which is completed by the name of the novel which, following the arrangement of these names in traditional novels is placed at the top of the recto page. This arrangement is the first instance in which materiality of the book, the two pages as a screen, has been used to demonstrate Danielewski's allegiance to print and to indicate the ways in which it is different from the works of traditional print fiction. The title page on the recto page is what one would see in many traditional print novels, that is the title of the novel is printed in bold face and a larger font type at the top of the page, followed by the name of the author below it, and (usually for classic novels) an introduction and notes by an acclaimed scholar in the field. However, here the addition of another name on the verso page with an apostrophe s-which makes the reader look further to find the object that this apostrophe claims the possession of - challenges the whole structure not just at the level of signifying strategies (with questions like whose book is this? If it is written by Zampano, so why does someone else claim to possess it? etc.), but also by treating these two pages as a screen which should be taken together.

At the bottom of the copyright page, which follows this page, there are the words "First Edition" in purple but they have been crossed out. This makes the copyright page fulfill a requirement of the CIP to correctly acknowledge the printed edition and at the same time by having it crossed out makes it not the first edition.

\section{Colors}

The full color edition, as its name indicates, in addition to the traditional black on white has three more colors for words: blue, red and purple. The first color, blue, is consistently used for the word "house" throughout this novel in every language, whether English, French, German or even Latin. This color makes the word "house" stand out in the novel wherever it occurs and makes it opaque and harder to look 
beyond (which is the opposite of the use of language in its everyday usage, which wants to make language transparent). Looking at blue from this angle makes it easier to understand why the vast interior of the house is also opaque and impervious to light. As for choosing the color blue over other colors in this novel, apart from the interpretation that was discussed before, the other possible interpretations for the use of this color is its association with digital textuality. According to Martin Brick:

The association with hypertext suggests an unseen network; that at any moment the reader could click on the word and be transported to another part of the book. Everything is tied and the reader is expected to play an active role in navigating House of Leaves. ("Blueprint(s)")

This unseen network, which according to Brick, is triggered by the presence of the blue color for the house, is part of the media-consciousness that was discussed above. By painting the word house in blue (and as we will see later, purple and red), the novel remediates the physical shape of the networked structure of the Web. This remediation does not merely stop here and as we will see later continues through the narrative strategy and the expected reading process of this work.

The links which appear in blue words in webpages make it possible for a specific document to be linked to network of documents. These blue words are then entrances to other documents which may or may not have other links. Thus they show the infinite space within the house which keeps changing in size and direction. When read on the PDF file that Danielewski had originally uploaded on the Web, the blue color of the word house, plays with the expectations of the medium because it is supposed to be a link whereas it is not, but still it stands out in the text like a link in a HTML-supported browser does.

Although blue has been used extensively in the novel, the color which stands out more than any other in the book is red. According to "A Note On This Edition" this color has been used for the Minotaur and struck-through passages. All the words which appear in red are in the Labyrinth and Minotaur chapters (chapters IX and XIII respectively). A black thread goes through all the red words in the Labyrinth chapter and as Johnny describes in an unnumbered note in the footnote: "the struck passages indicate what Zampano tried to get rid of, but which I, with a little bit of turpentine 
and a good old magnifying glass managed to resurrect" (111). The black thread which goes through all the words in red and even the spaces between them can be the thread which Ariadne gave Theseus to find his way out of the labyrinth after slaying the Minotaur. This thread was the key to defeating the labyrinth and being able to get out of it, and this is why the part of the novel containing the story of Minotaur and the play the fictional playwright Taggert Chiclitz has written about it has the shape of a key in the concrete prose.

It is evident that Zampano was trying to hide these passages and leave them out of the final copy. But Johnny rescues them from oblivion and restores them to the final edition of the House of Leaves. This rescue operation of Johnny mirrors that of Ariadne and this is the reason that a line goes through these rescued words. The magnifier, which has been a key here for Johnny, is the main reason that the key shaped by words is too big for a single page and appears on two pages. The only instances of red words which are not struck through are in chapter XIII, for the word Minotaur. Red, which is a color that most attracts attention, makes the word Minotaur stand out in the page, in the same way that the physical deformity or the strange shape of Minotaur would have made it stand out among others.

As soon as one clicks on a link, it turns red in HTML, and the already visited links appear in purple. So it can be said that blue is the color for the links that you have not visited yet, red is the color for the links which have been activated and finally a visited link appears in purple. The HTML tags provide us with a wealth of possible interpretations for the colors in this work. The house always appears in blue and never turns into purple, because it is never the same. This house as Danielewski has aptly named his novel is a "house of leaves" in which nothing is constant and its walls keep shifting all the time. The red color of Minotaur and the struck through passages means that they are active in the sense that the Minotaur which, as it is explained in one of the footnotes, could have been a deformed child is still active in the form of the digital medium, which has been seen as a threat to the print medium. If we follow Hansen's argument that the house represents digital textuality, it will not be surprising to see why this beast is housed there, and though it can never be seen-which in a way shows the fixture in popular imagination about the immateriality of the digital media-its traces are visible. The color purple in which 
the word "novel" appears on the cover, can be interpreted as the narrative which has been visited by some people (the narrators), and the readers of the first edition (either the ones that Danielewski had posted the link to the PDF version uploaded on the Web, or the ones to whom the first edition of the House of Leaves had been privately distributed, as the 'foreword' of the novel says).

\section{Fonts}

House of Leaves has been typeset in four different fonts which help the reader to determine who is saying what and especially when it comes to the footnotes and the commentary, distinguish between Johnny's posts and the Editors'. As The Editors have stated in footnote number five on page four, "In an effort to limit confusion, Mr. Truant's footnotes will appear in Courier font while Zampano's will appear in Times." Times which is the short form for Times New Roman is now the standard font for MLA and APA style research, and helps to maintain the scholarly appearance of the novel, whereas the Courier one used for Johnny's footnotes makes it quite clear that Johnny is the carrier and deliverer of the story, and the agent who, like the medium, provides a way for Zampano's narrative to be seen and read; however, this courier of the story does not try to be transparent and makes his own adventures a part of the manuscript that he is writing/editing.

"Zampano only wrote "heater." The word "water" back there [in The Navidson Record] - I added that.

Now, there's an admission, eh?

Hey, not fair, you cry.

Hey, hey, fuck you, I say" (16).

Danielewski names the other two fonts in an interview by Eric Wittmershaus: "the title page is Dante and the editors' typeface is Bookman." Dante is the font which Danielewski will use again for his second novel Fifty Year Sword, and was analyzed in the previous case study. The meaning of this font here is the same as the one in the previous case study, which in short is leading the reader through the entrance of the novel, and then leaving her to explore it by herself. However, this font has been used 
here for another part of the novel which has not been discussed by other critics, so paying attention to this font can lead the reader into a more rewarding understanding of the novel and the internal relations within it. The Dante font has been used for Pelafina's letter and this font as we will see later in the narrative strategy can help to explain a variety of other enigmas in the novel.

Bookman as its name shows has been a clever choice for The Editors because they are the ones who participate in writing/editing and publishing the book. Despite Johnny, they do not play a major role in the novel, and their role is mostly tying up the loose ends like providing the translation for the words and sentences for which Johnny has not been able to find the meaning, or has mistranslated.

\section{Footnotes}

Moulthrop in one of his essays, "You Say You Want a Revolution? Hypertext and the Laws of Media" had argued that links are the digital equivalent of the footnotes in print. Danielewski foregrounds the graphic surface of his novel, by bringing in so many footnotes, that his aggressive use of them can be compared to the aggressive use by Joyce of the guard-fields. As we see in the House of Leaves, the footnotes appear on almost every page, and from the beginning they appear in different sizes. For instance, very early in the novel on page twelve, there is a footnote which is four pages long, and then there is another one on page nineteen which is almost as long as the chapter itself. Most of these lengthy footnotes belong to Johnny and these footnotes, while serving their primary role of "author's comment" and "citation of a reference," provide another opportunity for the book to have a parallel layer of narrative within the footnotes. This parallel narrative as we will see in the next section has a dynamic relationship to the other narrative, and both complements and compliments the other narrative. The footnotes also at times abandon their traditional location at the foot of the page, and appear at other parts of the page.

Similar to the experimental placement of the footnotes all over the page, the footnote symbols in several pages of the novel differ from the symbols which are ordinarily used for footnotes. These new symbols can be interpreted as various levels of code which provide another interpretive layer for the text that they are accompanying. One of these codes, which will be explored in the reading process 
section is Ground Air Emergency Codes. The unconventional symbol for the footnote draws attention to itself and makes it stand out on the page to emphasize the importance of the footnotes in this novel and how tracking down the meaning and origin of these symbols can provide another layer of meaning here.

\section{Arrangement of the words on the paper}

Regardless of whether they are footnotes or the main texts, the words have been arranged in a disruptive way that makes them highly visible. The words in this novel follow the narrative that they are representing in their arrangement on the page. There are several pages in this book where only a few words appear at the very bottom or the top or the middle part of the page. However, the experiment with the arrangement of words on the page does not actually end here and the novel showcases a variety of other arrangements, which have been rarely used (if ever used at all) for either the traditional or even many experimental novels. Some of these arrangements would require the book to be turned in different degrees to be read.

For instance, reflecting the comparisons which have been made between two brothers, chapter XI is divided in two columns (for both the main text and the footnotes). The paragraphs in the main text have been divided by one single " $r$ " and more than twenty "z"s which follow it. This strange phrase is explained by the first paragraph on page 250 which supposedly describes the meaning (of fictional) root rzz to be "to tear apart, to shatter." Thus the theme of separation which has been visualized through the separate columns in each page is further emphasized through these "rzzzzzzzzzzzzzzzzzzzzzzzz"s which follow virtually every paragraph.

Another example of the arrangement of words on the paper is when the whole text finds a transcript format in the very next chapter, and the font becomes noticeably larger. This change in font size and the arrangement of the words in the middle of the page shows, Will Navidson's brother, Tom's thinking aloud while he is left in the dark corridor alone.

However, the most ambitious play with the format of the page starts as soon as chapter XII starts, and the main characters (Navidson and Reston) come to the foot of the stairway, the arrangement of the text on each page follows their location in the 
hallway, and when the stairway in the hallway starts stretching, the words follow suit and page 289 is arranged in a way which shows the stretching of the stairs with the letters of each word having a longer than usual distance from each other. To emphasize the feeling of vertigo that Navidson is experiencing, the whole page has been printed upside down and the book has to be turned 180 degrees to be read. This upside down arrangement has been repeated on every other page during the next couple of pages and the reader has to turn the book for reading each page until she comes to the part where the rope which Navidson is hanging from snaps and the character and words describing this part are left hanging in the empty space of the page.

Chapter XX in which Navidson enters the hallway again follows the same unconventional arrangement of the words on the page, and takes this experimentation still further. In order to visually represent the haphazard way the hallway moves and - consequently the disorientations that Navidson experiences - there are slanted, semi-circular, and diagonal lines of sentences on several pages. So by looking at each page, it can be seen where Navidson is and what kind of movement he has had there, or how the hallway is narrowing when it starts to shrink. All these unconventional arrangements provide a visual representation for the novel through the physical resources of what the signifying strategies are representing, and emphasize the graphic surface of the novel.

\section{Narrative Strategy}

There are no descriptions in fiction, there are only constructions. (Gass, Fiction and Figures 17)

My notion of a work of art is not that it is a copy or a representation of reality, but an addition to reality which is intensely human itself. It contains-embodies-human consciousness, presumably at a high level of refinement. What happens with texts which, from my point of view, are successful, is that the language is transformed from a language of utilitarian import to a level of what Rilke called "things." So you're trying to create a language which now 
serves, not as a message bearer, but as an experience, a container of consciousness, in fact. (Gass, interview by Jan Garden Castro)

After all, as I fast discovered, Zampano's entire project is about a film which doesn't even exist. You can look, I have, but no matter how long you search you will never find The Navidson Record in theaters or video stores. Furthermore, most of what's said by famous people has been made up. I tried contacting all of them. Those that took the time to respond told me they had never heard of Will Navidson let alone Zampano. As for the books cited in the footnotes, a good portion of them are fictitious....Add to this my own mistakes (and there's no doubt I am responsible for plenty) as well as those errors Zampano made which I failed to notice or correct, and you'll see why there's suddenly a whole lot here not to take too seriously. (Danielewski, House of Leaves xix)

While enthusiasts and detractors will continue to empty entire dictionaries attempting to describe or deride it, "authenticity" still remains the word most likely to stir a debate....For the most part, skeptics call the whole effort a hoax but grudgingly admit that The Navidson Record is a hoax of exceptional quality....Though many continue to devote substantial time and energy to the antinomies of fact or fiction, representation or artifice, document or prank, as of late the more interesting material dwells exclusively on the interpretation of events within the film. (Danielewski, House of Leaves 3).

House of Leaves [is an example of] work as assemblage, a cluster of related texts that quote, comment upon, amplify, and remediate one another (Hayles, "Translating Media” 278).

As can be seen from the Gass quotation, he supports a non-referential role for the language in fiction so that a work of fiction instead of offering a representation of reality, becomes an addition to it. Danielewski follows the advice of Gass to a fault, 
he foregrounds the graphic surface of his work and at the same time in Pressman's reading of Hansen narrates "a very real void of referentiality." The narrative in House of Leaves is about an impossible house and makes construction a key part of its narrative strategy; therefore, the story of the novel becomes the story of the construction of each layer within the work. This is one of the reasons that Johnny emphasizes the irrelevance of the authenticity and factual value of the work he is editing; he is conscious of the value of the work of art that is being constructed/assembled by him. Similar to Gass's metaphorical statue, it is not the referential value of the narrative that is important but the metatextual one that really matters.

This point is further emphasized by "The Index" which presents words in isolation, and shows how these words when assembled together can construct stories which according to Gass are not a "representation of reality but an addition" to it. Thus, in analyzing the way the narrative strategy of the novel works to highlight the materiality of the medium, the word "construction" becomes a keyword. There is no story out there, and it is the narrative discourse that constructs a story from the pool of stories. Johnny's "House of Leaves" is one of the possible constructions of the story and this is exactly what Johnny hints at by adding manuscript excerpts in the Appendix I-C in which the ending of the movie/story is different from what has been referred to by Zampano and him.

To emphasize the narrative's resistance to representation, Johnny at some point travels to Virginia to find and visit the Ash Tree Lane house which he has been reading about from Zampano's notes, but he does not find anything there:

Everywhere I've gone, there've been hints of Zampano's history, by which I mean Navidson's, without any real evidence to confirm any of it. I've combed through all the streets and fields from Disputanta to Five Forks to as far east as the Isle of Wight, and though I frequently feel close, real close, to something important, in the end I come away with nothing. (501)

The other support for this argument is Zampano's blindness. Since he is "blind as a bat", logically he could not have watched the movie, let alone having written a 
detailed analysis of it frame by frame. Looking at it from this angle would make it no surprise that there is no such movie, no such house and no such tattoo shop. Johnny has shown his skills in constructing narratives, in the beginning of the novel when he talks about his scars and the cage fighting, and the visit to his GP friends at the end. The theme of narrative as construction runs through every level of the narrative. In "The Navidson Record" the characters are trying to (re)construct what they have not seen or have missed due to their absence or their ignorance. The movie is trying to capture the objective reality, but as we see, Navidson by his ingenious edit of the movie constructs the narrative that he wants to make, which is a love story (as discussed by many fictional and real critics), and highlights one particular part of that construction. Later, Karen herself constructs a movie ("A Brief History of Who I Love") which results in her re-discovery of her love for Navidson. Although Navidson has been there the whole time, she constructs the Will Navidson that she loves and is worth rescuing through her movie. Similar to the index, there are vast amounts of takes/rushes which can be put together to various effects, and it is through the process of editing that these "takes" construct a narrative. The same rushes, within the hand of another editor could result in a totally different film with the theme of horror, rather than love.

Zampano takes the whole concept of "narrative as construction" to another level by mixing the real and the fictional sources for the pseudo-academic text that he is creating. Meanwhile his creation is two-fold. He is creating the narrative for "The Navidson Record", and at the same time he creates another academic narrative/commentary for "The Navidson Record." In the same way that the takes/rushes of what later comes to be known as the film, "The Navidson Record" are put together/edited to create a film, Zampano leaves behind a vast accumulation of notes which as we will see another writer/editor has to arrange to create a narrative, The Navidson Record, out of them. The arbitrariness of this construction/assemblage is further highlighted by the contents of the appendices; Zampano constructs his commentary out of the numerous books that he quotes from or comments upon. All those books laid beside each other could not create a commentary, but it is Zampano who starts his quest of writing a commentary for the fictional documentary. $\mathrm{He}$ emphasizes the fictionality and the "constructed" nature of his work by quoting from 
fictional works some of which have such funny/extraordinary names that they stand out where they appear (either in footnotes or endnotes).

Johnny, on the other hand, has no qualms about the fictionality of the whole text that is in front of him in literally several bits and pieces. So he constructs three texts at once, The Navidson Record, the accompanying commentary of Zampano, and his own footnotes from the pile of material which has been laid before him.

This task becomes even bigger for the Editors who have to create four texts by adding their own input in the form of commentary on Johnny's commentaries which (at least half of them) are themselves comments on Zampano's writing, which in itself is a commentary on Navidson's creation. The role of The Editors is itself two fold; making corrections to the text that they are editing/constructing, and selecting the formal elements for this book. These formal elements range from the font types and their sizes to the inclusion of the appendices and the index, acknowledgement page etc. to the novel. Any of these choices directly affects the narrative and the way it is constructed within the novel.

The dynamic layering aspect of the narrative strategy appears in the way that each embedded narrative tries to undermine the one before it, and this process becomes a never-ending loop when Will Navidson burns the copy of House of Leaves that he is reading, and when Johnny finds a printout copy of the House of Leaves, while he is still working on it and has kept it in the trunk of his car. This conflict of narratives for dominance - "the" story of the book - is both supported and rejected by the additional resources within the book and beyond it.

The search engine of the novel comes in the form of the index at the end of the book. This index which is over forty pages long, like a search engine provides a word search, rather than the usual index selection of topic and personal names is a unique feature of this novel. However, this is no ordinary index and several entries have been marked DNE. This acronym can stand "Does Not Exist." Although these entries undermine the reason for their own existence within the entry, their placement here remediates the dead links in electronic textuality. In other words, DNE is the print equivalent of a dead link, because it points toward something which might have been, but does not exist at the moment. Finally the overall issue is that although the book is 
a physically bounded entity/object, the boundaries of the narratives contained within it are not co-extensive with the book itself, or the sequence of its pagination, and this makes it similar to a narrative on the Web.

As already discussed, there are multiple layers within the narrative (The movie, Zampano's writing about the movie, Johnny's construction/assemblage, the Editors' notes, Pelafina's letters, etc.). In order to create each layer, the narrative has gone through a process of selection which includes several adding/removing stages (or more simply put, several edits). It is only after these edits that the final cut of the movie/book/narrative is prepared. Meanwhile, someone other than Zampano, Johnny, the Editors, or Dr. Whyrta could/might have chosen the left-out frames/scraps and have come up with a different narrative. The presence of "end matter" for the House of Leaves also makes this construction process more complex, because the narrative can be constructed out of any number of combinations between the references and the their referents within the text. A good example for this is Pelafina's letters which, as we will see in the next paragraphs, Jessica Pressman comments upon in her essay. The same goes for the real life reader/editor as well, since the book expands beyond itself into the forum designed for the novel and the music album, there are several narrative clues available outside the book, and it is through each narrative discourse that a different form of narrative is unfolded.

Jessica Pressman, who does go beyond the book into the music album, and the forum finds that how reading this novel across its network "helps build a theory of authorship for the narratives." Pressman in her essay "House of leaves: Reading the Networked Novel" analyzes the song "Five and a Half Minute Hallway"-which is both a song played by the band Liberty Bell in the narrative of the novel, and a song in Poe's album - the online forum for the novel and the book Whalestoe Letters.

The lyrics of Poe's song open up a new dimension of interpretation for her, and she links them to the traumatic experience that Johnny has had as a child which lasted exactly five and a half minutes. After discussing several other clues such as the date of the last journal entry by Johnny, the date of the conclusion of The Navidson Record and the release date of Poe's album Haunted and how all of these happen on a Halloween, she moves on to Haunted, and Whalestoe Letters to see whether she can 
find out more about the story. In the same song, there is a line which says, "But there's more to this story/ Than I have exposed/ There are words made of letters/ Unwritten" (italics mine). As she aptly identifies in her essay, the editor of Whalestoe Letters, Walden Wyrtha (whose attention to the letters has been triggered by his wife Waheeda) through his strange name makes it possible to make the acronym of WWW from his name and his wife's name. Dr. Wyrtha further encourages this relationship by stating that "I was and am today an Information Specialist which ... is just a fancy title for one able to organize, catalogue, index and cross-index" (xi). In that book, Pressman finds that the eleven letters which are not in the novel "shed light on the congruence between the author of these letters and the author of the narrative layers in the novel" (116). For instance, they follow the same "typographic experiments" of the novel, or the doll in Zampano's The Navidson Record is exactly the same doll that she has described. It is after bringing all these clues together that Pressman claims that Pelafina is most likely candidate for the narrator and the writer of the narratives in House of Leaves.

Another support for Pressman's claim, which was hinted at in the previous section, is the font type used for Pelafina's letter. A simple comparison between the font type of this part with the font type of the other parts shows that the same Dante font which had been used for the title page is used for Pelafina's letters as well. It is also possible to find another support for Pressman's discussion from Danielewski's interview by Sophie Cottrell: "These days though, I like to look at House of Leaves as a three character play: a blind old man, a young man, and a very special, extraordinarily gifted woman. The three of them are telling each other stories" (Danielewski, interview by Sophie Cottrell) The blind old man is obviously Zampano, a young man can be Will or Johnny, but since Will is not into explicitly telling stories - he wants to record rather than write-Johnny becomes a better candidate for the young man. Apart from Karen and Pelafina, there are no other major women characters in the novel, and as far as the narrative goes Karen would not qualify as a “very special, extraordinarily gifted woman" whereas Pelafina's insanity and her extraordinary imagination-which is shown through her letters to Johnny and particularly the layered one on page 627-makes her a "special" woman. 


\section{Reading Process}

As is true of hypertexts and other forms of electronic writing, the novel isn't arranged linearly or hierarchically-that is, what appears in the Appendix isn't necessarily of lesser significance than what you find in the "main part" of the book, and isn't even necessarily supposed to be read afterwards. (McCaffery, "Haunted House"123 )

Before analyzing the reading process of this novel, it is necessary to find out who will be its ideal reader according to the novel itself, or in other words how does this text expect to be read. In order to find the answer to this question, I will have a look at the examples of readers reading the novel here, and then I will examine how these examples guide the reader in understanding the purposes of the text.

As was discussed in the Narrative Strategy section, there are several readers/writers at work in this novel. The first one is Zampano whose House of Leaves is both a commentary on and a description of "The Navidson Record." There are two aspects in Zampano's reading of "The Navidson Record," which make him an ideal audience/reader. Zampano keeps writing his comments for "The Navidson Record" on whatever material he can find. Since it is impossible to literally write on the VHS cassettes through which "The Navidson Record" has been released, he writes a description of the movie and creates a verbal representation of that and then adds his own comments. In other words, Zampano's writing can be divided in two parts which progress hand in hand together. In one part he is remediating "The Navidson Record" in print, and in the second part he is adding his own commentary to that. As we will see below, in remediating the movie through print, he has tried to preserve the dynamic relationship between the physical resources and the signifying strategies that had identified in the movie:

Navidson names this sequence SOS which aside from referring to the distress signal sent by Halloway's team also informs another aspect of the work. At the same time he was mapping out the personal and domestic tension escalating in the house, Navidson was also editing the footage in accordance to a very specific cadence. Tasha K. Wheelston was the first to discover this carefully created structure: 
At first I thought I was seeing things but after I watched SOS more carefully, I realized it was true: Navdison had not just filmed the distress call, he had literally incorporated it into the sequence. Observe how Navidson alternates between three shots with short durations and three shots with longer durations. He begins with three quick angles of Reston, followed by three long shots of the living room (and these are in fact just that - long shot taken from the

foyer), followed again by three short shots and so on. Content has on a few occasions interfered with the rhythm but the pattern of three-short three-long is unmistakable. (102)

In arranging this part on the page, Zampano has divided his own writing into three paragraphs which start and end according to their own lengths, not the grammatical structure of the sentences. Another aspect of Zampano which makes him an ideal reader/audience for "The Navidson Record" and can be observed in the above quotation is that he is quite well-informed about the literature surrounding the "The Navidson Record," and he seems to have read almost all of the books/essays which have been written about it. In other words he has searched for (and found) all the possible resources which help him to have a better understanding of the contents and stylistic plays of The Navidson Record.

The second writer/editor who has a big role within the narrative, and the narrative moves forward through his reading of Zampano's writing, is Johnny Truant. He produces a large amount of commentary on Zampano's The Navidson Record and as he says in the introduction to the novel he has tried to find the movie and the books which Zampano has referred to in his House of Leaves:

After all, as I fast discovered, Zampano's entire project is about a film which doesn't even exist. You can look, I have, but no matter how long 
you search you will never find The Navidson Record in theatres or video stores. Furthermore, most of what's said by famous people has been made up. I tried contacting all of them. Those that took the time to respond told me they had never heard of Will Navidson let alone Zampano. As for the books cited in the footnotes, a good portion of them are fictitious. For instance, Gavin Young's Shots In The Dark doesn't exist nor does The Works of Hubert Howe Bancroft, Volume $\underline{\text { XXVIII. }}(\mathrm{xx})$

Johnny Truant as we can see from the above examples is a writer/editor who like Zampano comments on what he is reading and he also tries to find the "The Navidson Record" and the books/essays/interviews which have been written about it. He also tries to follow the dynamic relation between signifying strategies and physical resources which can be seen in Zampano's The Navidson Record in his own "House of Leaves." Thus, Johnny, like Zampano, conducts a reading which involves both commenting on and searching for the literature about it.

The third reader/editor who officially wears the mantle of The Editor(s), also follows the same tracks in her reading and in fulfilling the traditional role of the editors of print books, adds minimal additional information to what is already there, and in searching for the books and essays referred to within the novel, she finds out that The Works of Hubert Howe Bancroft, Volume XXVIII does exist and they scan the first page of that book which has been borrowed from the Beverly Hills Public Library and add it to Appendix III. A good scale for comparing the ratio of Johnny Truant's input to the text that he has been reading/editing, to the text The Editors are reading/editing is comparing the lengthy introduction which Johnny has written for Zampano's House of Leaves, with the short "foreword" section which the editors have written and has only 3 sentences.

What can be inferred from all these examples of the reader reading in the novel is that this novel requires the reader to perform these two tasks during her reading process. The first thing that a reader should do is analyze it very closely and add her own commentary to the novel by literally writing in the margins of the novel in the same way that the musicians whom Johnny happens to listen to at the end of his 
journey have done. As it is stated on page 514, Johnny cherishes "the substance of those pages" which have been "marked, stained and red-lined with inquiring and... frequently inspired comments" and he becomes amazed when he sees that "in a few of the margins, there are even some pretty stunning personal riffs about the lives of the musicians themselves" (514). The reason for all this is that Johnny here has met the ideal readers for his work who have done the same thing that both himself and Zampano have done before. This evidence also supports the claim for the ideal reader which has been made above.

As far as the material on House of Leaves goes, there is no shortage of things to be read/listened to. Haunted by Danielewski's sister Poe, as discussed above, is a "counterpart" to Danielewski's House of Leaves and we have already seen what a huge contribution it can make to the understanding of the novel. There are also several websites like "TV Trope" and "The Idiot's Guide to House of Leaves", which offer explanations and clarification for some of the issues which the House of Leaves raises (such as Who wrote House of Leaves, the Latin phrase which Johnny approximates at the end of footnote seventy seven, etc.). The forum of the novel includes a great many contributions from over 90,000 members who have offered possible interpretations to the "tricks" of the House of Leaves.

This novel from the very beginning informs the reader that there is a variety of ways to read it. "The Note On This Edition" which differentiates four different editions brings the materiality of the print novel to the foreground and makes the reader go through the book she has at hand in order to see the features which her copy is supposed to have, and how this one differs from the other versions. Here this text emphasizes the fact that this particular novel is not trying to make the reader forget the medium through which the narrative is being presented, but it is actually making its medium of presentation play a significant role in this process.

The reader of this novel can hardly avoid the networked structure of the novel which imposes itself on readers from the very first footnote. As noted above, Stuart Moulthrop has observed that the links are dynamic footnotes. Links in works of hypertext and web-fiction provided different ways to read a novel. Here the footnotes do the same thing and provide the reader with several reading processes which as we 
will see later keep crossing each other at crucial points in the book. In the first process the reader can ignore them and continue reading to the end of the chapter and then return to the beginning of the chapter and start reading the footnotes. In the second process the reader can stop reading, either as soon as a footnote appears, or when the sentence or phrase which contains the footnote ends, and read the related footnote. The third process which actually branches out of the second process is continuing with the second process and then stopping reading the The Navidson Record as soon as the footnotes which refer the reader to the Appendices and Exhibits appear. If the reader follows this process, she might spend a great deal of time at the end of the book as soon as footnote \#78 appears on page 72, which recommends the readers "who feel they would profit from a better understanding of his past" to "proceed ahead and read his father's obituary in Appendix II-D as well as those letters written by his institutionalized mother in Appendix II-E." This reading process makes the readers see Johnny from a different perspective. So, in reading this novel, the reader has to make decisions at various points and this process of making decisions becomes even more complicated when footnotes within footnotes appear or when the footnotes refer to footnotes several pages ahead (footnote number thirteen on page nine refers the reader to footnote number sixty seven on page fifty five). The longer the footnotes become, the more complicated this process become and this happens very early in the text (footnote number eighteen on page twelve is extended over four pages), and finally takes over the page and pushes the text to the center of the page.

On the pages of this novel, the physical resources imitate the signifying strategies and by making the reader turn the book in different directions to be able to read it, the book wants the reader to experience the disorientations that the main character(s) are experiencing. This is one of the most emphatic presences of the print medium in fiction, because the medium is openly inviting the reader to interact with it. The only interaction which a reader is expected to have with a work of traditional print fiction is turning over the pages, but here the reader has to literally turn the book around to read the parts which are printed upside down or diagonally.

As was discussed before, the three different fonts which appear alongside each other on many pages within the novel also foreground the different layers of the text and it won't take long before she realizes how much each layer has affected the layer 
below it and how each representation is an idiosyncratic representation/modification of the one below it. Whatever "The Navidson Record" has been, we only see Zampano's, representation of it (and this difference in representation is emphasized in the Appendix I-C where there is a piece of writing which shows an alternative ending to The Navidson Record). Despite Zampano who never claims to have changed anything in his representation of The Navidson Record, Johnny openly claims that he has modified both deliberately or accidentally (the water heater, and ink spillage) what Zampano has written. The Editors' modifications to the text are not evident, as their input has been in providing translations for some of the parts which have been written in foreign languages or having very short comments on a few of Johnny's footnotes. These three layers show how intensively the signifying strategies have been changed in the different representations and remediations.

One of the popular topics of discussion in the House of Leaves forum is finding out the codes and hidden messages which the author has left in his novel. The reading process of this novel can be overwhelming in the beginning for the reader, because there is so much material there that might seem peripheral in the beginning, but as the reader continues her reading she realizes that those materials are pertinent to the narrative and they require a closer attention if their relevance is not evident at first. The reader of this novel, instead of relying on some of the accepted traditions of novel writing/publishing, such as the paragraph breaks, perfectly equal margins on each page, the rectangular blocks of words, finds a novel which defies all of them, and creates a text which actively incorporates the medium and acknowledges its presence all through the novel. Therefore, the reader of this text has to keep an open eye for the slightest clue in the ordinarily-overlooked clues, because all of this tinkering with the materiality of the text has been done for a purpose.

One of the examples here is the way Ground Air Emergency Codes have been used to mark the footnotes within the novel. The presence of these codes as physical resources has been discussed in the first section; here their relationship to narrative and how they enrich the reading experienced is analyzed. These codes have been included in the opening collage of the novel and also in the first of the two collages in Appendix II-C. The first usage of them as a footnote in the novel does not appear until page sixty two. The paragraph which this symbol appears at its end is about Karen's 
sexual frustration, and the footnote at the bottom of the page refers to the guide books for sex which Navidson and Karen have in their library. The reader will soon realize that the first book referred to here can be a clue to what this symbol and the other ones which appear in the next pages could signify. The book in this footnote is Erica Jong's erotic novel Fear of Flying, which not accidentally is about sexually frustrated wives. However, the name "flying" in the name of the book can be a clue for what that specific symbol which has been used for this footnote stands. That symbol according to the Ground Air Emergency Code is used for "aircraft badly damaged." This symbol will be more familiar for the reader if she has had a good look at the opening collage of the first page. 

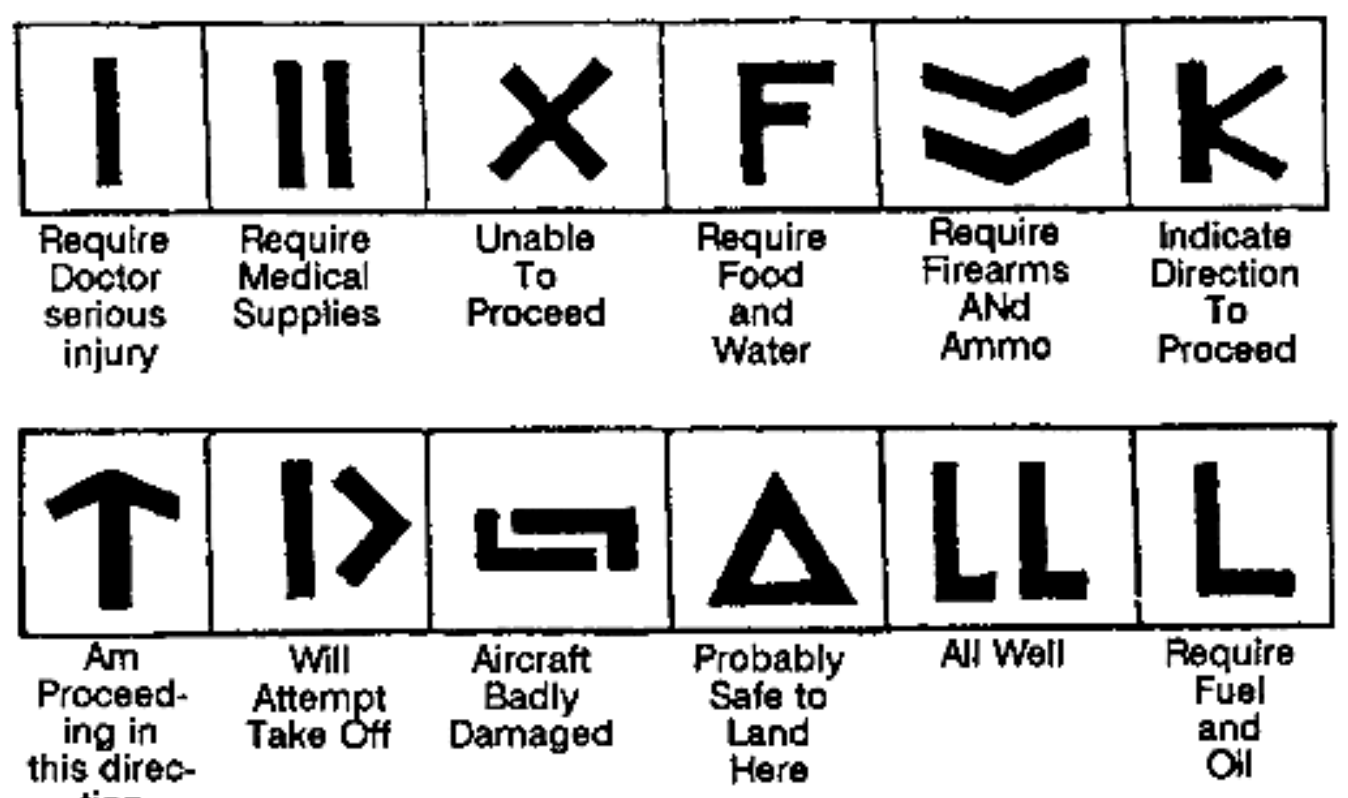

tion

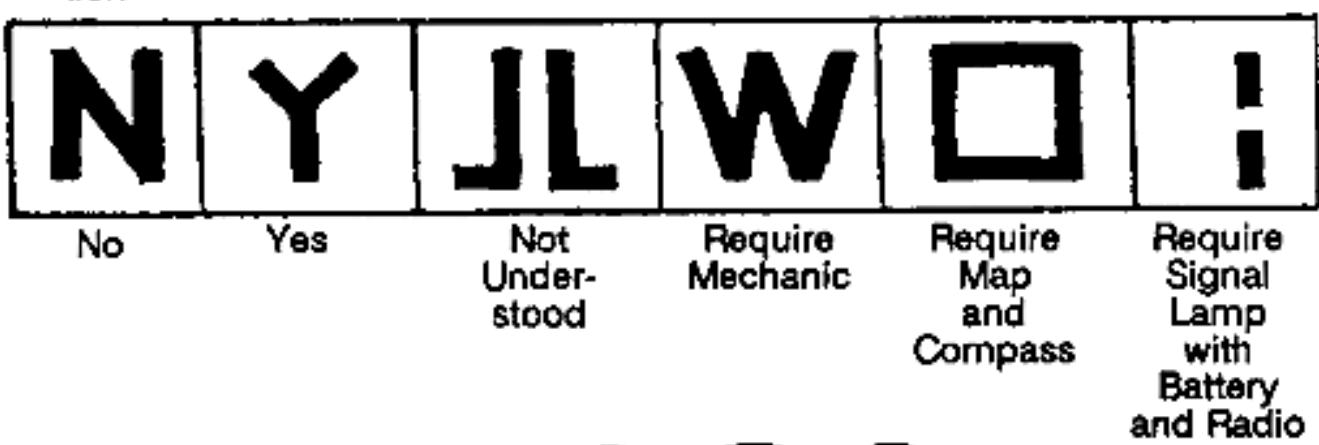

If in doubt, use international symbol

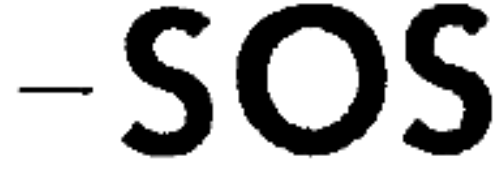

Figure 4.1. Ground Air Emergency Codes taken from https://rdl.train.army.mil.

The second symbol of GAEC is used on page 107 for the translation of the Latin phrases from several authors about the labyrinth. The symbol here which is similar to the capital X, in GAEC means "unable to proceed." Two of the quotations 
here are translated as "The house difficult of exit" and "difficult to enter", and the other one refers to "inextricable wandering." These translations taken along with the GAEC symbol signify that not only it is difficult to enter and exit a labyrinth, it is impossible to proceed within it as well, because there are so many dead-ends and circular routes that someone who has been trapped there might get frustrated and stop moving at some stage. This meaning also foreshadows what happens to Navidson when he gets stuck in the hallway and stops proceeding in chapter XX.

The next code appears on page 109 next to the word bauplan in italics which is a term borrowed from biology. Next to this symbol in the footnote section of the page are the words "so sorry." Taken by itself, this footnote could imply that bauplan means so sorry. Whereas the meaning of this symbol is "not understood" and this explain why Johnny says "so sorry."

The fourth code used here is a small square which appears when the Halloway team start Exploration \#4 and the related footnote discusses the metaphorical meanings of threads and line. The Halloway team are trying to "mark their path" and the code used here which means "require maps and compass" summarizes their efforts here.

The other code is in the form of " $F$ " which stands for "require food and water" comes when the beast within the hallway has devoured the food supply of Halloway team and its placement here shows how they will be in desperate need of food and water later in the exploration.

The next code in this chapter is the same as the second one which has been discussed above. This footnote (in the form of "X") which appears at the end of footnote \#155 on page 123, refers the reader back to the very beginning of this chapter, where this footnote has first appeared, and the meaning of this code, "unable to proceed" finds a different turn here, because this time it refers to the footnote and the maze-like structure of the chapter itself where following some footnotes could take you out of it but following others leads you to dead-ends.

Finally another code from GAEC has been used at the very end of Chapter IX, where Jed and Wax have split from a deranged Halloway and are sitting in small 
room when they hear a hammering on the door (which later appears to be Navidson and Billy Reston, who have come to their rescue). Since these are the last moments of their entrapment in the labyrinth of the hallway, the code which appears there is the "will attempt take off” which again foreshadows what will happen to them.

\section{Conclusion}

As was discussed in the beginning of this chapter one of the most visible effects of digital technology on print fiction is the way that it has facilitated more diverse forms of writing, editing, and page design. The case studies show how each of these aspects have been utilized in a single work and how the digital media as an alternative material support for the novel has affected its development. House of Leaves become a multimedia/multimodal novel in the very literal meaning of the word, because it uses a variety of modes within its cover and takes its narrative to the other media as well.

The representation of different media forms in the print novel shows that the print novel as a narrative media form has diverse potentialities which can be taken advantage of in postmodern fiction. In order to bring these potentials into play, a writer has to have a full awareness of the range of capabilities that print media. Such a writer then can foreground the materiality of his work by interlinking the signifying strategies and the physical resources of the media modalities that he is drawing on to create his work in print.

A reader of such novels has to actively engage with the materiality of the novel and play a more active role than the reader of a traditional novel. Such a reader in her engagement with the medium would try to play the games/solve the riddles which have been predesigned for her in the book and would soon realize that there is no single answer/solution to these games/riddles, and the deep engagement between physical resources and signifying strategies has resulted in texts with many doors, rooms and hallways, where each reader's experience in playing those games or solving those riddles would be way more different than what they may experience from a traditional work of print fiction. 


\section{Conclusion}

From an aesthetic standpoint, the idea of a migration toward the virtual ... has the advantage of letting us recognize the centrality of certain narrative features that have long been marginalized or forgotten. Features that tend to be associated with avant garde or experimental texts-reflexive identity formations, intertextual collaborations, nonlinear or multilinear narrative action, concretism, and the involvement of reader in the act of creation — can again be seen to have been always present in print texts of any literary interest. (Tabbi 120)

The material turn in literature can be traced back to the metatextual works which Kaufmann has analyzed in his book. When it comes to contemporary literature, digital technologies by providing a material support for novel writing have challenged the traditional association of the novel with print. However, in doing that they build upon the metatextual print novels which had made their own material support, that is, print a factor in their narrative. Willie Masters' Lonesome Wife is an early pre-digital example of these works which uses a variety of graphic devices in its presentation and in which design of the novel's graphic surface anticipates future developments through digital media.. Despite the bold claims of some of the early critics that hypertext would bring about the end of the print book, hypertext novelists were more conservative in creating their novels and as afternoon: a story, the earliest and most discussed hypertext novel of this era shows, their works remediated many of the features of print fiction. Experimentation with the materiality of the medium became more radical when the Internet provided a networked structure for computers through the World Wide Web. At the same time, the developments in computer technology made it possible to create multimodal/multimedia works which incorporated a variety of modes/media. These novels challenged the dominance of written language in the print novel and created narratives which mixed these modes/media in new combinations. This phase brought about a new media consciousness for experimental novelists which encouraged them to experiment further with the materiality of the print medium, creating metatextual novels which remediate digital media in print. 


\section{Appendix}

Interview with Lawrence Levy, designer of Willie Masters' Lonesome Wife

This interview was conducted by Mehdy Sedaghat Payam on 11/02/2011 by means of email questions which received written responses.

\section{A. Design issues}

1. Willie Masters' Lonesome Wife has been described as relying on 'radically experimental techniques in typography'. What would you describe as the radical techniques, at the time of creation of the book?

LEVY: I'm not sure I would use the word "Radical" myself. But the use of distorted typography, a variety of typefaces, nude photographs, different color paper stock... which calls attention to the book as object, a metaphor for the body of Willie Masters' wife. In that sense perhaps it was a radical presentation of the written word.

2. What kind of opportunities and challenges did the manuscript present to you?

LEVY: After reviewing the manuscript and then consulting with William Gass, we wanted to give it a new and hopefully pleasing meaning in a contemporary book design...creating a reading experience that goes beyond reading words on a page (not that there's anything wrong with that!)... and gives the words a physical presence that reflects the novella.

An additional challenge was trying to understand the ideas originally presented in the Gass ms. and incorporating their intention, if not always their actual look.

3. Did you plan your design throughout as though the two pages of each opening were a single screen surface on which to display the typographical and pictorial components?

LEVY: No, only the opening (blue) section begins with a 2-page spread. Other sections begin on the right-hand page. 
4. How important in your conception of the book was the notion that a work of art is itself a new object in the world, an object made out of language (and ink and paper) in the case of the novel?

LEVY: That was the heart of it all. The physical embodiment of the words on the page, the body of Willie Masters' wife...coffee stains to remind us that this is a physical object...rings on her "belly where men have set down drinks."

\section{B. Reader expectations}

5. Since most readers would approach a novel expecting narration, did your design aim to take this expectation into account, or to frustrate it in order to draw attention to other dimensions of novelistic fiction? What specific effects was the design intended to have on the reader?

LEVY: Playfulness, sensuousness, all the while saying to the reader that this is more than a neutral repository of words, that this is a physical object (again, see the coffee stains, the colored paper, the different typefaces...)

\section{Financial and Technical}

6. Were there any major components in your initial conception of the design which had to be dropped because of cost or technical difficulty?

LEVY: As a book designer, and also the art director of TriQuarterly, I was experienced in the mechanics of typography and printing and binding, and therefore approached the design conception of Willie Masters' Lonesome Wife within the parameters of cost and technical difficulty in order to avoid Bill and me getting excited about a concept only to have to shoot it down when the estimates came in. There were ideas we talked about (the condom, for instance), but were too expensive to include. 
7. Apart from the bookmark condom, and cloth tip-ins, do you remember any other "nutty" [Gass' word] ideas that Gass had for the book and had to be abandoned due to technical or financial problems?

LEVY: I don't. Did you know that we had each copy of the original 500-copy hard cover edition wrapped in a plain, brown wrapper (as though it was pornography)...and actually sent them out that way? I have several of those still wrapped. I wonder what the books will be like when and if I open them...

8. Why was the novel published as a TriQuarterly supplement [since it was subsequently published by Knopf]. Was this a response to its experimental qualities?

LEVY: It was first published as a TQ supplement. Subscribers received the paperback with their subscription. We also published an edition of 500 hardcover copies, 50 of which were signed and numbered by William Gass. Knopf published a version subsequently....though not as lavishly. Gass was quite well known in 1970 and this was I believe quite an experimental work...both reasons for Knopf's publication. (I'm believe that Knopf only decided to publish it after the TQ edition was out, but I don't really remember.)

9. Did Knopf republish Willie Masters' Lonesome Wife because of its interest in innovative book design?

LEVY: I would like to think the design had something to do with it. [The TriQuarterly hardover edition was selected as an American Institute of Graphic Arts 50 Books of the Year award....which was then the highest American book design award.] Still, I don't think they published it because of the design. It was a great novella before I put my hands on it. Hopefully the look of the book was connected to its meaning, which was the point of the design.

D. Typographic 
10. Gass refers to his plan to use 19 fonts in the printing of the novel, and that using fewer was related to cost. Did you and he share the same conception of the communicative and aesthetic purposes of the typographical design of the novel?

LEVY: I think so...though I took it in a completely new direction, it was always with communication with him and his input. Using additional typefaces didn't have much to do with cost.

Additional note: The typography has to be looked at in terms of the period that it was designed, about 1969. Today, there are far more tools available to the designer composing type with computer programs. Willie Masters' Lonesome Wife relied on analogue optical distortions and other processes including cutting and pasting reproduction prints (called repro prints) of the type. Camera-ready (for the printer's camera) paste-ups of each page were delivered to the printer...not a computer file. In a way, some of the pages appear a bit naïve to me as I look at them in early 2011.

11. How significant in your design is a conception of the written voice, i.e. typography as simulating vocal qualities or giving visual representation to the movement of sound/speech?

LEVY: Not to a large degree. Maybe a few places.

12. There is a clear relation established between font and voice, which would have been complete if every quotation from another author had been represented by a distinct font. What would have been the effect, if this had been possible?

LEVY: I think it would have seemed too mechanical.

13. Does Willie Masters' Lonesome Wife represent a deliberate challenge to the conventional typographical form of the novel, for example does its design represent a refusal of the conventional association between intelligibility, rationality and geometrical regularity, or in other words, the association of written/printed language with mind rather than body? 
LEVY: Not a direct attempt to challenge... but rather to expand... some of the pages or sections look like a conventional novel...others push out in different ways...

\section{E. Pictorial}

14. Gass in a letter to Wolfshohl says: "Levy was wonderful. I still object to the nude. She is far too young and pleasant looking. Otherwise, they did about as well as they could in realizing my ideas, though many of them had to be given up." Did you choose the model?

LEVY: Yes, together with the photographer, Burton Rudman. We shot all the photos in my studio.

Did she represent how you had imagined the central character of the story "Babs" would look if she were real?

LEVY: Yes.

Was this choice discussed between you and Gass before publication, and how if it was, was the difference resolved?

LEVY: I don't remember that we discussed the look of the model...though he certainly saw the pictures and how I intended to use them.

15. Are the placement of the images a design question or was it Gass' idea to place them where they currently are?

LEVY: A design issue...related to the text, of course.

\section{F. Color}

16. Were the page colors exactly the way William Gass wanted them to be or were they significantly changed due to the limitations of printing? 
LEVY: I came up with that, and also, remember, the sections had to be in multiples of 4's or 8's for printing purposes.

What are the exact names for those colors?

LEVY: These were color paper stocks. The color wasn't printed. The book was all printed in black-and-white ink, except for the brown coffee rings on the last two pages.

Was the original text intended to have four different colors, or, like the matter of the fonts, was a wide range of colors initially considered?

17. In On Being Blue, Gass discusses colors and values, were these ideas influential for the choice of the different colors and the papers in TriQuarterly edition?

LEVY: Not to my recollection.

G. Publishing issues

18. As Gass has said in an interview with Paris Daily and a letter to Clarence Wolfshohl, he has not been able to use many of his ideas for this book, in his own words: "and the work also had to go through so many hands, that the visual business was only occasionally successful, and most of that was due to the excellent design work of Larry Levy, not me. Too many of my ideas turned out to be only ideas..." The design of WMLW makes it unusually clear that book publishing is a collaborative process. What do you think of this collaboration? How different was it from the previous and succeeding books which you designed?

LEVY: I have worked closely with authors on several essentially innovative typographic books which had unique design problems (like Charles Newman's A Child's History of America). For the most part, books still don't do much of what Gass was trying to do, and still look pretty straight forward...except in the case of art and photo books, of course. Then there's the whole area of the art book, where the whole text and design (and often production) is done by a single artist. See the 
great selection of work at Printed Matter bookstore in New York (www.printedmatter.org)

19. In the above quote, he vividly praises your design work for the book. Which aspect of your design made him quite happy and satisfied (if he ever was) and did you make any changes to the book that were not included in the manuscript, but were originally your ideas?

LEVY: As stated above, most of the design ideas, including the photography, that are in the present book were developed by me in close consultation with Gass, but were significant departures from the manuscript.

\section{H. New Media}

20. Do you think that technological limitations (the "physical feasibility" that Gass mentions as the second biggest obstacle for producing his book) are any longer important factors if you were to engage in another project of this kind now (at the beginning of 2011)?

LEVY: Newer digital technology makes a book like this (I mean, looking exactly the same) much simpler and less costly.

21. Have you by any chance seen/read any of the works of hypertext fiction? If yes, how similar do you think they are to Willie Masters' Lonesome Wife?

LEVY: I'm just starting to read some hypertext fiction.

22. Are you familiar with Eastgate Systems as a digital publisher of hypertext fiction? Do you see any similarities between TriQuarterly and Eastgate Systems in the interest in innovative fiction publishing?

LEVY: TriQuarterly went out of business, and I hear Eastgate is on the way. TriQuarterly came back to life recently as an internet magazine (www.triquarterly.org). I don't know a lot of what their goals are beyond the 
traditional literary magazine, albeit on line rather than on paper. Certainly the internet brings the possibilities of all sorts of innovative fiction, interactive and otherwise, to a new level...though I still love to hold an actual book, smell the ink, turn the pages...even put my coffee cup down on it...leaving a stain which reminds me that I spent some enjoyable minutes reading those words...

23. Finally, do you see a close relationship between film-making and book production?

LEVY: Certainly...I moved in my career from book designer to film maker. I think there are a lot of similarities...though I think those similarities may only be obvious to one who knows both areas: you start with a manuscript, or a script...cast it with typography and pictures, or actors....print it on paper, or shoot it on film....many similarities in the process, the collaborations, the budgeting. I don't want to push the analogy too hard. 


\section{Bibliography:}

1001 Nights. Sterling Publishing Company, Inc., 2008. Print.

Aarseth, Espen J. Cybertext: Perspectives on Ergodic Literature. Baltimore, Md.: Johns Hopkins University Press, 1997. Print.

Abbott, H. Porter. The Cambridge Introduction to Narrative. Cambridge, UK ; New York, NY, USA: Cambridge University Press, 2002. Print.

Abrams, M. H, and Geoffrey Galt Harpham. A Glossary of Literary Terms. Boston, Mass: Wadsworth, 2012. Print.

Bantock, Nick. The Artful Dodger: Images \& Reflections. San Francisco: Chronicle Books, 2000. Print.

---. The Forgetting Room: a Fiction. New York, NY: Collins Publishers, 1997. Print.

---. Griffin \& Sabine: 20 Assorted Notecards \& Envelopes/Cards. Chronicle Books Llc, 1994. Print.

---. Interview by Gavin J. Grant. IndieBound.org. n.d. Web. 25 Nov. 2013.

---. Urgent 2nd Class: Creating Curious Collage, Dubious Documents, and Other Art from Ephemera. San Francisco: Chronicle Books, 2004. Print.

Barthes, Roland. The pleasure of the text. New York: Hill and Wang, 1975. Print.

Beiguelman, Giselle, and Helga Stein. Code Movie 1. Electronic Literature Collection Volume One, 2006. Web.

Bell, Alice. The Possible Worlds of Hypertext Fiction. Houndmills, Basingstoke, Hampshire; New York: Palgrave Macmillan, 2010. Print.

Bennett, Tony, and Patrick Joyce. Material Powers: Cultural Studies, History and the Material Turn. Routledge, 2013. Print.

Bernstein, Mark. "Patterns of Hypertext." Proceedings of the Ninth ACM Conference on Hypertext and Hypermedia : Links, Objects, Time and Space-structure in Hypermedia Systems: Links, Objects, Time and Space—structure in Hypermedia Systems. New York, NY, USA: ACM, 1998. 21-29. ACM Digital Library. Web. 25 Nov. 2013.

---. "Storyspace 1." Proceedings of the Thirteenth ACM Conference on Hypertext and Hypermedia. New York, NY, USA: ACM, 2002. 172-181. ACM Digital Library. Web. 25 Nov. 2013. 
Bernstein, Mark, and Diane Greco, eds. Reading Hypertext. Watertown, MA: Eastgate Systems, 2009. Print.

Berry, R.M. "Metafiction" The Routledge Companion to Experimental Literature. Eds. Joe Bray, Alison Gibbons, Brian McHale. London; New York: Routledge, 2012. Print.

Birren, Faber. Color \& Human Response: Aspects of Light and Color Bearing on the Reactions of Living Things and the Welfare of Human Beings. New York: Van Nostrand Reinhold Co., 1978. Print.

Bolter, J. David. Writing Space: The Computer, Hypertext, and the History of Writing. L. Erlbaum Associates, 1991. Print.

Bolter, J. David. Getting Started with Storyspace for Macintosh. Watertown, MA: Eastgate Systems, 1994. Print.

Bolter, J. David, and Richard Grusin,. Remediation: Understanding New Media. Mit Press, 2000. Print.

Bolter, Jay David, and Michael Joyce. "Hypertext and Creative Writing." Proceedings of the ACM Conference on Hypertext. New York, NY, USA: ACM, 1987. 41-50. ACM Digital Library. Web. 25 Nov. 2013.

Bowie, David. Earthling Press Release. Virgin Records. 1995. TeenageWildLife.com. Web. 25 Nov 2013.

Bray, Joe, and Alison Gibbons, eds. Mark Z. Danielewski. Manchester; New York:

Manchester University Press, 2011. Print.

Bray, Joe, Alison Gibbons, and Brian McHale, eds. The Routledge Companion to Experimental Literature. London; New York: Routledge, 2012. Print.

Brick, Martin. "Blueprint (s): Rubric for a Deconstructed Age in House of Leaves." 2004. Sydney University Website. Web. 25. Nov. 2013.

Bruckheimer, Jerry et al. Black Hawk Down. Culver City, Calif.: Columbia TriStar Home Entertainment, 2002. Film.

Bury, Stephen. Artists' Books: The Book as a Work of Art, 1963-1995. Aldershot, Hants,

England; Brookfield, Vt., USA: Scolar Press, 1995. Print.

Ceruzzi, Paul E. A History of Modern Computing. MIT Press, 2003. Print.

---. "The Materiality of the Internet." IEEE Annals of the History of Computing 28.3 (2006): 96-96. IEEE Xplore. Web. 
Caramello, Charles. Silverless Mirrors: Book, Self \& Postmodern American Fiction. University Press of Florida, 1983. Print.

Chandler, Daniel. Semiotics: The Basics. Psychology Press, 2002. Print.

Ciccoricco, David. "Networked Narratives" The Routledge Companion to Experimental Literature. Eds. Joe Bray, Alison Gibbons, and Brian McHale. London; New York: Routledge, 2012. Print.

---. Reading Network Fiction. Tuscaloosa: University of Alabama Press, 2007. Print.

---. “The Materialities of Close Reading: 1942, 1959, 2009.” Digital Humanities Quarterly 6.1. (2012): n.pag. Web. 25 Nov. 2013.

Connor, Steven, ed. The Cambridge Companion to Postmodernism. Cambridge, UK; New York: Cambridge University Press, 2004. Print.

Coover, Robert. "Literary Hypertext: The Passing of the Golden Age." Keynote Address, Digital Arts and Culture .(1999): n.pag. Web. 25 Nov. 2013.

Cortázar, Julio. Hopscotch. New York: Pantheon Books, 1966. Print.

Currie, Mark. Metafiction. London: New York: Longman, 1995. Print.

Danielewski, Mark Z. House of Leaves. New York: Pantheon Books, 2000. Print.

---. The Fifty Year Sword. Amsterdam: De Bezige Bij, 2006. Print.

---. The Whalestoe Letters. New York: Pantheon Books, 2000. Print.

---. Live Q\&A on io9.com Mark Danielewski is here to answer your questions about the fifty year sword. (2012): n.pag. i09.com. Web. 25 Nov. 2013.

---. interview by Elina Shatkin. "Spooky Lit: Mark Z. Danielewski’s The Fifty Year Sword." LaMag.com 16 Oct. 2012. Web. 25 Nov 2013.

---. interview by Katherine Manderfield. "LAist Interview: Author Mark Z. Danielewski on 'The Fifty Year Sword,' the Written Word, and One of the Scariest Moments of His Life.” Laist.com 30 Oct. 2011. Web. 25 Nov 2013.

---. interview by Carolyn Kellogg. “A video interview with Mark Z. Danielewski.”

LaTimes.com. 31.10.2012. Web. 25 Nov 2013.

---. interview by Kasey Carpenter. "The Brash BoY, the MisunderstOod Girl and the Sonogram - the Books of Mark Z. Danielewski.” ChuckPalahniuk.net. 15 Sep. 2010. Web. 25 Nov. 2013.

---. interview by Stacey Knecht. The-ledge.com. 31 Oct. 2005. Web. 25 Nov. 2013. 
---. interview by Matthew Treon. "The Fifty Year Sword: An Interview With Mark Z.

Danielewski.” Litreactor.com. 16 Oct. 2012. Web. 25 Nov. 2013.

---. interview by Sophie Cottrell. “A conversation with Mark Danielewski."

RandomHouse.com. n.d. Web. 25 Nov. 2013.

---. interview by Eric Wittmershaus. "Profile: Mark Z. Danielewski."

TheModernWord.com. n.d. Web. 25 Nov. 2013.

Delany, Paul, and George Landow. Hypermedia and Literary Studies. London: The MIT Press, 1994. Print.

Douglas, J. Yellowlees. The End of Books—or Books Without End?: Reading Interactive Narratives. Ann Arbor: University of Michigan Press, 2000. Print.

Drucker, Johanna. "Humanities Approaches to Interface Theory.” Culture Machine 12 (2011): 1-20. Web. 25 Nov. 2013.

---. "Intimations of Immateriality." Reimagining Textuality: Textual Studies in the Late Age of Print. Eds. Elizabeth Bergmann Loizeaux and Neil Fraistat. University of Wisconsin Press, 2002. Print.

---. The Visible Word: Experimental Typography and Modern Art, 1909-1923. University of Chicago Press, 1997. Print.

Ensslin, Astrid. Canonizing Hypertext: Explorations and Constructions. New York: Continuum, 2007. Print.

Falco, Edward. Chemical Landscapes Digital Tales. Electronic Literature Collection Volume One, 2006. Web.

Faulkner, William. Absalom, Absalom! New York: Modern Library, 1951. Print. Foer, Jonathan Safran. Extremely Loud and Incredibly Close. Houghton Mifflin, 2006. Print. ---. Tree of Codes. Visual Editions Limited, 2010. Print.

Gass, William H. A Temple of Texts: Essays. New York: Alfred A. Knopf, 2006. Print.

---. Fiction and the Figures of Life. New York: Knopf, 1970. Print.

---. Finding a Form: Essays. New York: Knopf: Distributed by Random House, 1996. Print.

---. Habitations of the Word: Essays. New York: Simon and Schuster, 1985. Print.

---. Life Sentences: Literary Judgments and Accounts. New York: Alfred A. Knopf, 2012.

Print.

---. On Being Blue: a Philosophical Inquiry. Boston: D.R. Godine, 1975. Print.

---. Willie Masters' Lonesome Wife. [Evanston]: Northwestern University Press, 1968. Print. 
---. Willie Masters' Lonesome Wife. [Elmwood Park, IL]: Dalkey Archive Press, 1989. Print.

---. Willie Masters' Lonesome Wife. N.d. TS. Image Collections and Exhibitions at

Washington University in Saint Louis.

---. World Within The Word. Random House LLC, 2012. Print.

---. interview by Jan Garden Castro. “An Interview with William Gass.” Bulletin of the Association of Departments of English 70. 1981.30-34. Print

Gass, William H, and Theodore G. Ammon. Conversations with William H. Gass. Jackson: University Press of Mississippi, 2003. Print.

Gibbons, Alison. Multimodality, Cognition, and Experimental Literature. Routledge, 2012. Print.

Gibson, William, Dennis Ashbaugh, and Kevin Begos. Agrippa: A Book of the Dead. K. Begos, 1992. Print.

Glazier, Loss Pequeño. Digital Poetics: The Making of E-poetries. Tuscaloosa: University of Alabama Press, 2002. Print.

Gumbrecht, Hans Ulrich, and Karl Ludwig Pfeiffer, eds. Materialities of Communication. Stanford, Calif.: Stanford University Press, 1994. Print.

Hansen, Mark B. N. (Mark Boris Nicola). “The Digital Topography of Mark Z.

Danielewski’s House of Leaves.” Contemporary Literature 45.4 (2004): 597-636.

Project MUSE. Web. 25 Nov. 2013.

Harpold, Terry. "Harpold the contingencies of the hypertext link."Writing on the Edge, 2(1991): 126-139. News Media Reader. Web. 25 Nov. 2013.

---. Ex-foliations Reading Machines and the Upgrade Path. Minneapolis: University of Minnesota Press, 2009. Print.

Hayles, N. Katherine. Electronic Literature: New Horizons for the Literary. Notre Dame, Ind.: University of Notre Dame, 2008. Print.

---. How We Became Posthuman: Virtual Bodies in Cybernetics, Literature, and Informatics. Chicago, Ill.: University of Chicago Press, 1999. Print.

---. My Mother Was a Computer: Digital Subjects and Literary Texts. 2005. Print.

---. "Print Is Flat, Code Is Deep: The Importance of Media-Specific Analysis." Poetics

Today 25.1 (2004): 67-90. poeticstoday.dukejournals.org. Web. 13 Nov. 2013.

---. "Translating media: Why we should rethink textuality." The Yale Journal of Criticism 16.2 (2003): 263-290. Print. 
---. "Virtual Bodies and Flickering Signifiers." October 66 (1993): 69-91. JSTOR. Web. 25 Nov. 2013.

---. Writing Machines. Cambridge, Mass: Mediawork, 2002. Print.

Herman, David, ed. The Cambridge Companion to Narrative. Cambridge; New York:

Cambridge University Press, 2007. Print.

Herman, David, Manfred Jahn, and Marie-Laure Ryan, eds. Routledge Encyclopedia of

Narrative Theory. London; New York: Routledge, 2005. Print.

Holloway, Watson L. William Gass. Twayne, 1990. Print.

Hunt, John Dixon et al. Art, Word and Image: Two Thousand Years of Visual/textual

Interaction. London: Reaktion, 2010. Print.

Hustwit, Gary et al. Helvetica. [London]; Brooklyn, NY: Swiss Dots Ltd. ; Plexifilm :

Distributed by New video, 2007. Film.

Hutcheon, Linda. A Poetics of Postmodernism: History, Theory, Fiction. New York:

Routledge, 1988. Print.

---. Narcissistic Narrative: The Metafictional Paradox. Waterloo, Ont.: Wilfrid Laurier University Press, 1980. Print.

Joyce, Michael. Afternoon; a story. Watertown, MA: Eastgate Systems, 1990. Floppy Disc.

---. Afternoon; a story. Watertown, MA: Eastgate Systems, 2001. Compact Disc.

---. Moral Tales and Meditations: Technological Parables and Refractions. Albany: State

University of New York Press, 2001. Print.

---. Of Two Minds: Hypertext Pedagogy and Poetics. Ann Arbor: University of Michigan

Press, 1995. Print.

---. Othermindedness: The Emergence of Network Culture. Ann Arbor: University of

Michigan Press, 2000. Print.

---. The War Outside Ireland: a Novel. Jackson, Mich.: Tinkers Dam Press, 1982. Print.

---. Twelve Blue. . Watertown, MA: Eastgate Systems, 2004. Web.

Kaufmann, Michael. Textual Bodies: Modernism, Postmodernism, and Print. Bucknell

University Press, 1994. Print.

Kirschenbaum, Matthew G. Mechanisms: New Media and the Forensic Imagination.

Cambridge, Mass.: MIT Press, 2008. Print.

---. "Materiality and matter and stuff: what electronic texts are made of." Electronic Book

Review 3 (2001). Web. 25 Nov. 2013. 
Landow, George P. Hypertext: The Convergence of Contemporary Critical Theory and Technology. Baltimore: Johns Hopkins University Press, 1992. Print.

LeClair, Tom, and Larry McCaffery. Anything Can Happen: Interviews with Contemporary American Novelists. University of Illinois Press, 1983. Print.

Levy, Lawrence. Personal Interview. 11 Feb. 2011.

Loizeaux, Elizabeth Bergmann, and Neil Fraistat. Reimagining Textuality: Textual Studies in the Late Age of Print. Univ of Wisconsin Press, 2002. Print.

Mallarmé, Stéphane. A Throw of the Dice Never Will Abolish Chance. Limited Editions Club, 1993. Print.

Manovich, Lev. The Language of New Media. Cambridge, Mass.: MIT Press, 2002. Print. McCaffery, Larry. The Metafictional Muse: The Works of Robert Coover, Donald Barthelme, and William H. Gass. Pittsburgh, Pa.: University of Pittsburgh Press, 1982. Print.

McCaffery, Larry, and Sinda Gregory. "Haunted House-An Interview with Mark Z. Danielewski." Critique: Studies in Contemporary Fiction 44.2. 2003. 99-135. Print McDaid, John. Uncle Buddy's Phantom Funhouse. Watertown, MA: Eastgate Systems, 1992. Floppy Disc.

McHale, Brian. Postmodernist Fiction. New York: Methuen, 1987. Print.

Mitchell, W. J. T. Picture Theory: Essays on Verbal and Visual Representation. Chicago: University of Chicago Press, 1994. Print.

Montgomery, Lee B, and Mary Hussmann, eds. Transgressions: The Iowa Anthology of Innovative Fiction. Iowa City: University of Iowa Press, 1994. Print.

Moulthrop, Stuart. Hegirascope. Virginia Tech University. 1997. Web.

---. "You say you want a revolution? Hypertext and the laws of media." Postmodern Culture 1.3, 1991. Print.

---. Victory Garden. . Watertown, MA: Eastgate Systems, 1991. Floppy Disk.

Nafzawi, Muhammad Ibn Muhammad, Translated by Sir Richard Burton. The Perfumed Garden of the Shaykh Nefwazi. Indo-European Publishing, 2010. Print.

Nelson, Jason. Dreamaphage. Electronic Literature Collection Volume One. 2006. Web. Neumann, Birgit, and Ansgar Nünning. "The Living Handbook of Narratology.” N. p., 13 Mar. 2014. Print.

Olsen, Lance. 10:01. Chiasmus Press, 2005. Print. 
Olsen, Lance, and Tim Guthrie 10:01. Electronic Literature Collection Volume One, 2006. Web.

---. Architectures of Possibility: After Innovative Writing. Bowie, MD: Guide Dog Books, 2012. Print.

Phelan, James. A Companion to Narrative Theory. Malden, MA: Blackwell Pub., 2005.

Poe. Haunted. New York, NY: FEI/Atlantic, 2001. Audio Recording.

Pressman, Jessica. "House of Leaves': Reading the networked novel (Mark Z.

Danielewski)." Studies in American Fiction 34.1 (2006): 107-128. Print.

Punday, Daniel. Narrative after Deconstruction. SUNY Press, 2003. Print.

Romero, George A et al. Dawn of the dead. Troy, MI: Distributed by Anchor Bay

Entertainment, 2004. Film.

Ryan, Marie-Laure. Avatars of Story. U of Minnesota Press, 2006. Print.

---. Cyberspace Textuality: Computer Technology and Literary Theory. Bloomington:

Indiana University Press, 1999. Print.

---. Narrative Across Media: The Languages of Storytelling. Lincoln: University of

Nebraska Press, 2004. Print.

---. Narrative as Virtual Reality: Immersion and Interactivity in Literature and Electronic

Media. Baltimore: Johns Hopkins University Press, 2001. Print.

---. Possible Worlds, Artificial Intelligence, and Narrative Theory. Bloomington: Indiana

University Press, 1991. Print.

Shiovitz, Dan. Bad Machine. Electronic Literature Collection Volume One, 2006. Web.

Shlain, Leonard. The Alphabet Versus the Goddess: The Conflict Between Word and Image. Penguin Group US, 1990. Print.

Sloane, Sarah. Digital Fictions: Storytelling in a Material World. Greenwood Publishing Group, 2000. Print.

Smith, Sarah. King of Space. Watertown, MA: Eastgate Systems, 1991. Floppy Disc.

Sondheim, Alan. Internet Text. Electronic Literature Collection Volume One. 2006. Web.

Sterne, Laurence. The Life and Opinions of Tristram Shandy, Gentleman. Oxford:

Clarendon Press, 1983. Print.

Szilak, Illya. Reconstructing Mayakovsky. Electronic Literature Collection Volume Two. 2011. Web.

Tabbi, Joseph. Cognitive Fictions. University of Minnesota Press, 2002. Print. 
Tanner, Tony. Scenes of Nature, Signs of Men. Cambridge; New York: Cambridge University Press, 1987. Print.

Ulmer, Gregory L. Internet Invention: From Literacy to Electracy. Longman, 2003. Print. Walker, Jill. "Piecing Together and Tearing Apart: Finding the Story in afternoon." Reading Hypertext. Eds. Mark Bernstein and Diane Greco. 21-34. Watertown, MA: Eastgate Systems, 2009. Print.

Wardrip-Fruin, Noah, and Pat Harrigan. First Person: New Media as Story, Performance, and Game. Cambridge, Mass.: MIT Press, 2004. Print.

Waugh, Patricia. Metafiction: The Theory and Practice of Self-conscious Fiction. London; New York: Methuen, 1984. Print.

Wenderoth, Joe. Letters to Wendy's. Athens, GA: Verse Press, 2000. Print.

White, Glyn. "Reading the graphic surface of Mark Z. Danielewski's The Fifty Year Sword." Mark Z. Danielewski. Eds. Joe Bray, Alison Gibbons and Brian McHale. Manchester; New York: Manchester University Press, 2011. Print.

---. Reading the Graphic Surface: The Presence of the Book in Prose Fiction. Manchester, UK; New York : New York: Manchester University Press, 2005. Print.

Wilheim, Jorge. Fax, Messages from a Near Future. London: Earthscan Publications, 1994. Print. 